\title{
The role of complexin I in synaptic transmission at the mouse calyx of Held synapse
}

\author{
Dissertation \\ for the award of the degree \\ "Doctor rerum naturalium" \\ Division of Mathematics and Natural Sciences \\ of the Georg-August-Universität Göttingen \\ within the doctoral program (Sensory and Motor Neuroscience) \\ of the Georg-August University School of Science (GAUSS)
}

\section{Submitted by \\ Shuwen Chang}

From Pingtung, Taiwan

Göttingen 2013 


\section{Declaration}

I hereby declare that my $\mathrm{PhD}$ thesis 'The role of complexin I in synaptic transmission at the mouse calyx of Held synapse' has been written independently with no other aids or sources than quoted.

Göttingen, July 31th 2013 


\section{Contents}

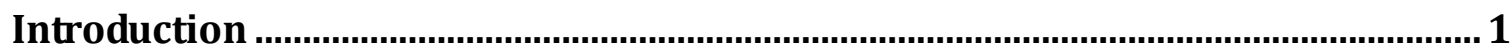

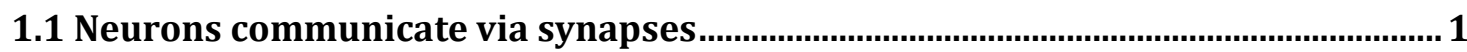

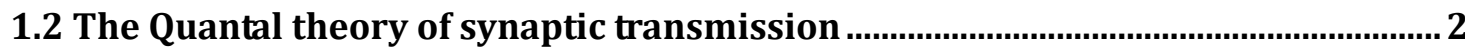

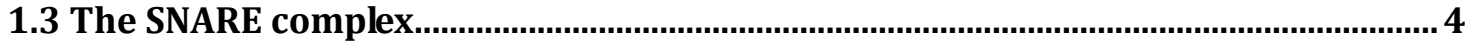

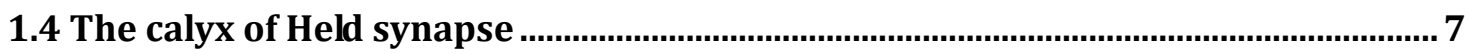

1.5 Developmental changes at calyx of Held during maturation ....................................... 9

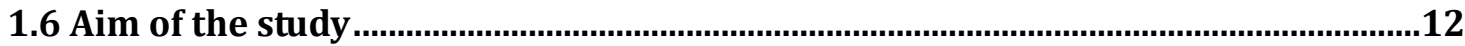

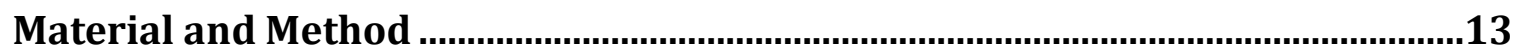

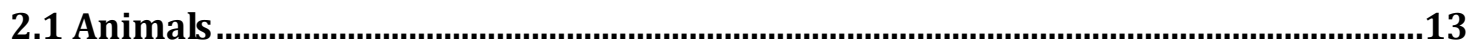

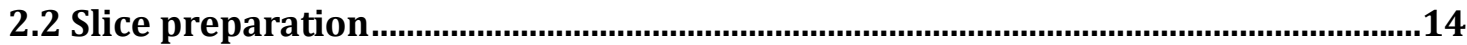

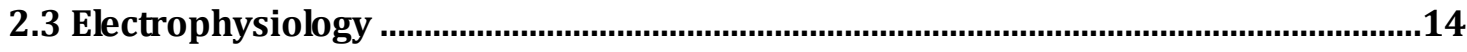

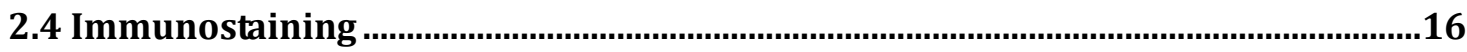

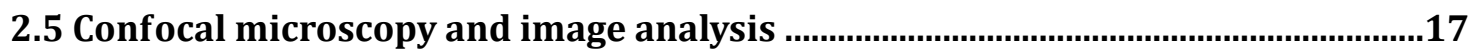

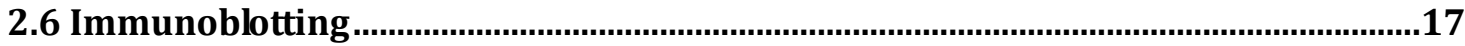

2.7 Reverse transcription and real-time PCR ................................................................

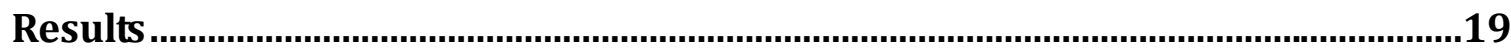

3.1 Quantitative analysis of CPX expression in the MNTB region. ...................................19

3.1.1 CPXI is the predominant complexin isoform at calyx of Held ....................................... 19

3.1.2 CPXI is pre- and postsynaptically expressed in the MNTB region............................... 22

3.2 Evaluation of synaptic strength in CPXI-deficient calyx synapses .........................23

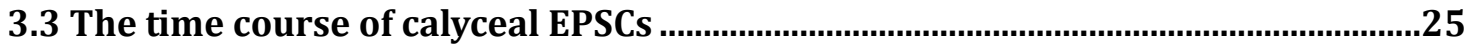

3.4 Mechanisms that underlie the reduced synaptic strength in $\mathrm{CPXI} /$ - calyces..........27

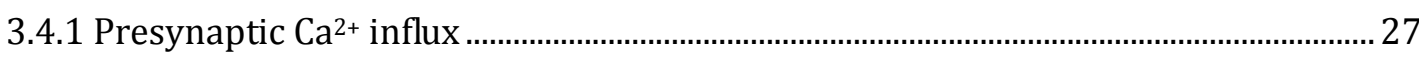

3.4.2 The size of the readily releasable pool (RRP) .................................................................. 28

3.4.3 Comparison of mEPSC amplitudes, kinetics and frequency in $\mathrm{CPX} \%$ and wt

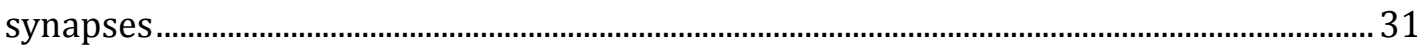

3.4.4 Comparison of time course of synchronous release transients..................................... 34

3.4.5 Calyceal action potential waveform and $\mathrm{Ca}^{2+}$ channel coupling....................................36

3.5 Comparison of short-term plasticity in $\mathrm{CPX} \%$ and wt synapses.................................39

3.6 Rescue of altered EPSC amplitudes and short-term plasticity in CPX $/$ -

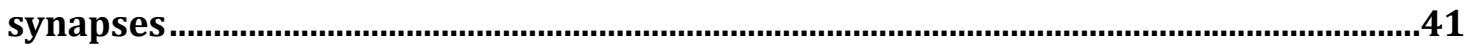

3.7 Strongly enhanced asynchronous release in mature CPXI-/-synapses......................42

3.8 Attenuating presynaptic residual $\mathrm{Ca}^{2+}$ suppressed asynchronous vesicles

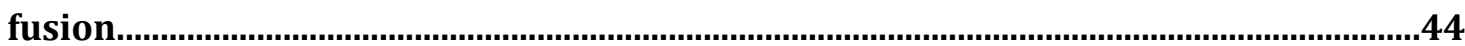

3.9 Asynchronous release following EPSC trains in $\mathrm{CPXI}^{-/-}$synapses is $\mathrm{Ca}^{2+}$ dependent 
3.10 Correlation between asynchronous release and synchronous release.................47

3.11 Aberrant postsynaptic AP firing in CPXI $/$ - synapses..............................................49

3.12 Delayed recovery of EPSCs from depression in CPXI $/$ synapses ...........................51

3.13 Blocking asynchronous release in $\mathrm{CPXI} /$ - terminals augments subsequent

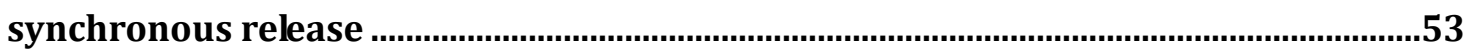

3.14 Down regulation of CPXII at calyx of Held synapses during development ...........55

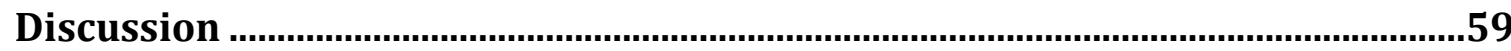

4.1 Multiple roles of CPXI in regulating vesicle exocytosis .......................................60

4.2 More than one mechanism for neurotransmitter release .......................................62

4.3 A possible defect in the coupling between VGCCs and docked vesicles.....................64

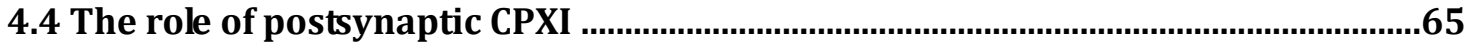

Summary

Appendix

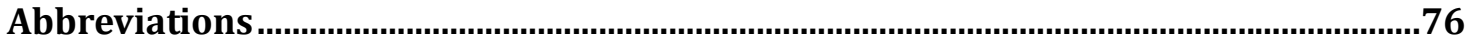

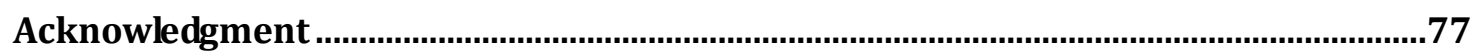

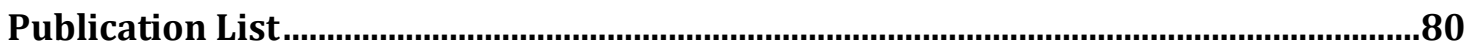




\section{Introduction}

\subsection{Neurons communicate via synapses}

Synaptic transmission is the key process for information exchange and communication between individual neurons. It involves presynaptic $\mathrm{Ca}^{2+}$ influx triggering exocytosis of synaptic vesicles at the presynaptic active zone of nerve terminals and leads to the activation of postsynaptic receptors by the transmitter molecules liberated into the synaptic cleft. Fast synaptic transmission is initiated when an action potential (AP) invades a nerve terminal and opens voltage-gated $\mathrm{Ca}^{2+}$ channels. This results in an intracellular $\mathrm{Ca}^{2+}\left(\left[\mathrm{Ca}^{2+}\right]_{\mathrm{i}}\right)$ transient which triggers neurotransmitter release within a millisecond or less. Although vesicle fusion happens primarily in response to stimulation, it can also occur spontaneously. In order to quickly respond to the presynaptic $\mathrm{Ca}^{2+}$ rise, synaptic vesicles which are ready to be released are docked onto specialized sites of the plasma membrane called active zones. A priming reactions arrests the vesicles in a metastable state that is extremely sensitive to any entry of $\mathrm{Ca}^{2+}$ (Rizo and Rosenmund, 2008). Thus, fluctuations of $\left[\mathrm{Ca}^{2+}\right]_{\mathrm{i}}$ might occasionally trigger the spontaneous fusion of vesicles (Angleson and Betz, 2001; Dittman and Regehr, 1996). These events give rise to miniature postsynaptic potentials (mPSPs) in postsynaptic cells (Katz, 1969). It is believed that the distance of a vesicle to the $\mathrm{Ca}^{2+}$ channels (30 to $300 \mathrm{~nm}$ ) critically determines the release probability (from $<0.01$ to 1 ) (Sakaba and Neher, 2001). Through sophisticated $\mathrm{Ca}^{2+}$ uncaging experiments at the calyx of Held, the approximate $\mathrm{Ca}^{2+}$ concentration ranges and cooperativity for synchronous, asynchronous and spontaneous release have been established (Bollmann et al., 2000; Kochubey et al., 2011; Schneggenburger and 
Neher, 2000). However, whether the intricate presynaptic $\mathrm{Ca}^{2+}$ signal alone is sufficient to generate the complexity in neurotransmitter release is still in debate. For example, Groffen et al., (2010) proposed that different release modes are triggered by separate $\mathrm{Ca}^{2+}$ sensors with distinct kinetics and possibly different $\mathrm{Ca}^{2+}$ cooperativities. At present, not only many details of the vesicle release machinery, but also the origins of functionally distinct vesicle pools (Sara et al., 2005) and the precise mechanisms generating divergent release modes are still incompletely understood.

\subsection{The Quantal theory of synaptic transmission}

Early work by Katz and collaborators (Katz, 1969) on the frog neuromuscular junction showed that transmitter is released in discrete packages. A single transmitter package (quantum) gives rise to a miniature postsynaptic current or potential. After presynaptic AP arrival, numerous quanta are released in parallel. Thus, the amplitude of postsynaptic current (PSC) is determined by the product of the postsynaptic quantal size $(q)$, the number of presynaptic release sites $(N)$, and the release probability of vesicle $(p)$, i.e PSC $=N \times p \times q$. The quantal size $q$ is defined as the current generated by a miniature postsynaptic current and $N$ is the number of vesicles immediately available for release. This concept has been an important and practical framework for several decades of research, because it allows a separation of the contributions from the presynaptic and the postsynaptic side to the regulation of synaptic transmission (Quastel, 1997; Scheuss and Neher, 2001; Schneggenburger et al., 2002). If $N$ is regarded as the number of vesicles in the readily releasable pool, then $p$ will refer to the probability that a vesicle is released from that pool upon AP arrival (Schneggenburger, Sakaba et al. 2002). Most synapses rely on three vesicle pools: the readily releasable pool, the recycling pool and the reserve pool. The readily releasable 
pool is a population of vesicles which are very close to the presynaptic active zone, and usually undergo exocytosis immediately after stimulation. It generally consists of no more than $\sim 1-2 \%$ of the total vesicles. The reserve pool, which is relatively immobile compared to the other pools, comprises the majority of total vesicles $(\sim 80-90 \%)$. Finally, the newly recycled vesicles which are refilled right after endocytosis to replenish the readily releasable pool, account for $5-20 \%$ of the total vesicles (Rizzoli and Betz, 2005). The mechanisms that sort vesicles into distinct pools and enable certain vesicles to participate in a specific physiological activity remain largely obscure.

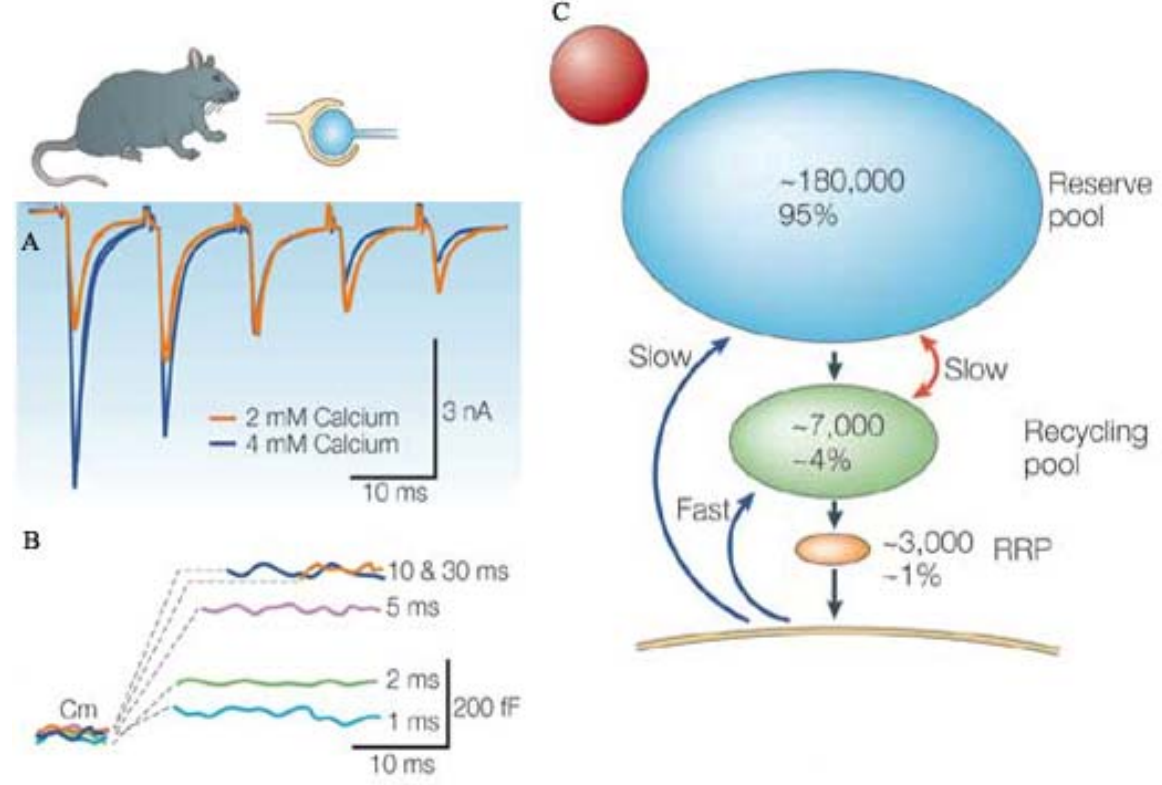

The vesicle pools at calyx of Held synapses: (a)(b) demonstrated two methods which are used for estimating the readily releasable pool at calyx of Held synapses (a) Postsynaptic current recording under $100 \mathrm{~Hz}$ stimulation; the RRP is rapidly depleted. (b) Presynaptic capacitance response to short (1-30 ms) depolarization; the response (indicative of readily releasable pool) plateaus at the step of $10 \mathrm{~ms}$ of depolarization. $\left(\mathrm{C}_{\mathrm{m}}\right.$, membrane capacitance) (c) Pool sizes and mixing pathways. Blue arrows indicate endocytosis; red arrows indicate mixing between pools. The red circle 
indicates the total pool size relative to other preparations. Panels were reproduced with permission of Nature Reviews Neuroscience. (Rizzoli and Betz, 2005)

\subsection{The SNARE complex}

Vesicle exocytosis is triggered by presynaptic $\mathrm{Ca}^{2+}$ influx and happens within a millisecond or less after AP arrival. It requires assembly of a protein complex termed SNARE (soluble N-ethylmaleimide-sensitive factor attachment protein receptor) complex, composed of plasma membrane proteins, syntaxin-1 and SNAP-25, and of the vesicle protein synaptobrevin/VAMP (Rizo and Rosenmund, 2008; Söllner et al., 1993; Sudhof and Rothman, 2009). Formation of this complex overcomes the strong electrostatic repulsion between the plasma membrane and the vesicle membrane by forming a parallel four-helical bundle consisting of one SNARE motif from Synataxin, two helices from SNAP-25, and one helix from synaptobrevin/VAMP embedded in the vesicle membrane. However, the fusion event is restrained until an AP propagates into the terminal and elicits a $\left[\mathrm{Ca}^{2+}\right]_{\mathrm{i}}$ transient. After the SNARE complex accommodates with the $\mathrm{Ca}^{2+}$ sensor synaptotagmin, it will complete its full assembly, and fuel membrane fusion. In order to execute fusion efficiently, four families of small proteins are involved in the regulation of SNARE complexes, which include Munc18 and Munc13 that prime vesicle for assembling, and synaptotagmin and complexin that take control of $\mathrm{Ca}^{2+}$ signaling (Jahn and Fasshauer, 2012).

Synaptotagmin is a prominent $\mathrm{Ca}^{2+}$ sensor for fast synchronous release, binding to $\mathrm{Ca}^{2+}$ through two $\mathrm{C} 2$ domains (known as $\mathrm{C} 2 \mathrm{~A}$ and $\mathrm{C} 2 \mathrm{~B}$ ). In mammals, there are 15 Syt isoforms, and Syt-1 and Syt-2 are predominantly expressed in the brain (Sudhof, 2002; Xiao et al., 2010). Deletion of Syt-1 and Syt-2 led to a strong reduction of synchronous release, but spontaneous release remained intact, suggesting that 
additional proteins might exist which regulate slow asynchronous release and spontaneous release (Kochubey et al., 2011; Sun et al., 2007). Using a Syt-2 mutant that contains a single aspartate mutation in the $\mathrm{C} 2 \mathrm{~B}$ domain (D364N) of synaptotagmin-2, Kochubey and Schneggenburger (2011) showed that the release clamping function was partially mediated by the poly-lysine motif of the $\mathrm{C} 2 \mathrm{~B}$ domain.

Complexins are relatively small synaptic proteins, which were first described in 1995, that functionally cooperate with synaptotagmin. They are encoded by the Cplx $1-4$ genes which drive the expression of the four isoforms CPXI IV in mammals (McMahon et al., 1995; Reim et al., 2001). Complexins are soluble proteins. They are not only expressed in presynaptic terminals but also found postsynaptically (Reim et al., 2005). In mammals, CPXI is exclusively expressed in the central nervous system, where its mRNA expression pattern overlapped with that of complexin II in many brain regions (Freeman and Jennifer Morton, 2004; McMahon et al., 1995; Reim et al., 2005). Divergent models for their function have been proposed, but it is generally agreed that complexin's function is to prime vesicles and activate synchronous release (Reim et al., 2001; Xue et al., 2007) and to clamp vesicles from being spontaneously released (Kaeser-Woo et al., 2012; Tang et al., 2006). However, contrasting effects on spontaneous release were observed in different $\mathrm{KO} / \mathrm{KD}$ experiments when studying CPXI's function: Knocking down complexin expression by RNA interference in cortical culture neurons increased spontaneous vesicle fusion events (Maximov et al., 2009), but hippocampus autaptic knockout neurons showed the opposite (Xue et al., 2007). Thus, a clamping function of complexin is still debated. The fusion clamp function of complexin was first postulated by Giraudo et al. (2006), who demonstrated that complexin can freeze the SNAREpin, an assembled intermediate 
state of SNARE complex, and release the block in the presence of $\mathrm{Ca}^{2+}$ and synaptotagmin. The following year, Littleton's lab demonstrated that complexin regulates spontaneous release by preventing premature vesicle exocytosis in Drosophila melanogaster complexin null mutants (Huntwork and Littleton, 2007). In subsequent years, the multiple faces of complexin have been unveiled piece by piece through sophisticated molecular domain function studies. There are four functional domains within complexin: the $\mathrm{N}$-terminus is required for activating fusion (Maximov et al., 2009; Xue et al., 2007), the accessory $\alpha$-helix exhibits an inhibitory effect by restricting spontaneous release (Maximov et al., 2009), the central $\alpha$-helix of complexin is essential for SNARE complex binding (Tang et al., 2006), and the C-terminus is selectively required for clamping and priming (Kaeser-Woo et al., 2012; Martin et al., 2011; Xue et al., 2007). To explain its dual stimulatory and inhibitory roles, two mechanisms have been postulated. First, the binding of complexin to the SNARE complex promotes the initiation of the assembling process and stabilizes the half-zippered complex. Second, it blocks progression of SNARE-zippering via its accessory $\alpha$-helix domain, the sequence which competes directly with VAMP to bind to SNARE, and this clamp can eventually be released upon $\mathrm{Ca}^{2+}$ triggering (Jahn and Fasshauer, 2012). On the other hand, genetic deletion of complexin showed a similar phenotype to synaptotagmin knock-out mice, suggesting that both proteins might exert a similar function. 


\subsection{The calyx of Held synapse}

The calyx of Held is a large synapse in the mammalian auditory brainstem that is part of the circuitry underlying sound-source localization. The accessibility of its presynaptic terminal for patch pipettes has enabled direct studies of presynaptic aspects of synaptic transmission (Borst et al., 1995; Forsythe, 1994; Kuwabara et al., 1991). The calyx synapse was first described by the German anatomist Hans Held in 1893, who used the newly developed Gogli staining method in the cat auditory brainstem (Borst and Soria van Hoeve, 2012). Held showed that the afferent axons sent by globular bushy cells in the Ventral Cochlear Nucleus (VCN) eventually form calyx-like terminals on to the principal cells in the contralateral Medial Nucleus of the Trapezoid body (MNTB) (von Gersdorff and Borst, 2002). Before hearing onset, a single presynaptic calyx can contact approximately two thirds of the principal cell surface. The vast majority of MNTB principal neuron receives one calyx synapse, and only $\sim 5 \%$ have multiple inputs (Bergsman et al., 2004; Rodriguez-Contreras et al., 2008). Because of its accessibility to patch-clamp recordings, the calyx of Held synapse has become a model system for studying presynaptic function. Direct recordings from the calyx of Held presynaptic terminal were first achieved in 1994 (Forsythe, 1994). Subsequently, many presynaptic functional properties such as ion

channel properties, $\mathrm{Ca}^{2+}$ dependent vesicle exocytosis, and short-term plasticity have been studied (Schneggenburger and Forsythe, 2006).

The calyx of Held receives auditory information from the contralateral globular bushy cell axons. Globular bushy cells are glutamatergic neurons. Owing to the thick, myelinated axons of the globular busy cells, the minimum sound response latency of principal neurons is only 3 to $5 \mathrm{~ms}$. During tone presentations, calyx synapses rarely fail. Thus, they can transmit up to $\sim 300 \mathrm{~Hz}$ without attenuation (Borst and Soria van 
Hoeve, 2012). Due to its big size, the calyx can accommodate approximately 300-700 active zones and can release more than 100 vesicles in response to a single AP (Satzler et al., 2002). In the past decades, the mechanisms underlying synaptic transmission and plasticity at calyx of Held synapses have been extensively studied (Borst and Sakmann, 1999; Iwasaki et al., 2000; Taschenberger et al., 2002; Taschenberger and von Gersdorff, 2000). Similar to many other synapses in the brain, synaptic strength at the calyx of Held is regulated by a variety of either short-lived or long-lasting processes, some of which lead to a decrease in synaptic strength and others lead to synaptic enhancement (Zucker and Regehr, 2002). Mechanisms that regulate synaptic strength on a relatively short time scale of tens of ms to several minutes generate short-term plasticity. At calyx of Held synapses, several mechanisms that contribute to short-term plasticity have been identified, including depletion of synaptic vesicles, $\mathrm{Ca}^{2+}$-dependent acceleration of vesicle replenishment, $\mathrm{Ca}^{2+}$ dependent facilitation of release, activation/inactivation of presynaptic $\mathrm{Ca}^{2+}$ channels, and saturation/desensitization of postsynaptic receptors. The relative contribution of the different mechanisms depends on stimulus pattern and developmental stage (Borst and Soria van Hoeve, 2012).

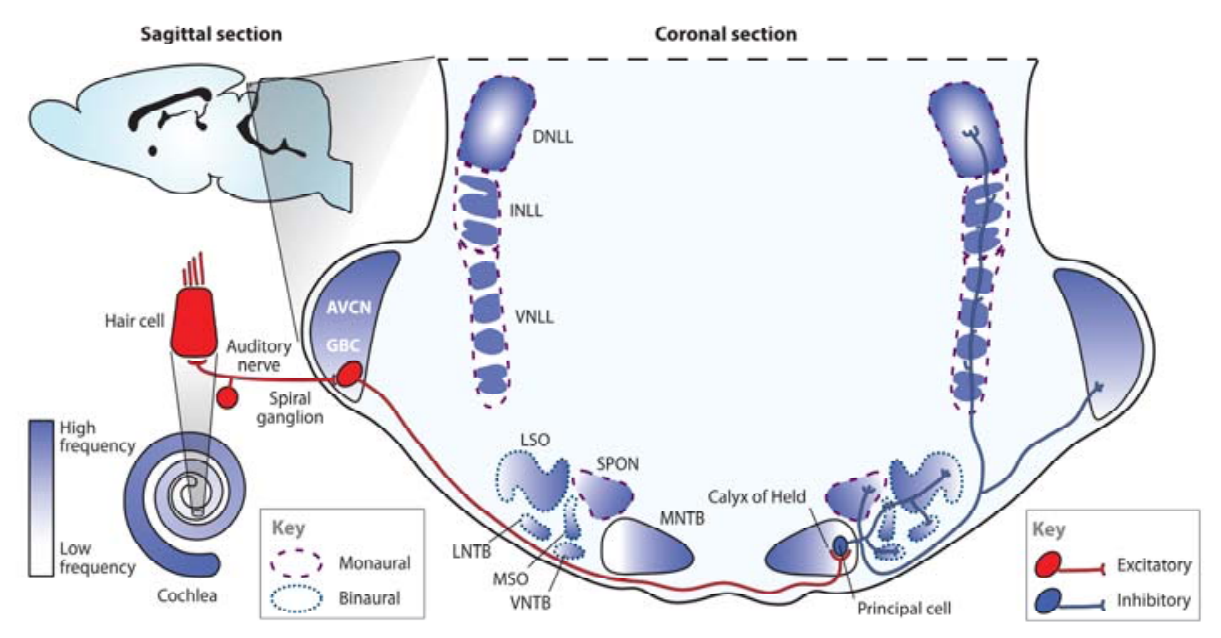


Anatomical connection of principal neurons of MNTB region. Principal neurons in the medial nucleus of the trapezoid body (MNTB) receive a single large calyceal input (red), which originates from globular bushy cells (GBC) in the anteroventral cochlear nucleus (AVCN). The GBC receive direct inputs from the auditory nerve. Image was reproduced with permission of Annual Reviews of Physiology. (Borst and Soria van Hoeve, 2012)

\subsection{Developmental changes at calyx of Held during maturation}

During postnatal maturation, the calyx of Held synapse undergoes miscellaneous morphological and functional transformations that collectively aid in establishing signal transduction at high speed and fidelity. Morphologically, the calyx terminal changes from a spoon-like to a multidigit-like structure. This morphological change is accompanied by several physiological changes, including briefer AP waveform with an average halfwidth of $\sim 0.4 \mathrm{~ms}$ at P8-10 and $\sim 0.27 \mathrm{~ms}$ at P16 (Yang and Wang, 2006), as well as a tighter spatial coupling between voltage-gated $\mathrm{Ca}^{2+}$ channels (VGCCs) and synaptic vesicles. The tighter topological arrangement of docked vesicles relative to VGCCs allows vesicles being exposed to higher intracellular $\mathrm{Ca}^{2+}$ concentration, and hence guaranties high quantal output in mature calyces (Wang et al., 2008). In addition, a developmental increase in the number of active zones and the size of the pool of readily releasable synaptic vesicles has been demonstrated (Taschenberger et al., 2002). Finally, the quantal size estimated by mEPSC recordings also increased from an average of 35-40 pA (P5-7) to 55-60 pA in older rats (P12-14) (Taschenberger et al., 2005) and the decay kinetics of AMPAR- and NMDAR-EPSCs accelerates in mature synapses. (Joshi and Wang) demonstrated that the time constants of AMPAR-EPSCs decreased by about $70 \%$ from P5 to P13. Rapid kinetics of AMPAR-mediated EPSCs is thought to be an adaptation and prerequisite for the preservation of timing information in auditory circuits (Trussell, 1999). Taken together, these changes shorten the synaptic delay, prevent the premature depletion of 
vesicles, and reduce the desensitization of postsynaptic receptors, and therefore attenuate the amount of synaptic depression and allows high-frequency transmission (von Gersdorff and Borst, 2002). The major developmental changes at the rodent calyx of Held synapse have been summarized in (Borst and Soria van Hoeve, 2012) as shown below:

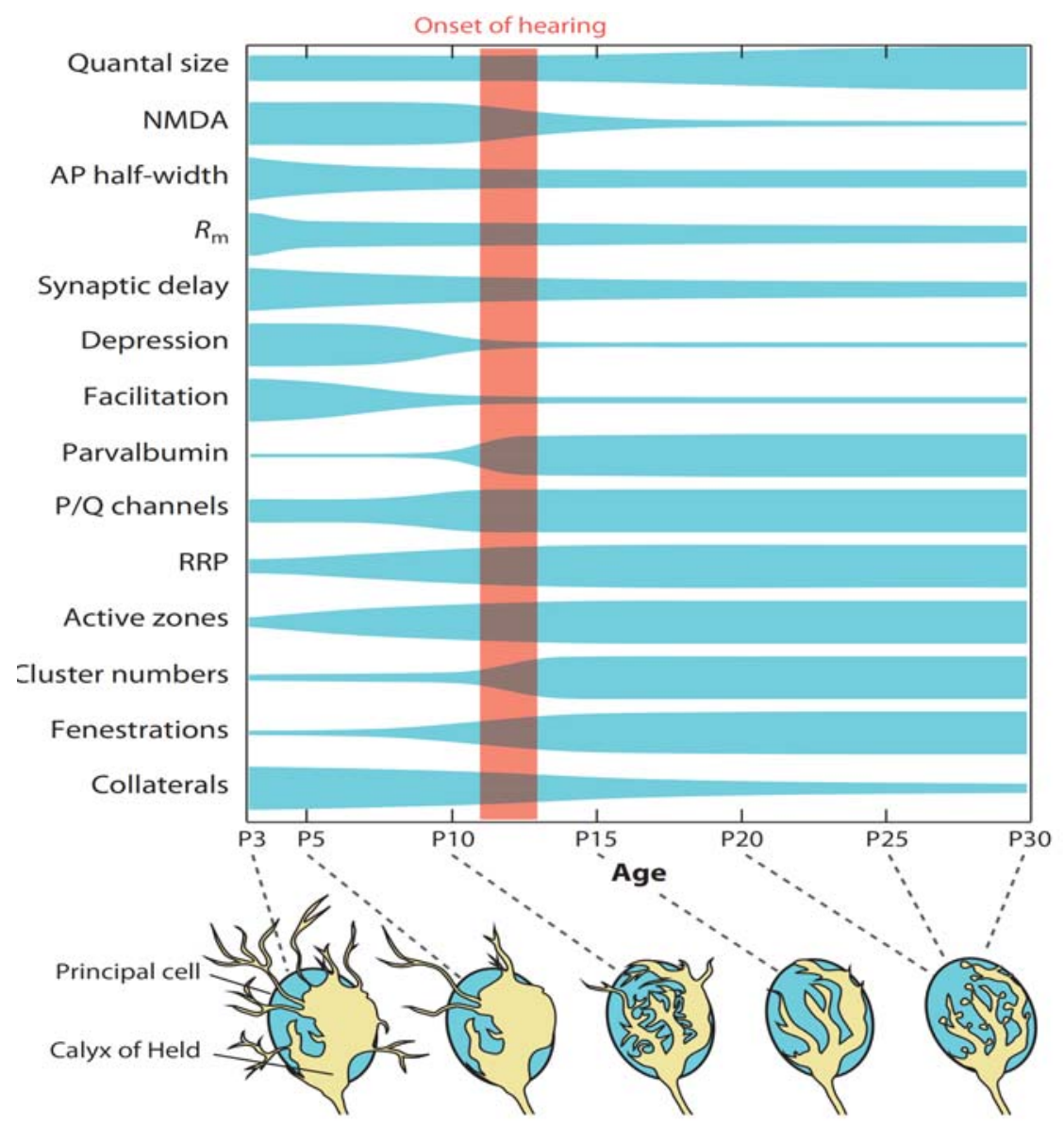

Timeline of major developmental steps in the rodent calyx of Held synapse. (1) quantal size, miniature excitatory postsynaptic current (EPSC) amplitude (2) NMDA, size of synaptic NMDA-type glutamate receptor conductance (3) AP half-width, full width at half-maximum of the postsynaptic action potential during slice whole-cell recordings or estimated on the basis of juxtacellular in vivo recordings (4) $R_{\mathrm{m}}$, postsynaptic membrane resistance (5) synaptic delay, delay between in vivo pre- and postsynaptic action potentials (6) depression, amount of short-term depression during in vivo spontaneous activity [this measure is analogous to measurements of release probability in slices] (7) facilitation, short-term facilitation decay time constant (8) parvalbumin, presynaptic parvalbumin concentration [a somewhat earlier onset has also been reported] (9) P/Q channels, fraction of presynaptic calcium channels that are $\mathrm{P} / \mathrm{Q}$ type (10) RRP, size of the readily releasable pool [for the earliest time points only the size of the EPSC has been measured] (11) Active zones denotes number of active zones (12) cluster numbers, number of vesicle clusters (13) fenestrations, appearance of fenestrations in the calyx of Held (14) collaterals, total length of calycine collaterals; 
age, age in postnatal days (P) Image was reproduced with permission of Annual Reviews of Physiology. (Borst and Soria van Hoeve, 2012) 


\subsection{Aim of the study}

Complexins are essential synaptic proteins that are involved in regulating synaptic transmission. Perturbation of complexin expression or genetic ablation revealed both facilitatory and inhibitory roles of this protein in regulating SNARE-mediated vesicle fusion. However, many of these results were obtained by using either model organisms such as Drosophila melanogaster or in neuronal cultures whereas relatively little is known about complexin's function in native mammalian synapses. Direct presynaptic recordings are difficult and remain limited to a small number of presynaptic terminals that are large enough to be accessible by patch electrodes. Here, we study the function of complexin I (CPXI) at the mouse calyx of Held synapse, a large synapse in the mammalian auditory brainstem, which enables us to directly measure presynaptic functional parameters. Because complexin I knock-out mice are viable, we are able to study complexin's function over a wide range of developmental stages of the calyx synapse. 


\section{Material and Method}

\subsection{Animals}

The generation of CPXI knock-out mice has been described previously (Reim et al., 2001). CPXI deficient mice were backcrossed for at least five generations into the C57BL/6N genetic background. For genotyping PCR analysis, 1-2 mm tissue sections of the mouse tail tip were dissolved in $0.5 \mathrm{ml}$ of $100 \mathrm{mM}$ Tris ( $\mathrm{pH} \mathrm{8.5),} 5 \mathrm{mM}$ EDTA, $0.2 \% \mathrm{SDS}, 0.2 \mathrm{M} \mathrm{NaCl}$ and $1 \mathrm{mg} / \mathrm{ml}$ proteinase $\mathrm{K}$ solution at $55^{\circ} \mathrm{C}$ for at least four hours with vigorous shaking. The extracts were centrifuged at 13,000 rpm for 5 minutes and $300 \mu \mathrm{l}$ of the supernatant was transferred to a fresh Eppendorf tube. This supernatant was mixed with $300 \mu \mathrm{l}$ isopropanol and left at room temperature for 2 minutes. Following centrifugation at 13,000 rpm for 5 minutes, the pellet was washed with $500 \mu 170 \%$ ethanol, then vacuum dried and finally dissolved in $60 \mu \mathrm{l}$ distilled water. This DNA is stable at $4^{\circ} \mathrm{C}$. PCR was then performed with primers $1118 / 1119$ for wt mice and 1111/1112 for $\mathrm{CPXI}^{-/-}$mice sequences $\left(1118=5^{\prime}\right.$-AGT ACT TTT GAA TCC CCT GGT GA-3'; 1119=5'-TAG CTA TCC CTT CTT GTC CTT GTG-3';1111=5'-CGC GGC GGA GTT GTT GAC CTC G-3';1112=5'-CTG GCT TGT CCC TGA ATC CTG TCC-3') with PhireHotStart Polymerase (Finnzymes \#F-120S) using the following conditions: $94^{\circ} \mathrm{C}$ for $30 \mathrm{~s}, 64^{\circ} \mathrm{C}$ for $30 \mathrm{~s}, 72^{\circ} \mathrm{C}$ for $60 \mathrm{~s}$, for 32 cycles. One half of the PCR reaction was then analyzed on a 1.5\% agarose gel and visualized by ethidium bromide staining. Homozygous $\mathrm{CPXI}^{+/+}, \mathrm{CPXI}^{-/-}$and heterozygous $\mathrm{CPXI}^{+/-}$littermates as well as $\mathrm{C} 57 \mathrm{BL} / 6 \mathrm{~N}$ mice at the age of postnatal days (P) 8 to 26 were included in this study. All experiments complied with national animal care guidelines. 


\subsection{Slice preparation}

Homozygous, $\mathrm{CPXI}^{+/+}$and $\mathrm{CPXI}^{-/-}$littermates of either sex were used for most of the experiments. For comparison, some recordings were also obtained from C57BL/6N mice. Brainstem slices were prepared as described previously (Taschenberger and von Gersdorff, 2000). After decapitation, the whole brain was immediately immersed into ice-cold low $\mathrm{Ca}^{2+}$ artificial CSF (aCSF) containing the following (in $\mathrm{mM}$ ): $125 \mathrm{NaCl}$, $2.5 \mathrm{KCl}, 3 \mathrm{MgCl}_{2}, 0.1 \mathrm{CaCl}_{2}, 10$ glucose, $25 \mathrm{NaHCO}_{3}, 1.25 \mathrm{NaH}_{2} \mathrm{PO}_{4}, 0.4$ ascorbic acid, 3 myo-inositol, and 2 Na-pyruvate at $\mathrm{pH} 7.3$ when bubbled with carbogen $(95 \%$ $\mathrm{O}_{2}, 5 \% \mathrm{CO}_{2}$ ). The brainstem was glued onto the stage of a VT1000S vibratome (Leica), and $200 \mu \mathrm{m}$-thick coronal slices containing the MNTB were cut. Slices were incubated for $30-40 \mathrm{~min}$ at $35^{\circ} \mathrm{C}$ in a chamber containing normal aCSF and kept at room temperature $\left(21-24{ }^{\circ} \mathrm{C}\right)$ for up to $5 \mathrm{~h}$ thereafter. The composition of normal aCSF was identical to that of low $\mathrm{Ca}^{2+}$ aCSF, except that $3 \mathrm{mM} \mathrm{MgCl} 2$ and $0.1 \mathrm{mM}$ $\mathrm{CaCl}_{2}$ were replaced with $1 \mathrm{mM} \mathrm{MgCl}$ and $2 \mathrm{mM} \mathrm{CaCl}_{2}$.

\subsection{Electrophysiology}

Whole-cell patch-clamp recordings were made from calyx of Held terminals and principal cells using an EPC-10 amplifier controlled by "Pulse" or "PatchMaster" software (HEKA Elektronik, Germany). Sampling intervals and filter settings were 20 $\mu$ s and $5.0 \mathrm{kHz}$, respectively. Cells were visualized by infrared-differential interference contrast microscopy through $40 \times$ or $60 \times$ water-immersion objectives using an upright BX51WI microscope (Olympus, Germany). All experiments were carried out at room temperature $\left(21-24{ }^{\circ} \mathrm{C}\right)$. All values are given as mean $\pm \mathrm{SEM}$. Unless indicated otherwise, significance of difference was evaluated with the two-tailed Student's unpaired $t$ test and $p<0.05$ was taken as the level of statistical significance. 


\section{Presynaptic recordings:}

Patch pipettes were pulled from borosilicate glass (Science Products) on a P-87 micropipette puller (Sutter Instrument, USA). Pipettes were coated with dental wax to reduce stray capacitance. Open tip resistance was 4-5 $\mathrm{M} \Omega$. Access resistance $\left(R_{\mathrm{S}}\right)$ was $\leq 20 \mathrm{M} \Omega$ and routinely compensated by $50-60 \%$ during presynaptic voltage-clamp experiments. The holding potential $\left(V_{\mathrm{h}}\right)$ was $-80 \mathrm{mV}$. For measuring presynaptic $I_{\mathrm{Ca}(\mathrm{V})}$ and changes in membrane capacitance $\left(\Delta C_{\mathrm{m}}\right)$, pipettes were filled with a solution containing the following (in $\mathrm{mM}$ ): 140 Cs-gluconate, 20 TEA-Cl, 10 HEPES, 5 $\mathrm{Na}_{2}$-phosphocreatine, 5 ATP-Mg, 0.3 GTP, pH 7.3, with $\mathrm{CsOH}$. The pipette solution was supplemented with varying concentrations of the $\mathrm{Ca}^{2+}$ buffer EGTA $(0.5 \mathrm{mM}$ or 5 $\mathrm{mM}$ ). During experiments, slices were continuously perfused with $1 \mu \mathrm{M}$ TTX, $1 \mathrm{mM}$ 4-AP, and $40 \mathrm{mM}$ TEA-Cl to suppress voltage-gated $\mathrm{Na}^{+}$and $\mathrm{K}^{+}$currents. Junction potentials were not corrected.

Changes in membrane capacitance were monitored using the Sine+DC technique (Neher, 1998) with a software lock-in amplifier (implemented in HEKA Pulse/PatchMaster) by adding a $1 \mathrm{kHz}$ sine-wave voltage command (amplitude \pm 35 $\mathrm{mV}$ ) to $V_{\mathrm{h}}=-80 \mathrm{mV}$. Off-line analysis was done with Igor Pro (WaveMetrics, USA). To avoid a contamination of $\Delta C_{\mathrm{m}}$ estimates after long-lasting presynaptic depolarizations with small $C_{\mathrm{m}}$ transients unrelated to vesicle exocytosis (Yamashita et al., 2005), $\Delta C_{\mathrm{m}}$ was estimated from the averaged $C_{\mathrm{m}}$ values during $50 \mathrm{~ms}$ to $100 \mathrm{~ms}$ after the end of the depolarizations. The number of vesicles were estimated by the following equation: $\mathrm{N}=\Delta \mathrm{Cm} /\left(\mathrm{Cm}_{\mathrm{s}} \cdot \pi \mathrm{d}^{2}\right)$, where $\mathrm{Cm}_{\mathrm{s}} \cdot \pi \mathrm{d}^{2}$ is the average capacitance contribution from a single vesicle by given the values of typical membrane capacitance $\left(\mathrm{Cm}_{\mathrm{s}}\right)$ with $10 \mathrm{fF} / \mu^{2}$ and an averaged synaptic vesicle diameter (d) with $50 \mathrm{~nm}$, yielding an estimation of single vesicle capacitance contribution of $80 \mathrm{aF}$ (Sun and $\mathrm{Wu}, 2001)$. Presynaptic recordings with a leak current $>200 \mathrm{pA}$ were excluded from the analysis.

Presynaptic calyceal action potentials (APs) were elicited by afferent fiber stimulation via a bipolar stimulation electrode placed halfway between the brainstem midline and the MNTB. Stimulation pulses (100 $\mu$ s duration) were applied using a stimulus isolator unit (A.M.P.I., Jerusalem, Israel), with the output voltage set to 1-2 V above threshold for AP generation $(\leq 25 \mathrm{~V})$. APs were measured in the current-clamp mode of the EPC-10 after adjusting the fast-capacitance cancellation while in cell-attached mode. 
For measuring calyceal APs, pipettes were filled with a solution consisting of the following (in $\mathrm{mM}$ ): $100 \mathrm{~K}$-gluconate, $60 \mathrm{KCl}, 10 \mathrm{HEPES}, 0.5$ EGTA, 5 $\mathrm{Na}_{2}$-phosphocreatine, 4 ATP-Mg, $0.3 \mathrm{Na}_{2}$-GTP, pH 7.3 with $\mathrm{KOH}$.

\section{Postsynaptic recordings:}

For postsynaptic recordings, patch pipettes were pulled from thin-walled glass (World Precision Instruments, USA) on a PIP-5 puller (HEKA Elektronik, Germany). Open tip resistance was 2.5-3.5 M $\Omega$. Access resistance $\left(R_{\mathrm{S}}\right)$ ranged from 4 to $7 \mathrm{M} \Omega$ which is essential for voltage-clamp recordings of the large EPSC amplitudes. $R_{\mathrm{S}}$ compensation was set to $\geq 84 \%$ ( $2 \mu$ s delay). The holding potential was $-70 \mathrm{mV}$. For measuring postsynaptic APs, pipettes were filled with a solution consisting of the following (in $\mathrm{mM}$ ): $100 \mathrm{~K}$-gluconate, $60 \mathrm{KCl}, 5 \mathrm{Na}_{2}$-phosphocreatine, $10 \mathrm{HEPES}, 5$ EGTA, $0.3 \mathrm{Na}_{2}-\mathrm{GTP}$ and 4 ATP-Mg, $\mathrm{pH} 7.3$, with $\mathrm{KOH}$. For EPSC recordings, pipettes were filled with a solution consisting of the following (in $\mathrm{mM}$ ): 140 Cs-gluconate, 20 TEA-Cl, 10 HEPES, 5 EGTA, $5 \mathrm{Na}_{2}$-phosphocreatine, 5 ATP-Mg, 0.3 GTP, $\mathrm{pH} 7.3$, with $\mathrm{CsOH}$. During experiments, slices were continuously perfused with normal aCSF solution. No corrections were made for liquid junction potentials.

\subsection{Immunostaining (experiments performed by Dr. Meike Pedersen)}

For whole mounts, freshly dissected brains from P8, P16 and P21 $\mathrm{CPXI}^{+/+}$and $\mathrm{CPXI}^{-/-}$ littermates were gently removed and fixed at room temperature for $2 \mathrm{~h}$ in $4 \%$ paraformaldehyde. Thereafter, the brains were immersed overnight at $4{ }^{\circ} \mathrm{C}$ in $4 \%$ paraformaldehyde PBS. Fifty-micrometer-thick coronal sections of the MNTB were cut at $0{ }^{\circ} \mathrm{C}$ and placed onto SuperFrost microscope slides.

After cutting, the MNTB slices were washed three times for 10 min each time in PBS and incubated for $1 \mathrm{~h}$ in goat serum dilution buffer (GSDB) (16\% normal goat serum, $450 \mathrm{mM} \mathrm{NaCl}, 0.3 \%$ Triton $\mathrm{X}-100,20 \mathrm{mM}$ phosphate buffer, $\mathrm{pH} 7.4$ ) in a wet chamber at room temperature. Primary antibodies were dissolved in GSDB buffer and applied overnight at $4{ }^{\circ} \mathrm{C}$ in a wet chamber. After washing three times for 10 min each time (wash buffer: $450 \mathrm{mM} \mathrm{NaCl}, 20 \mathrm{mM}$ phosphate buffer, 0.3\% Triton X-100), the tissue was incubated with secondary antibodies in GSDB in a wet, light-protected chamber for $1 \mathrm{~h}$ at room temperature. Then the preparations were washed three times 
for $10 \mathrm{~min}$ each time in wash buffer and one time for $10 \mathrm{~min}$ in $5 \mathrm{mM}$ phosphate buffer, placed onto the glass microscope slides with a drop of fluorescence mounting medium (Dako), and covered with thin glass coverslips. The following antibodies were used: mouse IgG1 anti-CtBP2 (BD Biosciences; 1:200), rabbit anti-glutamate receptors 2 and 3 (GluR2/3) (Millipore Bioscience Research Reagents; 1:200), rabbit anti-CPXI/II (Synaptic Systems; 1:300-700), guinea pig anti-vesicular glutamate transporter 1 (VGLUT1) (Synaptic Systems; 1:4000), and secondary Alexa Fluor 488and Alexa Fluor 568-labeled antibodies (Invitrogen; 1:200).

2.5 Confocal microscopy and image analysis (experiments performed by Dr. Meike Pedersen)

Confocal images were acquired using a laser-scanning confocal microscope (Leica TCS SP5, Leica Microsystems CMS) with $488 \mathrm{~nm}$ (Ar) and $561 \mathrm{~nm}(\mathrm{He}-\mathrm{Ne}$ ) lasers for excitation and $10 \times$ air or $40 \times / 63 \times$ oil-immersion objectives. To produce three-dimensional reconstructions of the specimen, a $z$-axis stack of two-dimensional images was taken with a step size of $0.2 \mu \mathrm{m}$ at a pixel size of $0.09 \times 0.09 \mu \mathrm{m}^{2}$. Images were processed using ImageJ and assembled for display in Adobe Photoshop and Illustrator software.

2.6 Immunoblotting (experiments performed by Dr. Kerstin Reim)

Brain tissue extracts of the MNTB from P8, P16, P21 C57BL/6N wt mice and P21 CPXI heterozygote and ko mice (5-8 animals per age group) were analyzed by SDS-PAGE and western blotting using polyclonal rabbit antibodies to Cplx1/2 (1:2500) (Reim et al., 2005). By using fluorescently labeled secondary antibodies, signal intensities were estimated on an Odyssey Infrared Imaging System (LI-COR Biosciences, Bad Homburg, Germany). Expression levels were normalized to Actin (1:4000, Sigma, Hamburg, Germany), which served as loading controls. All western blots experiments were repeated three times. 


\subsection{Reverse transcription and real-time PCR}

Brain tissue of the cochlear nucleus region was dissected from P16-18 C57BL6 mice (5-8 animals per experiment), followed by total RNA extraction with TRIzol reagent (Invitrogen). Reverse transcription $\left(1 \mathrm{~h}\right.$ at $42{ }^{\circ} \mathrm{C}$ and $10 \mathrm{~min}$ at $\left.70{ }^{\circ} \mathrm{C}\right)$ of the total RNA (800 ng-1 $\mu$ g per sample) was performed in first-strand cDNA synthesis mix containing the following (after the final dilution) (in $\mathrm{mM}$ ): 50 Tris- $\mathrm{HCl}, 75 \mathrm{KCl}, 5$ $\mathrm{MgCl}_{2}$, and 5 DTT adjusted to $\mathrm{pH} 8.3$ and $100 \mathrm{U}$ of SuperScript II reverse transcriptase (Invitrogen), $40 \mathrm{U}$ of RNaseOUT ribonuclease inhibitor (Invitrogen), as well as $12.5 \mathrm{ng} / \mu \mathrm{l}$ oligo-dT primers (Invitrogen). Real-time PCR reaction was performed in triplicate for each gene transcript using the Qiagen QuantiTect master mix. Quantitative PCR (qPCR) was done using a Mastercycler ep realplex (Eppendorf) system, with CPXI-IV probes (QT02332687;QT00494662; QT00123025; QT00133805) and the house keeping gene hprt1 (F: Sequence (5'-3'): CTG GTG AAA AGG ACC TCT CGA AG; R: Sequence (5'-3'): CCA GTT TCA CTA ATG ACA CAA ACG) with temperature cycles of $95^{\circ} \mathrm{C}$ for $10 \mathrm{~min}$ initially, followed by 40 cycles at $95{ }^{\circ} \mathrm{C}(15 \mathrm{~s}$ each $)$ and $60{ }^{\circ} \mathrm{C}(1 \mathrm{~min}$ each $)$. In the fluorescence reporter plot, the cycle threshold (CT) was defined in the exponential phase of the amplification curve, at a level of 0.2 fluorescence units. A plot of CT value versus the logarithm of the input amount of total RNA was fitted with a line to estimate the amplification efficiency of each probe. All quantitative PCR experiments were repeated by three times. 


\section{Results}

\subsection{Quantitative analysis of CPX expression in the MNTB region.}

\subsubsection{CPXI is the predominant complexin isoform at calyx of Held}

Complexins are encoded by Cplxl-4 genes which drive the expression of four isoforms (CPXI to IV) in the mammalian CNS (McMahon et al., 1995; Reim et al., 2001). CPXI has $86 \%$ protein sequence identity with CPXII. These two isoforms are ubiquitously expressed in most brain regions and the expression patterns generally overlap. On the other hand, CPXIII and IV are predominantly expressed in ribbon synapses. During development, CPXs are first detectable at P6 in the rat brain and their expression increases to reach a plateau at around 20 days after birth when most synapses have been formed (Ishizuka et al., 1997; Reim et al., 2005). To clarify whether CPXI is the predominant isoform at the calyx of Held synapse, we performed western blot analysis to quantify the protein expression. Protein extracts were obtained from the $\mathrm{MNTB}$ regions of $\mathrm{P} 17 \mathrm{CPXI}^{-/-}$and $w t$ mice. For comparison, protein extracts from whole brain and retina were additionally included in the analysis. These experiments revealed that among all complexins CPXI is predominantly expressed in the MNTB region whereas only a minor expression of CPXII could be detected (Fig. 1). The antibody against CPXI cross-reacts with CPXII, but both proteins can be well discriminated by molecular weight. CPXIII or IV expression were not detectable in the MNTB region, but was abundant in the retina. Notably, the expression of CPXI was completely abolished in $\mathrm{CPXI}^{-/-}$mice and no compensatory 
increase of CPXII was observed. We also examined the expression levels of several key synaptic proteins including Syntaxin 1, SNAP25, Synaptobrevin 2 to access possible changes in protein composition in CPXI-deficient synapses. As seen in Fig. 1, the expression levels of these proteins were unaltered.

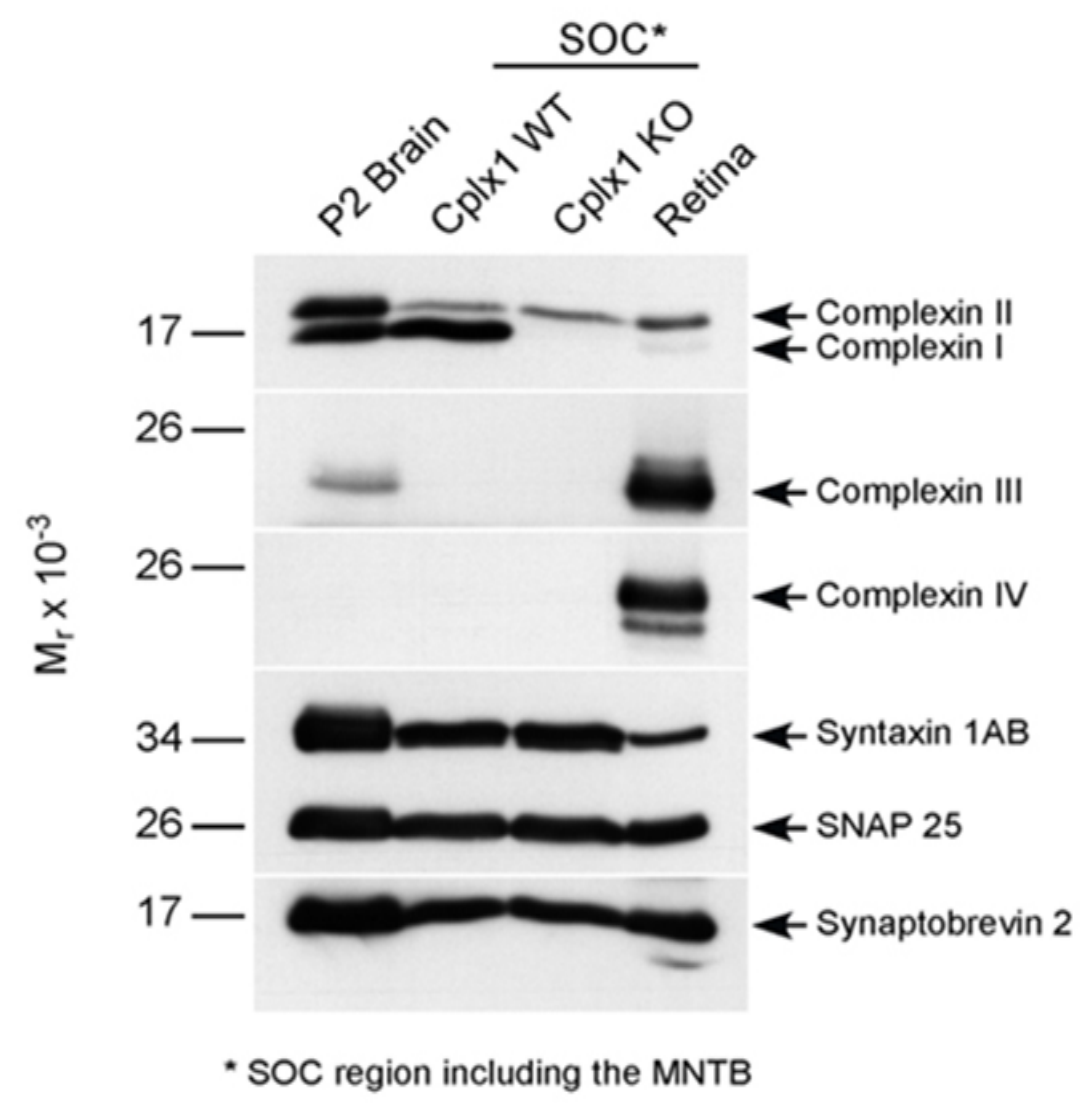

Figure1: Quantitative analysis of CPXs expression in the MNTB region.

Protein expression was analyzed by SDS-PAGE and using an anti-CPXI/II antibody and selected antibodies for presynaptic proteins as internal control including Synataxin1ab, Synaptobrevin2, and SNAP-25. Homogenates of MNTB region from $\mathrm{P} 17 \mathrm{CPXI}^{+/+}$and $^{-/-}$mice and retina $(10 \mu \mathrm{g}$ per lane) were prepared for 3 replicates for each of the indicated $\mathrm{CPXI}^{+/+}$and ${ }^{-/-}$genotypes. Positions of CPXI and CPXII protein are indicated. ( $n=3$; technical replicates)

Because the protein extract of the MNTB region is composed of pre- as well as postsynaptically expressed proteins, the described expression pattern may not necessarily reflect the protein expression levels in the presynaptic calyx terminals. Therefore, we additionally employed quantitative real-time PCR to examine the CPX 
expression pattern at the transcriptional level. Because calyx terminals originate from the globular bushy cells in the contralateral ventral cochlear nuclease (AVCN) (Harrison and Irving, 1966; Kuwabara et al., 1991), we dissected the ACVN region from P16-18 wt mice in order to collect mRNA from bushy cell somata. After mRNA extraction followed by reverse transcription, qPCR analysis was performed. CpxI, cpxII, cpxIII and cpxIV probes were included in the reactions in order to validate the relative expression levels (Fig. 2). Fig. 2 illustrates that the expression level of cpx I was 12 times higher than that of $c p x I I(\mathrm{p}<0.01$, t-test), while $c p x I I I / I V$ expression was virtually undetectable. Taken together, these results suggest that CPXI is the dominant complexin isoform expressed at the calyx of Held terminal.

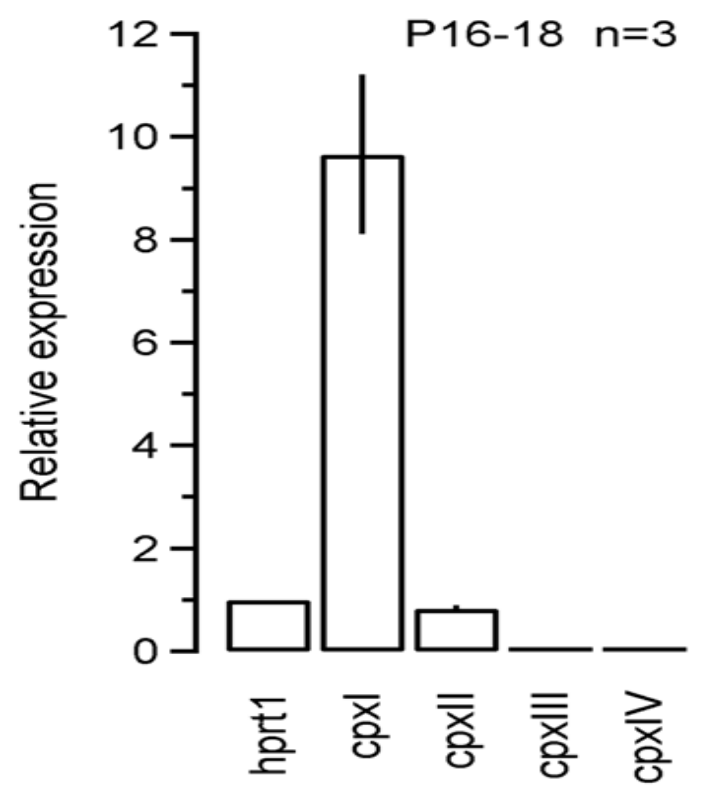

Figure2: Validation of mRNA expression level of different CPX isoforms. Quantitative real-time PCR data were analyzed. The expression level was calculated according to $2^{-\left(\mathrm{C}_{\mathrm{t}} \text { gene-C }\right.} \mathrm{t}_{\mathrm{t}}$ rtl $)$, where $\mathrm{C}_{\mathrm{t}}$ gene and $\mathrm{C}_{\mathrm{t}}$ hprtl represent the thresholds of detection for the genes tested and for the housekeeping gene, respectively. The relative expression was then obtained by normalizing the expression levels to that of the housekeeping gene hprt1 ( $n=3$; technical replicates). 


\subsubsection{CPXI is pre- and postsynaptically expressed in the MNTB region}

To characterize the cellular and subcellular distribution of CPXs in the MNTB, we performed immunocytochemistry experiments, using specific antibodies against $\mathrm{CpxI} / \mathrm{II}$ and the presynaptic marker protein vGluT1 (vesicular glutamate transporter 1). Immunofluorescence images are shown in Fig. 3 where the green fluorescence marks the expression of $\mathrm{CpxI} / \mathrm{II}$, and red marks vGluT1 expression. Because both presynaptic calyces as well as postsynaptic principal neurons were labeled by the anti-CpxI/II antibody, we can conclude that $\mathrm{CpxI} / \mathrm{II}$ is expressed pre- and postsynaptically within the MNTB. Nevertheless, CpxI/II is strongly expressed in P14 and P21 calyx terminals as indicated by the colabeling with the anti-vGluT1 antibody. The abundant expression CPXI in the MNTB principle cells raises the possibility that genetic ablation of CPXI may not only affect pre- but also postsynaptic function .

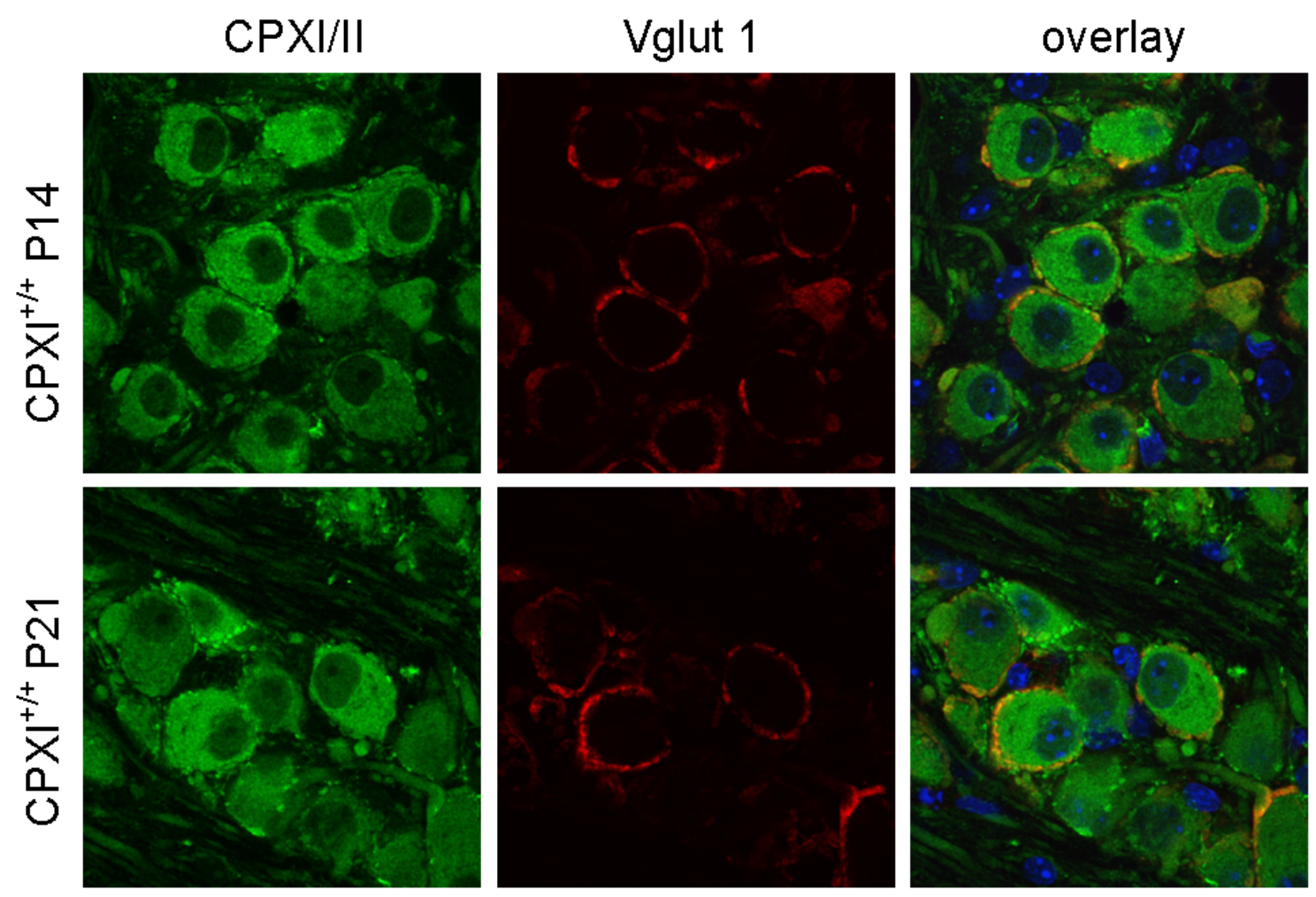


Figure3: CPXI/II localization patterns in the developing calyx of Held. Immunofluorescence images representing projections of confocal sections of MNTB of $\mathrm{CPXI}^{+/+}$mice co-stained with an anti-CPXI/II antibody (green, left panels), and an anti-Vglut 1 antibody (red, middle panels) at postnatal ages P14 and P21. The right panels represents the corresponding overlay images.

\subsection{Evaluation of synaptic strength in CPXI-deficient calyx synapses}

After genetic ablation of CPXI expression in the calyx of Held without apparent compensatory up-regulation of other complexins (Fig. 1), we are able to study its function in synaptic transmission at this synapse. To this end, we recorded AP-evoked EPSCs elicited by afferent fiber stimulation in wt and $\mathrm{CPXI}^{-/-}$synapses from $\mathrm{P} 8$ to P30 mice to test for changes in synaptic strength. Representative examples of EPSCs recorded at three different ages in $w t$ and $\mathrm{CPXI}^{-/-}$mice at different holding potentials are illustrated in Fig. 4A. Synaptic transmission was intact at all ages tested and synaptic strength was unchanged in $\mathrm{P} 8 \mathrm{CPXI}^{-/-}$synapses when compared to control (Fig. 4A1). However, EPSC amplitudes of $\mathrm{CPXI}^{-/-}$synapses started to decline at the age of P14 (Fig. 4B). At the ages of P20 and P30, EPSC amplitudes of $\mathrm{CPXI}^{-/-}$ synapses were strongly reduced compared to those of wt mice (Fig. 4A2, A3).

Fig. 4B illustrates AP-evoked EPSC amplitudes plotted as a function of postnatal age for a wide range of developmental stages ranging from P14 to P21. Linear regression analysis of the scatter plot revealed a gradual decline of the average peak EPSC amplitude in $\mathrm{CPXI}^{-/-}$synapses from $-7.24 \pm 0.72 \mathrm{nA}(\mathrm{P} 14)$ to $-3.48 \pm 0.23 \mathrm{nA}(\mathrm{P} 21)$. In contrast, the average peak EPSC amplitudes of wt mice steadily increased with maturation from $-10.9 \pm 2.71 \mathrm{nA}$ to $-14 \pm 0.34 \mathrm{nA}$, similarly as previously reported 
(Taschenberger and von Gersdorff, 2000). Although there was a large cell to cell variability, the EPSC amplitudes were $\sim 37 \%$ reduced in $\mathrm{P} 16-\mathrm{P} 21 \mathrm{CPXI}^{-/-}$mice with an average of $-4.37 . \pm 0.04 \mathrm{nA}$ compared to wt mice $-11.94 \pm 0.06 \mathrm{nA}(\mathrm{p}<0.001$, $\mathrm{t}$-test $)$.
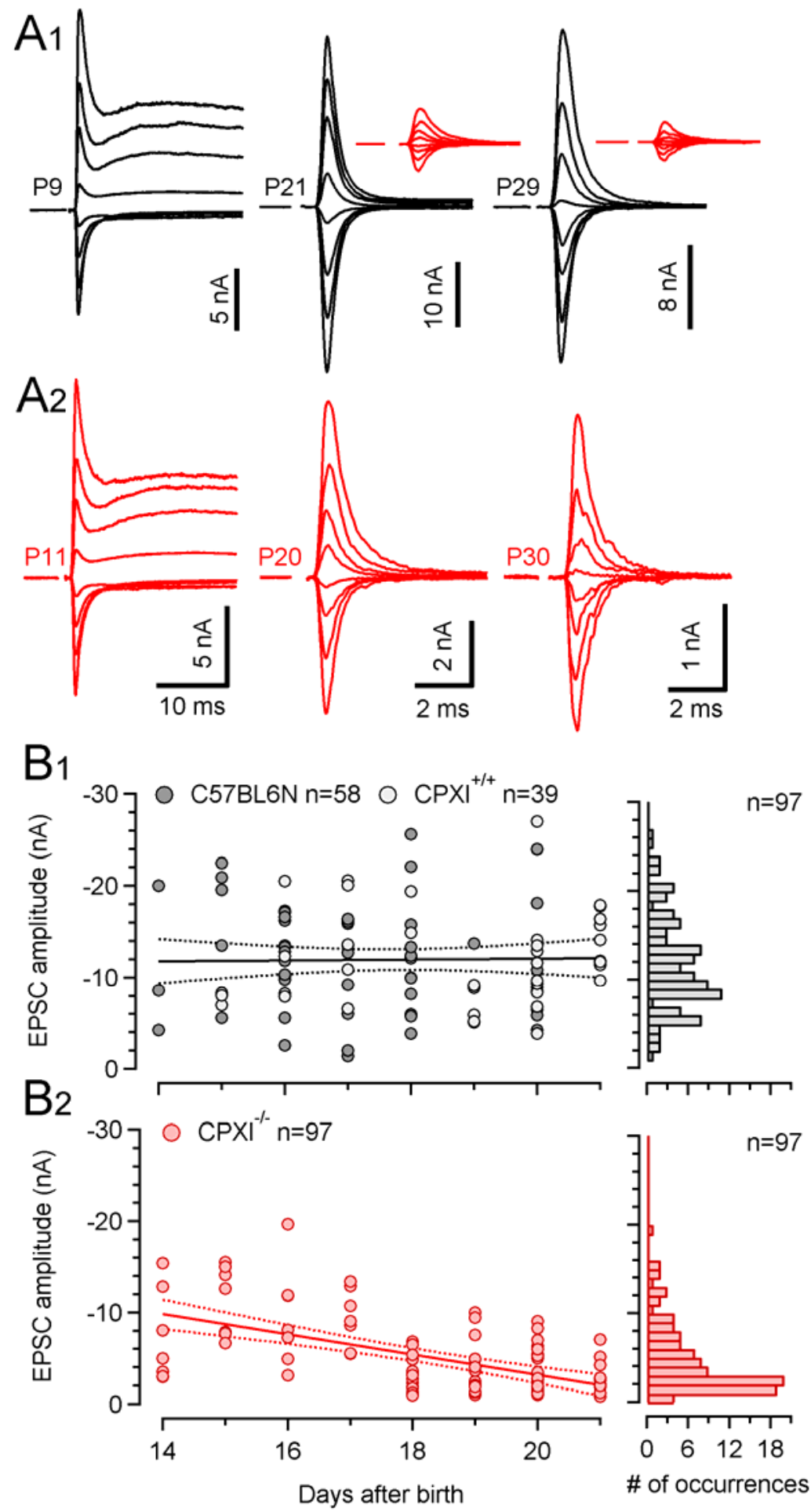
Figure 4. Developmental declined synaptic strength in synapses of $\mathrm{CPXI}^{-/-}$mice

A, Families of representative AMPA receptor-mediated EPSCs recorded at $\mathrm{V}_{\mathrm{h}}=-70$ $\mathrm{mV}$ to $+70 \mathrm{mV}(20 \mathrm{mV}$ increments) and evoked by afferent fiber stimulation in synapses of $\mathrm{CPXI}^{+/+}$mice at P9, P21 and P29 (A1) and $\mathrm{CPXI}^{-/-}$mice at P11, P20, and P29 (A2). Insets in the top right and top middle panels show the same traces from $\mathrm{CPXI}^{-/-}$drawn at scale for comparison. B, Left: Scatter plots of AMPA EPSC peak amplitudes versus age for wildtype (B1) and $\mathrm{CPXI}^{-/-}$(B2) mice. Right: Corresponding frequency distribution of EPSC peak amplitudes. Solid and dotted lines represent linear regressions and 95\% confident intervals, respectively.

\subsection{The time course of calyceal EPSCs}

When comparing EPSC waveforms of $\mathrm{CPXI}^{-/-}$synapses with those of wt synapses, we noticed that the time course of the EPSC in CPXI deficient mice was slightly slower than that in wt mice (Fig. 5A). This observation prompted us to analyze the EPSC kinetics of CPXI-deficient synapses in more detail. During early postnatal development of calyx synapses, the decay time course of AMPA receptor (AMPAR)-mediated EPSCs accelerates markedly. This acceleration of the EPSC kinetics is mainly due to a composition switching of AMPA receptors during development and has been shown to play an indispensable role in the refinement of high-fidelity transmission at mature calyx of Held synapse (Iwasaki and Takahashi, 2001; Koike-Tani et al., 2005; Taschenberger and von Gersdorff, 2000). We found the kinetics of EPSCs in $\mathrm{CPXI}^{-/-}$mice slightly but consistently slower compared to that of wt synapses as indicated by the slower rise times $\left(154 \pm 3 \mu \mathrm{s}\right.$ in $\mathrm{CPXI}^{-/-}$mice versus $147 \pm 2 \mu \mathrm{s}$ in wt mice) and slower weighted decay time constants $\left(553 \pm 4 \mu \mathrm{s}\right.$ in $\mathrm{CPXI}^{-/-}$ synapses versus $440 \pm 2 \mu \mathrm{s}$ in wt mice, Fig. 5D,E). Because the time course of AP-evoked EPSCs is determined in part by the time course of vesicle release (Diamond and Jahr, 1995) in addition to the postsynaptic AMPAR kinetics, it is possible that the genetic ablation of CPXI affects the synchrony of the vesicle release 
process in $\mathrm{CPXI}^{-/-}$synapses. Changes in EPSC kinetics during development are summarized in Fig. 5B,C. Since synaptic transmission was not altered in $\mathrm{CPXI}^{-/-}$mice before P12, we primarily focused on the characterization of synaptic transmission in post-hearing $\mathrm{CPXI}^{-/-}$mice $(\mathrm{P} 16-21)$ in this study.

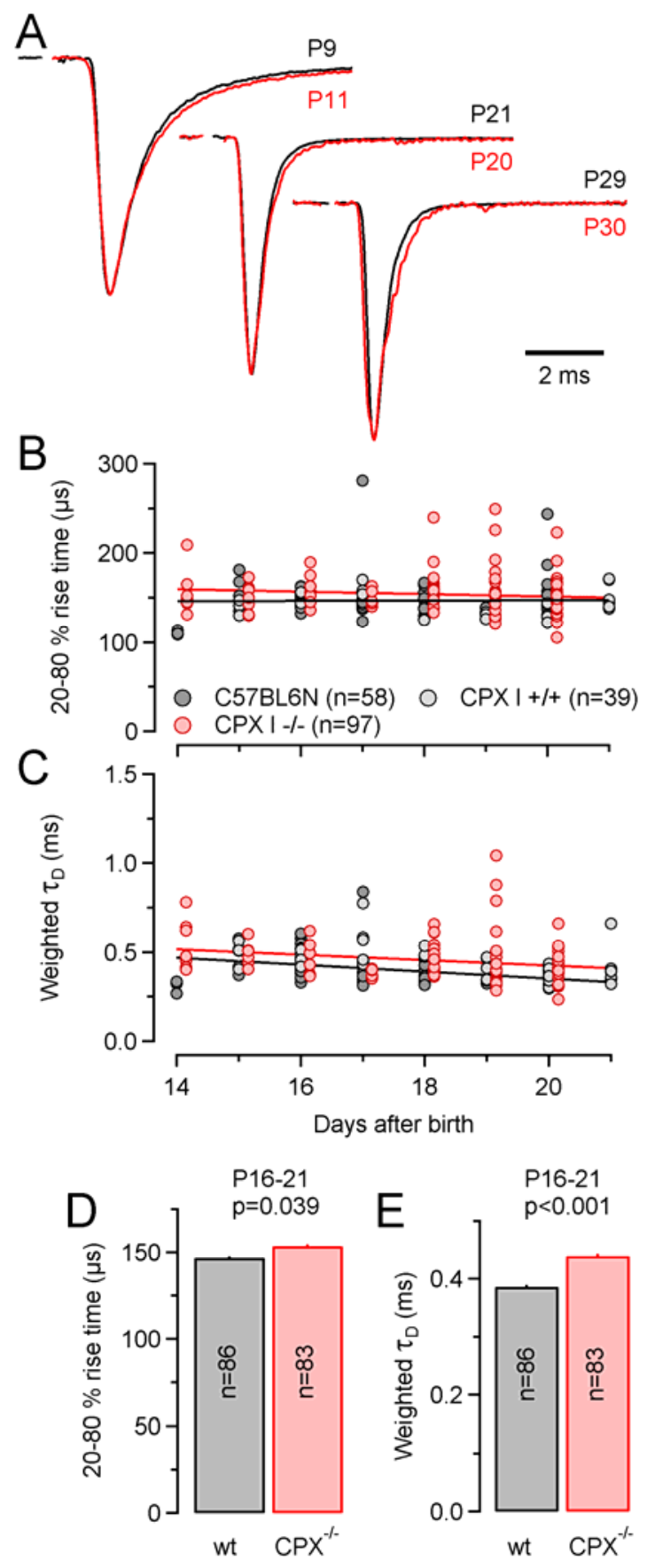


Figure 5: Slightly slower time course of AP-evoked EPSCs in $\mathrm{CPXI}^{-/-}$and wildtype mice throughout postnatal development.

A, Representative traces of evoked EPSCs from wt (black) and $\mathrm{CPXI}^{-/}$(red) mice of three different age groups (P9-11, P20-21 and P29-30) were normalized and superimposed. Same cells as shown in Fig. 1. B,C, Scatter plots summarizing rise times (B) and weighted decay time constants (C) of AP-evoked EPSCs analyzed from P14-21 CPXI ${ }^{+/+}$( $\mathrm{n}=39$, light grey circles) and C57BL6N ( $=58$, dark grey circles) and $\mathrm{CPXI}^{-/-}$( $\mathrm{n}=97$, red circles) mice. $\mathrm{CPXI}^{-/-}$mice show slightly slower kinetics compared to $w t$ mice throughout postnatal development. D,E, Mean rise times and weighted decay time constants. Bar graphs represent summary data obtained from P16-21 wt (gray) and $\mathrm{CPXI}^{-/}$(red) mice. Number of cells as indicated. Both rise times as well as weighted decay time constants were only slightly, but statistically significantly, slower in $\mathrm{CPXI}^{-/-}$(red) mice $(\mathrm{p}<0.001)$.

\subsection{Mechanisms that underlie the reduced synaptic strength in $\mathrm{CPXI}^{-/}$calyces}

Many mechanisms upstream or downstream of vesicle fusion have been shown to participate in regulating synaptic strength (von Gersdorff and Borst, 2002), including changes in presynaptic $\mathrm{Ca}^{2+}$ influx and/or AP waveform which both can result in altered presynaptic release probability (p), alterations in the size of readily releasable pool of synaptic vesicles (n) or changes in postsynaptic quantal size (q). Previous studies suggest that CPXI deletion primarily affect presynaptic release probability (Reim et al., 2001; Strenzke et al., 2009). In none of these studies, presynaptic properties such as $\mathrm{Ca}^{2+}$ influx, AP waveform or RRP size could be directly measured. To clarify the mechanisms underlying the reduced synaptic strength in $\mathrm{CPXI}^{-/-}$calyx synapses, we studied presynaptic $\mathrm{Ca}^{2+}$ currents by voltage-clamp recordings from presynaptic terminals.

\subsubsection{Presynaptic $\mathrm{Ca}^{2+}$ influx}

In calyx of Held terminals, the AP-triggered $\mathrm{Ca}^{2+}$ influx is mediated by $\mathrm{N}-, \mathrm{P} / \mathrm{Q}-$ and R-types $\mathrm{Ca}^{2+}$ channels. During postnatal synapse maturation, P/Q type channels become the predominant channel subtype at the calyx of Held (Iwasaki et al., 2000). 
In order to test if genetic deletion of CPXI expression would alter $\mathrm{Ca}^{2+}$ channel function, we recorded presynaptic voltage-gated $\mathrm{Ca}^{2+}$ currents $\left(\mathrm{I}_{\mathrm{Ca}(\mathrm{V})}\right)$ by voltage-clamping presynaptic terminals of $w t$ and $\mathrm{CPXI}^{-/-}$mice. As shown in Fig. 6D, the peak amplitude of $\mathrm{I}_{\mathrm{Ca}(\mathrm{V})}$ in $\mathrm{CPXI}^{-/}$mice was unchanged compared to wt mice with an average of $-1.06 \pm 0.07 \mathrm{nA} ; \mathrm{n}=30(\mathrm{P} 8-12)$ and $-1.53 \pm 0.12 \mathrm{nA} ; \mathrm{n}=18(\mathrm{P} 16-20)$ $\left(\mathrm{CPXI}^{+/+}: \mathrm{P} 8-\mathrm{P} 12, \mathrm{n}=27:-0.96 \pm 0.05 \mathrm{nA}\right.$ and P16-P20, n=13: $\left.-1.41 \pm 0.15 \mathrm{nA}\right)$. This result suggests that CPXI is not involved in regulating number or properties of presynaptic $\mathrm{Ca}^{2+}$ channels.

\subsubsection{The size of the readily releasable pool (RRP)}

Next, we investigate whether the size of the pool of readily releasable vesicles is altered in $\mathrm{CPXI}^{-/-}$synapses. Different methods have been used to estimate the RRP size at different synapses which mostly rely on measuring vesicle release following strong presynaptic $\mathrm{Ca}^{2+}$ influx resulting in a depletion of the RRP. Here, we used presynaptic capacitance measurements $\left(\Delta C_{\mathrm{m}}\right)$ by directly voltage-clamping presynaptic terminals of $\mathrm{wt}$ and $\mathrm{CPXI}^{-/-}$mice and evoking release by step depolarizations of 1 to $50 \mathrm{~ms}$ duration (from $\mathrm{V}_{\mathrm{h}}=-80$ to $0 \mathrm{mV}$ ). Example traces obtained by such experiments are shown in Fig. $6 \mathrm{~A} . \Delta C_{\mathrm{m}}$ values were converted into vesicle numbers by assuming a single vesicle capacitance of $80 \mathrm{aF}$. Considering that the reduction of synaptic strength in $\mathrm{CPXI}^{-/-}$mice appeared relatively late during development, we categorized the experiments into two age groups: P8-12 and P16-20. In Fig. $6 \mathrm{C}, \Delta \mathrm{C}_{\mathrm{m}}$ values were plotted against the duration of the presynaptic depolarization. Initially, $\Delta \mathrm{C}_{\mathrm{m}}$ increased steeply with longer depolarizations until about $10 \mathrm{~ms}$. Longer steps resulted in a further but less steep increase in $\Delta \mathrm{C}_{\mathrm{m}}$ such that the curve was well fit by a bi-exponential function. Such bi-exponential fits resulted in time constants of $\tau_{\text {fast }}=0.99 \mathrm{~ms}$ and $\tau_{\text {slow }}=23 \mathrm{~ms}$ in P16-20 $\mathrm{CPXI}^{-/-}$mice, and $\tau_{\text {fast }}=$ 
$0.89 \mathrm{~ms}$ and $\tau_{\text {slow }}=149 \mathrm{~ms}$ in wt P16-20 mice, suggesting heterogeneity among readily releasable vesicles reminiscent of the fast and slowly releasable pools described by Sakaba and Neher (2001). For simplicity, we considered here the $\Delta \mathrm{C}_{\mathrm{m}}$ measured after a $50 \mathrm{~ms}$ as an estimate of the total RRP (sum of fast and slowly releasable vesicles). This value may be an overestimate if significant vesicle pool replenishment occurs within the $50 \mathrm{~ms}$ step. However, with a replenishment rate constant in the range of seconds, the amount of vesicles newly replenished into the RRP is probably negligible (Lin et al., 2011). On the other hand, ongoing endocytosis will lead to an underestimation of the RRP by $\Delta \mathrm{C}_{\mathrm{m}}$ measurements. After converting the $\Delta \mathrm{C}_{\mathrm{m}}$ into vesicle number, we estimate that $\mathrm{CPXI}^{-/}$terminals harbor a readily releasable pool of $\sim 2725$ vesicles $(218 \pm 12.2 \mathrm{fF}, \mathrm{n}=30)$ at $\mathrm{P} 8-\mathrm{P} 12$ and $\sim 4600$ vesicles $(368 \pm 39.2 \mathrm{fF}, \mathrm{n}=18)$ at $\mathrm{P} 16-\mathrm{P} 21$. These results are similar to those obtained from wt mice with an average $\sim 2562$ vesicle $(207 \pm 17.2 \mathrm{fF}, \mathrm{n}=27)$ and $\sim 4388$ vesicles $(351 \pm$ $51.6 \mathrm{fF}, \mathrm{n}=13$ ), Fig. 6E (Ryugo et al., 1996; Taschenberger et al., 2002). Thus, presynaptic capacitance recordings yielded no evidence in favor of a change in RRP size in $\mathrm{CPXI}^{-/-}$mice, suggesting that the decrease in synaptic strength is mainly caused by a decrease in presynaptic release probability and/or postsynaptic quantal size. 

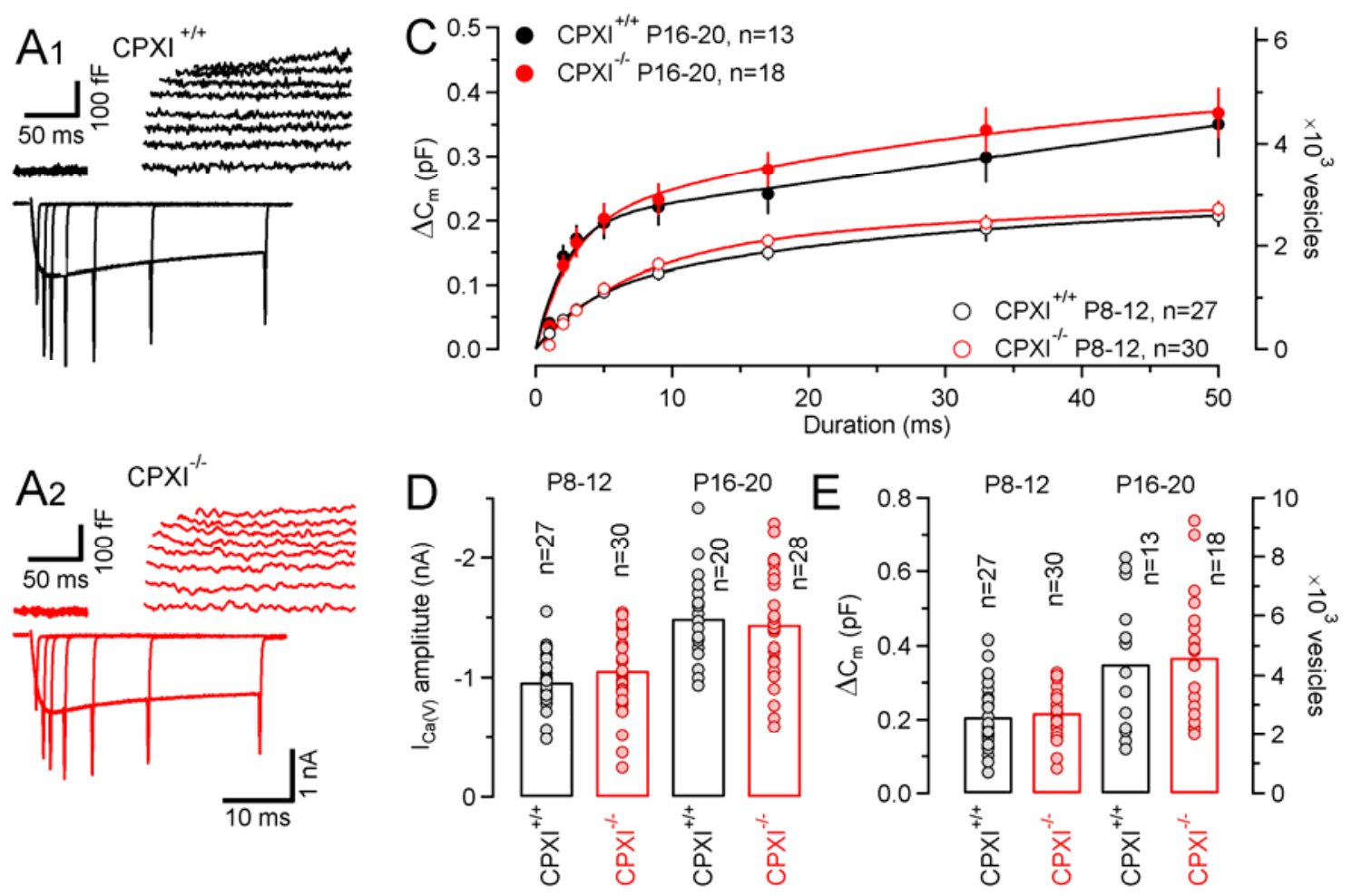

Figure6: Unaltered presynaptic voltage-gated $\mathrm{Ca}^{2+}$ currents and similar readily releasable pool size in $\mathrm{CPXI}^{-/-}$synapses.

A, Vesicle exocytosis triggered by presynaptic $\mathrm{Ca}^{2+}$ influx and recorded by capacitance measurements. Presynaptic $\mathrm{I}_{\mathrm{Ca}(\mathrm{V})}$ and the corresponding changes in membrane capacitance $\left(\Delta \mathrm{C}_{\mathrm{m}}\right)$ evoked by step depolarizations of $1,2,3,5,9,17,33$ and $50 \mathrm{~ms}$ duration from $\mathrm{V}_{\mathrm{h}}=-80$ to $0 \mathrm{mV}$ were recorded with $0.5 \mathrm{mM}$ EGTA in the pipette solution from $\mathrm{CPXI}^{+/+}(\mathbf{A 1})$ and $\mathrm{CPXI}^{-/-}$(A2) terminals. C, Average $\Delta \mathrm{C}_{\mathrm{m}}$ values plotted as a function of the duration of the step depolarizations for $\mathrm{CPXI}^{+/+}$ (black) and $\mathrm{CPXI}^{-/}$(red) terminals. Data was grouped into two developmental stages: P8-10 (open symbols) and P16-20 (filled symbols). $\Delta \mathrm{C}_{\mathrm{m}}$ values were similar for wt and $\mathrm{CPXI}^{-/-}$terminals throughout development. D,E, Averaged peak amplitudes of $\mathrm{I}_{\mathrm{Ca}(\mathrm{V})}(\mathbf{E})$ and $\Delta \mathrm{C}_{\mathrm{m}}$ values (50 ms depolarizations) (F) for $\mathrm{CPXI}^{+/+}$(black) and $\mathrm{CPXI}^{-/-}$ (red) terminals. Number of terminals tested as indicated.

Developmental changes in RRP and $I_{\mathrm{Ca}(\mathrm{V})}$ are plotted in Fig. 7AB. The average RRP size increased from $\sim 2725$ to $\sim 4600$ vesicles from at the age of P8 to P21 (wt, $n=40$ and ko, $n=48$ ), while the average amplitudes of $\mathrm{I}_{\mathrm{Ca}(\mathrm{V})}$ increase by $\sim 50 \%$ over the same period of time. A larger vesicle pool size in mature synapses may allow prolonged 
high-frequency firing at calyx of Held synapse (Taschenberger and von Gersdorff, 2000).
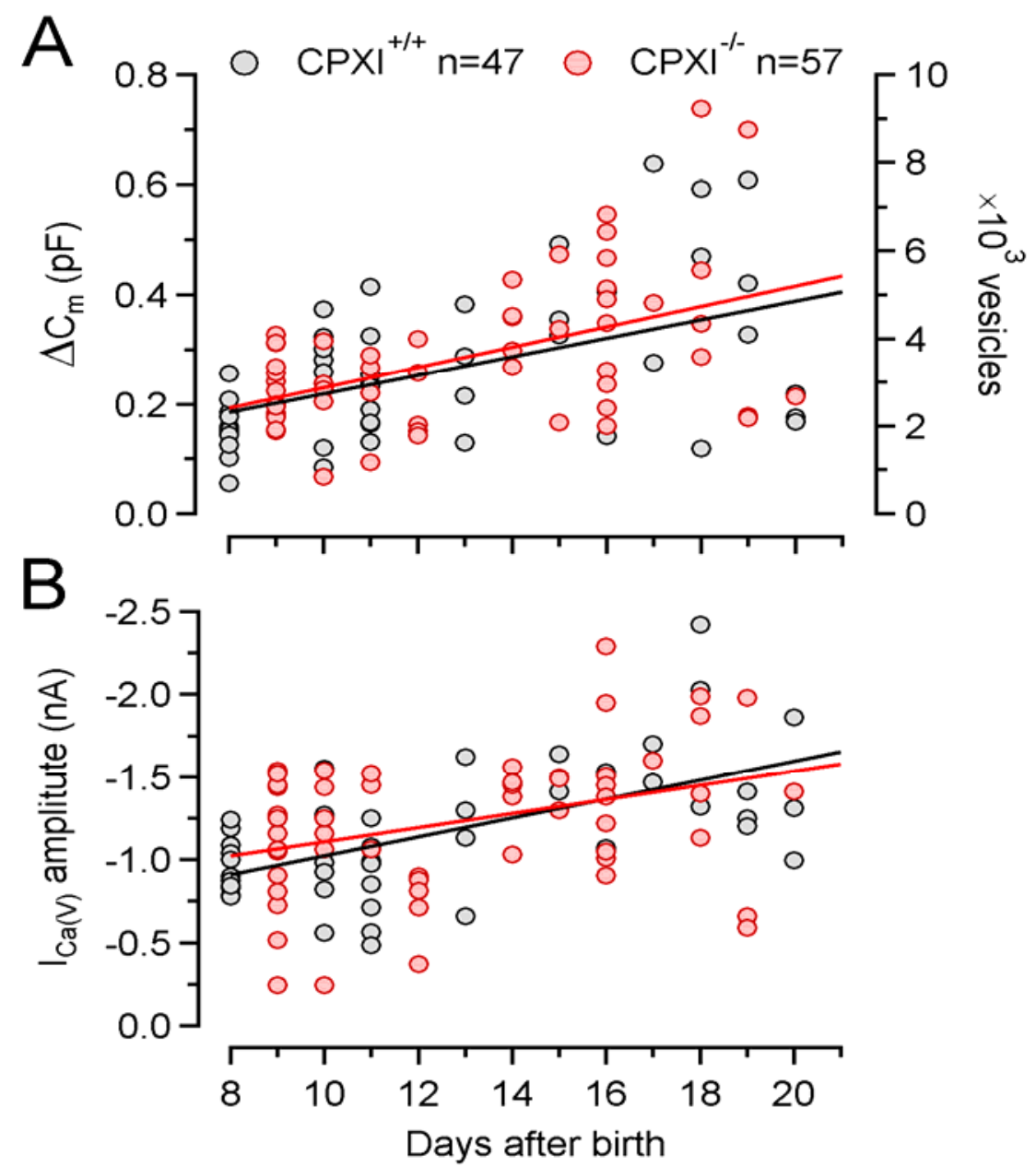

Figure 7: Scatter plot of presynaptic voltage-gated $\mathrm{Ca}^{2+}$ currents and readily releasable pool size over a developmental range of P8 to P21. A,B, Scatter plots of peak amplitudes of $\mathrm{I}_{\mathrm{Ca}(\mathrm{V})}(\mathrm{B})$ and corresponding $\Delta \mathrm{C}_{\mathrm{m}}$ values (obtained from $50 \mathrm{~ms}$ step depolarizations) as a function of postnatal age. Both $\mathrm{I}_{\mathrm{Ca}(\mathrm{V})}$ as well as exocytosis increased similarly in $\mathrm{CPXI}^{+/+}$(gray circles) and $\mathrm{CPXI}^{-/-}$(red circles) terminals with maturation. Number of terminals tested as indicated.

\subsubsection{Comparison of mEPSC amplitudes, kinetics and frequency in $\mathrm{CPX}^{-/-}$and $\mathrm{wt}$ synapses}

According to the quantal theory of synaptic transmission (Katz, 1969), synaptic strength can be regarded as the product of three parameters: release probability $(p)$, 
vesicle number $(N)$ and quantal size $(q)$. Thus, quantal size plays an important role in the regulation of synaptic strength. It can be modulated for example by changing the gluatamate content of synaptic vesicles (Wu et al., 2007) and/or changing number and/or properties of postsynaptic receptors (Bellingham et al., 1998). To test whether the quantal size is affected by the genetic deletion of CPXI expression, we measured spontaneous miniature EPSCs (mEPSCs) in $\mathrm{CPXI}^{-/-}$and wt mice. The amplitude and decay time constant of the mEPSCs were unaltered (amplitudes: $\mathrm{wt}=68.4 \pm 3.1 \mathrm{pA}$ versus $\mathrm{CPXI}^{-/-}=63.6 \pm 2.4 \mathrm{pA}$, decay time constant: wt $=240 \pm 7 \mu$ s versus $\mathrm{CPXI}^{-/-}=$ $238 \pm 6 \mu \mathrm{s}$ ), indicating that removal of CPXI neither affected the vesicle filling nor composition of postsynaptic AMPA receptors (Fig. 8DE). On the other hand, we found that the mEPSC frequency was significantly reduced in $\mathrm{CPXI}^{-/-}$compared to wt synapses with an average frequency $2.1 \pm 0.2 \mathrm{~Hz}, \mathrm{n}=55$ versus $4.4 \pm 0.4 \mathrm{~Hz}, \mathrm{n}=39$ $(p<0.001$, t-test, Fig. 8C). Taken together with the lack of change in RRP size, these findings suggest that the reduction of synaptic strength in $\mathrm{CPXI}^{-/-}$mice was mainly caused by changes in release probability.

When plotting the rate of spontaneous release as a function of age, we found opposite trends for the changes in spontaneous release during development in wt and $\mathrm{CPXI}^{-/-}$ synapses (Fig. 8B). While the mEPSC frequency increased slightly in wt synapses, a decrease with age was found in $\mathrm{CPXI}^{-/-}$synapses. This result of reduced mEPSC frequency in $\mathrm{CPXI}^{-/}$calyx synapses contrasts studies in the Drosophila NMJ where complexin deletion caused a 20 -fold increase in the rate of spontaneous release (Huntwork and Littleton, 2007). Surprisingly, the mEPSC release rate remained elevated for several hundreds of ms after eliciting a single AP-evoked EPSC in $\mathrm{CPXI}^{-/-}$synapses. On average, the mEPSC frequency increased from $1.06 \pm 0.14 \mathrm{~Hz}$ 
before the single EPSC to $2.55 \pm 0.31 \mathrm{~Hz}$ after the single EPSC in $\mathrm{CPXI}^{-/-}$mice $(n=15)$.

In contrast, the average mEPSC frequency decreased slightly from $3.69 \pm 0.50 \mathrm{~Hz}$ to

$3.31 \pm 0.59 \mathrm{~Hz}$ when applying the same experimental protocol in wt synapses $(n=11)$.

These results suggest that newly docked vesicle remain unstable and are more prune to being released prematurely in CPXI-deficient synapses.

$\mathrm{A}_{1}$

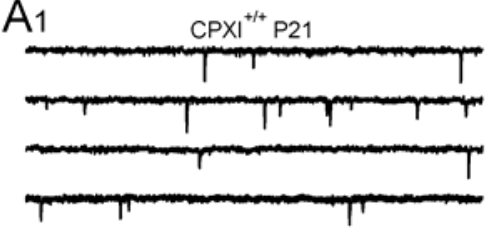

A2 $\quad$ CPXI $^{-1}$ P19
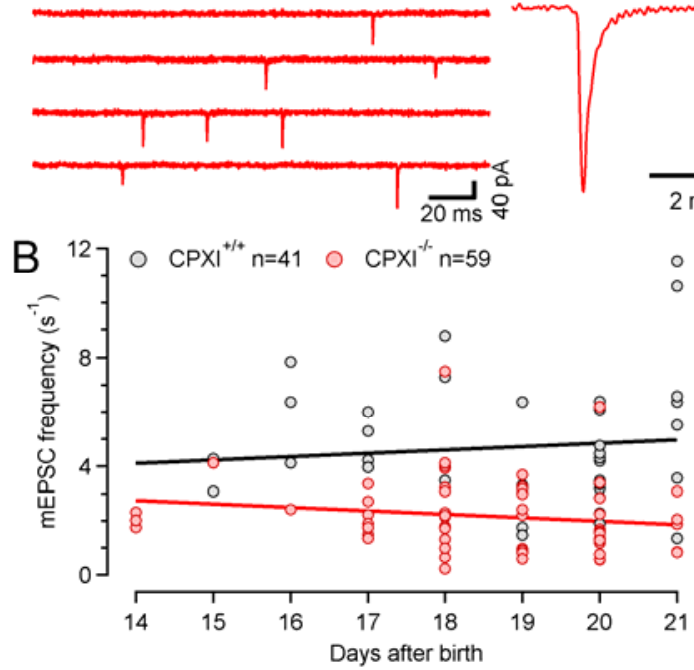
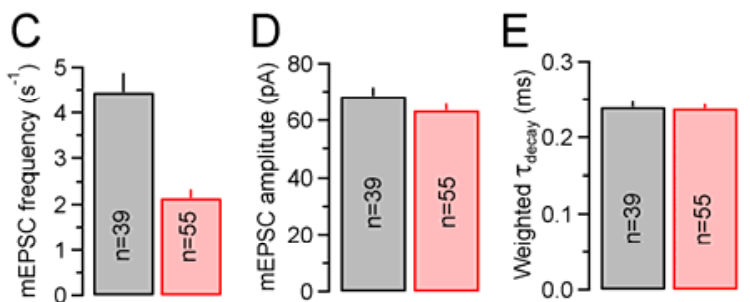

$\mathrm{F}_{2} \mathrm{CPXI}^{-1-}$

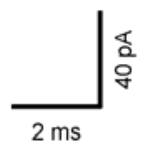

吉 $\mathrm{F}_{1}$
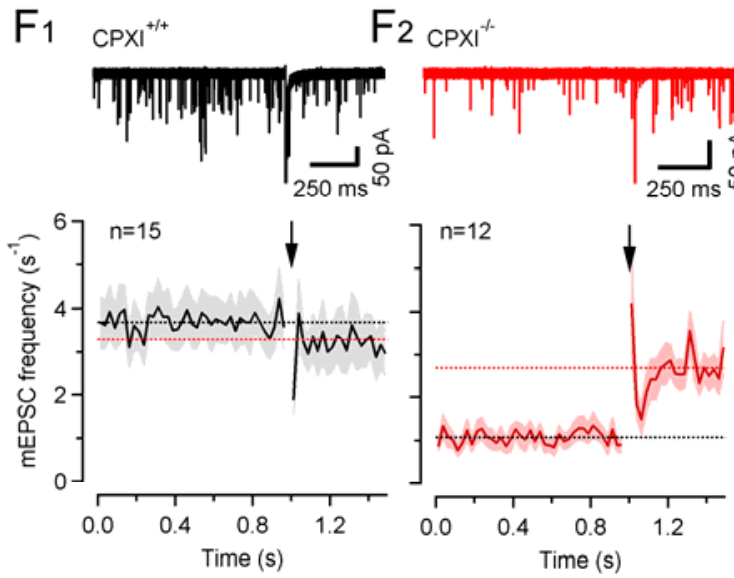

Figure 8: Unaltered mEPSC amplitudes and kinetics but reduced frequency of spontaneous release in $\mathrm{CPXI}^{-/-}$synapses.

A, Baseline recordings showing individual mEPSCs (left panels) and corresponding average mEPSC waveforms (right panels) for a $\mathrm{P} 21 \mathrm{CPXI}^{+/+}$(A1, black) and a P19 $\mathrm{CPXI}^{-/}$mouse (A2, red). B, Scatter plot of spontaneous mEPSCs frequency as a function of age. Solid lines represent linear regressions. Note the contrasting developmental trends in $\mathrm{CPXI}^{+/+}$versus $\mathrm{CPXI}^{-/-}$mice. $\mathbf{C , D}, \mathbf{E}$, Summary data showing average values for mEPSC frequency $(\mathbf{C})$, mEPSC amplitude (D) and mEPSC decay kinetics (E). Number of synapses analyzed as indicated. The average mEPSCs frequency of $\mathrm{CPXI}^{-/-}$mice (red circles) was $\sim 48 \%$ of that in $\mathrm{CPXI}^{+/+}$(grey circles) mice, while mEPSC amplitudes and mEPSC decay time constants were unchanged. (F) Enhanced mEPSC release after eliciting a single AP-evoked EPSC in $\mathrm{CPXI}^{-/}$ synapses. mEPSCs frequencies were monitored $1 \mathrm{~s}$ before and $500 \mathrm{~ms}$ after eliciting a 
single presynaptic AP (top panels, 20 consecutive traces shown superimposed) and average mEPSC frequency values for $25 \mathrm{~ms}$ bins were plotted (bottom panels). Arrows mark the time of the AP-evoked EPSCs which are truncated in the top panels. The mEPSC frequency was slightly reduced after a single AP-induced EPSC in $\mathrm{CPXI}^{+/+}$but strongly enhanced in $\mathrm{CPXI}^{-/-}$mice.

\subsubsection{Comparison of time course of synchronous release transients}

As described above, P16-21 $\mathrm{CPXI}^{-/-}$synapses showed slightly slower EPSC kinetics in comparison to wt synapses. Therefore, we next analyzed the release time course by deconvolution. Deconvolution of AP-evoked EPSCs with average mEPSC waveforms obtained from the same synapses was performed in the frequency domain using fast Fourier transform (FFT) routines yielding the time course of quantal release. As shown in Fig. 9, the peak release rates of wt synapses were about two times higher than those of $\mathrm{CPXI}^{-/-}$synapses with an averaged peak release rate of $823 \pm 62$ vesicles/ms $(n=24)$ and $309 \pm 36$ vesicles/ms $(n=36)$ for wt and $\mathrm{CPXI}^{-/}$synapses, respectively $(\mathrm{p}<0.001)$. In addition, the late component of synchronous release decayed slightly slower in $\mathrm{CPXI}^{-/-}$synapses consistent with a stronger contribution of delayed release to the release transient in these synapses. 

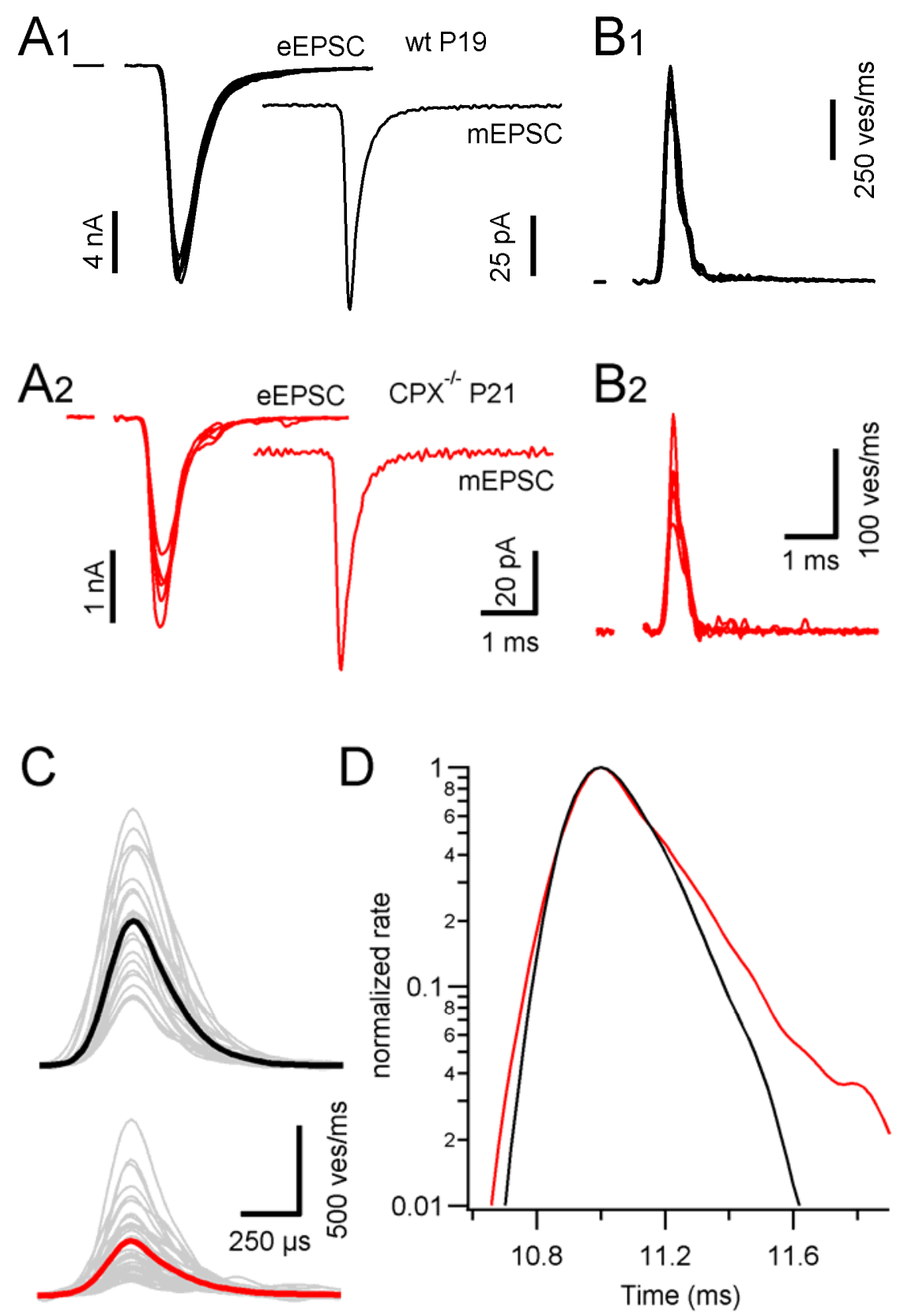

Figure 9: Similar time course of synchronous release transients underlying AP-evoked EPSCs in CPXI ${ }^{-/}$and wt mice.

Release time course was estimated by deconvolving AP-evoked EPSCs with the average mEPSC waveforms obtained from the same synapse. A, Five consecutive EPSCs $\left(\mathrm{V}_{\mathrm{h}}=-70 \mathrm{mV}\right)$ shown superimposed from a P19 $\mathrm{CPXI}^{+/+}$mouse (A1, left panel, black) and a P21 $\mathrm{CPXI}^{-/}$mouse (A2, left panel, red). Corresponding average mEPSC waveforms (right panels) are shown next to the evoked EPSCs . B, Release transients for the $\mathrm{CPXI}^{+/+}$(B1, black) and $\mathrm{CPXI}^{-/-}(\mathrm{B} 2$, red) synapse obtained by deconvolving EPSCs using a frequency-domain FFT-based deconvolution algorithm. $\mathrm{C}$, Average release transients for individual $\mathrm{CPXI}^{+/+}$(top panel, grey graces) and 
$\mathrm{CPXI}^{-/-}$synapses (bottom panel, grey graces). Thick traces represent grand averages for the entire ensembles of $\mathrm{CPXI}^{+/+}$(black) and $\mathrm{CPXI}^{-/}$(red) synapses tested. D, Comparison of the average release time course for $\mathrm{CPXI}^{-/}$(red) and wt (black) synapses. Same traces as in (C) but plotted on a semi-logarithmic scale. Both, the early rise as well as the late decay of the average release transient were slightly slower in $\mathrm{CPXI}^{-/-}$synapses.

\subsubsection{Calyceal action potential waveform and $\mathrm{Ca}^{2+}$ channel coupling}

The presynaptic AP waveform is a strong regulator of presynaptic $\mathrm{Ca}^{2+}$ influx. AP broadening can increase the number of open $\mathrm{Ca}^{2+}$ channels during the AP and prolong their open time, and thereby increase release probability (Borst and Sakmann, 1999; Sabatini and Regehr, 1997). Therefore, we tested if the calyceal AP waveform is changed in $\mathrm{CPXI}^{-/-}$synapses. Presynaptic APs were evoked by afferent fiber stimulation in brainstem slices of $\mathrm{P} 16-21 \mathrm{CPXI}^{-/-}$or wt mice, and recorded in current-clamp mode. As illustrated in Fig. 10A, the AP waveform was similar in $\mathrm{CPXI}^{-/-}(\mathrm{n}=7)$ and wt $(\mathrm{n}=8)$ mice (amplitude: $\mathrm{CPXI}^{-/-}=112.8 \pm 0.3 \mathrm{mV}$ versus $\mathrm{wt}=$ 115.2 $\pm 0.2 \mathrm{mV}$, half-width: $\mathrm{CPXI}^{-/-}=262 \pm 13 \mu$ s versus wt $=260 \pm 14 \mu \mathrm{s}$, Fig. $\left.10 \mathrm{BC}\right)$. These results indicate that changes in AP waveform are unlikely to account for the reduced synaptic strength in $\mathrm{CPXI}^{-/-}$synapses.

During postnatal maturation of the calyx synapses, a tighter spatial coupling between $\mathrm{Ca}^{2+}$ channels and docked vesicles seems to compensate for the briefer mature AP waveform causing less $\mathrm{Ca}^{2+}$ influx. Such tighter co-localization between $\mathrm{Ca}^{2+}$ channels and docked vesicles exposes the latter to higher nanodomain $\mathrm{Ca}^{2+}$ concentrations (Wang et al., 2008). We therefore hypothesized that genetic ablation of CPXI expression may interfere with this developmental refinement and result in less tight spatial coupling between $\mathrm{Ca}^{2+}$ channels and docked vesicles in $\mathrm{CPXI}^{-/-}$synapses 
which, in turn, may lower the average release probability of their docked vesicles. In order to test the hypothesis, we performed experiments to compare the effect of different concentrations of the slow $\mathrm{Ca}^{2+}$ chelator EGTA $(0.5 \mathrm{mM}$ versus $5 \mathrm{mM})$ on vesicle release elicited by AP-like presynaptic depolarizations. If VGCCs are tightly coupled to docked vesicles, even high concentrations of EGTA $(5 \mathrm{mM})$ should not be able to intercept $\mathrm{Ca}^{2+}$ ions before they reach the $\mathrm{Ca}^{2+}$ sensor of the release machinery. On the other hand, if addition of $5 \mathrm{mM}$ EGTA to the pipette solution effectively attenuates release, this would suggest that the VGCCs are physically distant from synaptic vesicles, providing sufficient time for EGTA to bind $\mathrm{Ca}^{2+}$ ions in transit (Borst and Sakmann, 1996; Meinrenken et al., 2002; Naraghi and Neher, 1997). With patch pipettes containing either 5 or $0.5 \mathrm{mM}$ EGTA, the change in membrane capacitance following a $1 \mathrm{~ms}$ step to $0 \mathrm{mV}$ was measured in $\mathrm{P} 16-26 \mathrm{CPXI}^{-/-}$and wt terminals. As shown in Fig. 10F, vesicle exocytosis was strongly attenuated by $5 \mathrm{mM}$ EGTA in $\mathrm{CPXI}^{-/-}$mice $(\mathrm{n}=12, \mathrm{p}<0.01)$ with an average capacitance jump $\Delta \mathrm{Cm} 17.3 \pm 4.8 \mathrm{fF}$ ( $\sim 216$ vesicles) but not in wt terminals ( $\Delta \mathrm{Cm}$ of $44.7 \pm 2.3 \mathrm{fF}, \mathrm{n}=15$, n.s.). This result suggests that the coupling between $\mathrm{Ca}^{2+}$ channels and docked vesicles is less tight in $\mathrm{CPXI}^{-/-}$terminals which, given the similar $I_{\mathrm{Ca}(\mathrm{V})}$ amplitudes, may lead to a lower release probability during AP-evoked release in $\mathrm{CPXI}^{-/-}$synapses. 

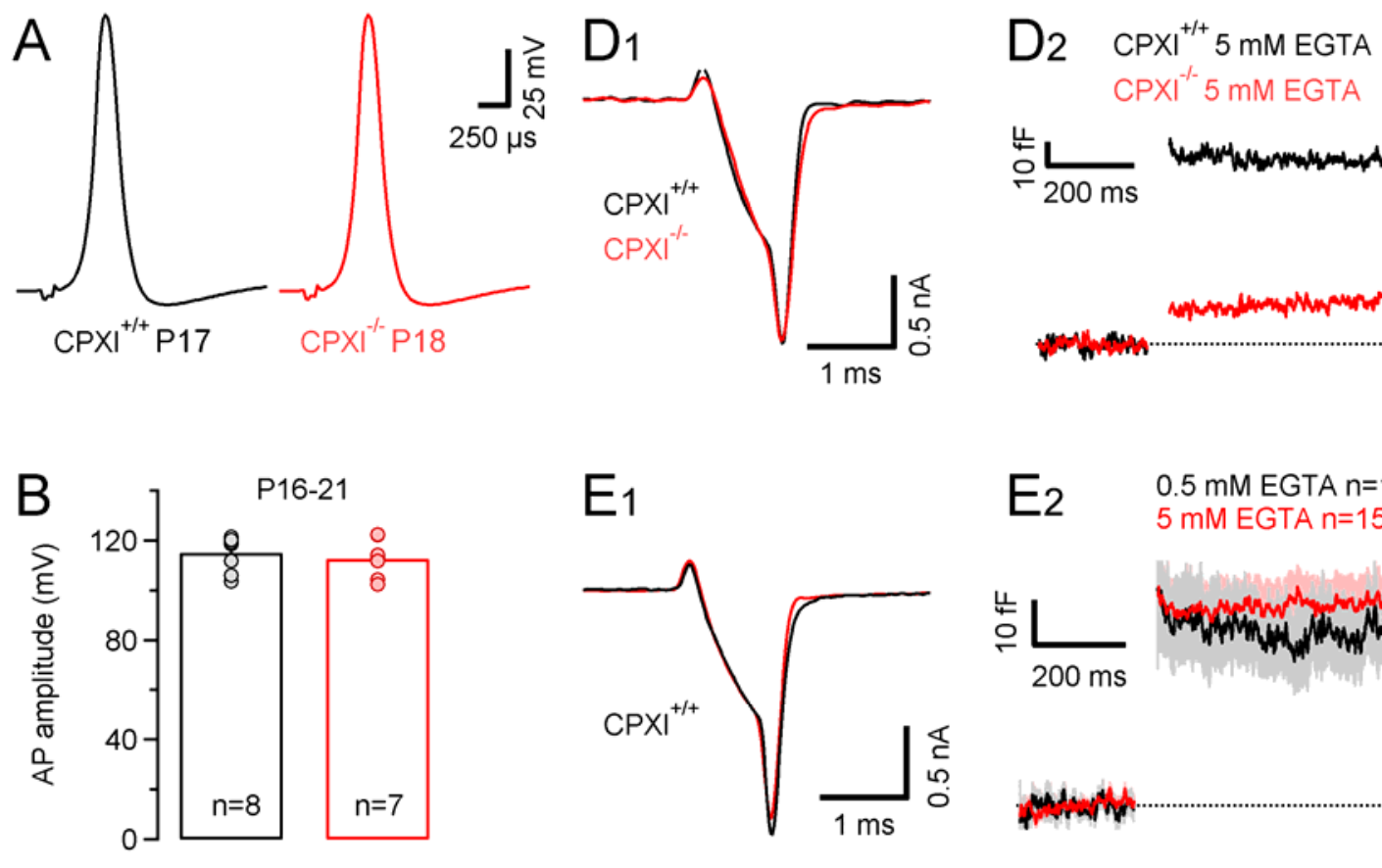

$E_{1}$

E2 $\quad \begin{array}{ll}0.5 \mathrm{mMEGTA} n=10 \\ \mathrm{mMEGTAn}=15\end{array}$
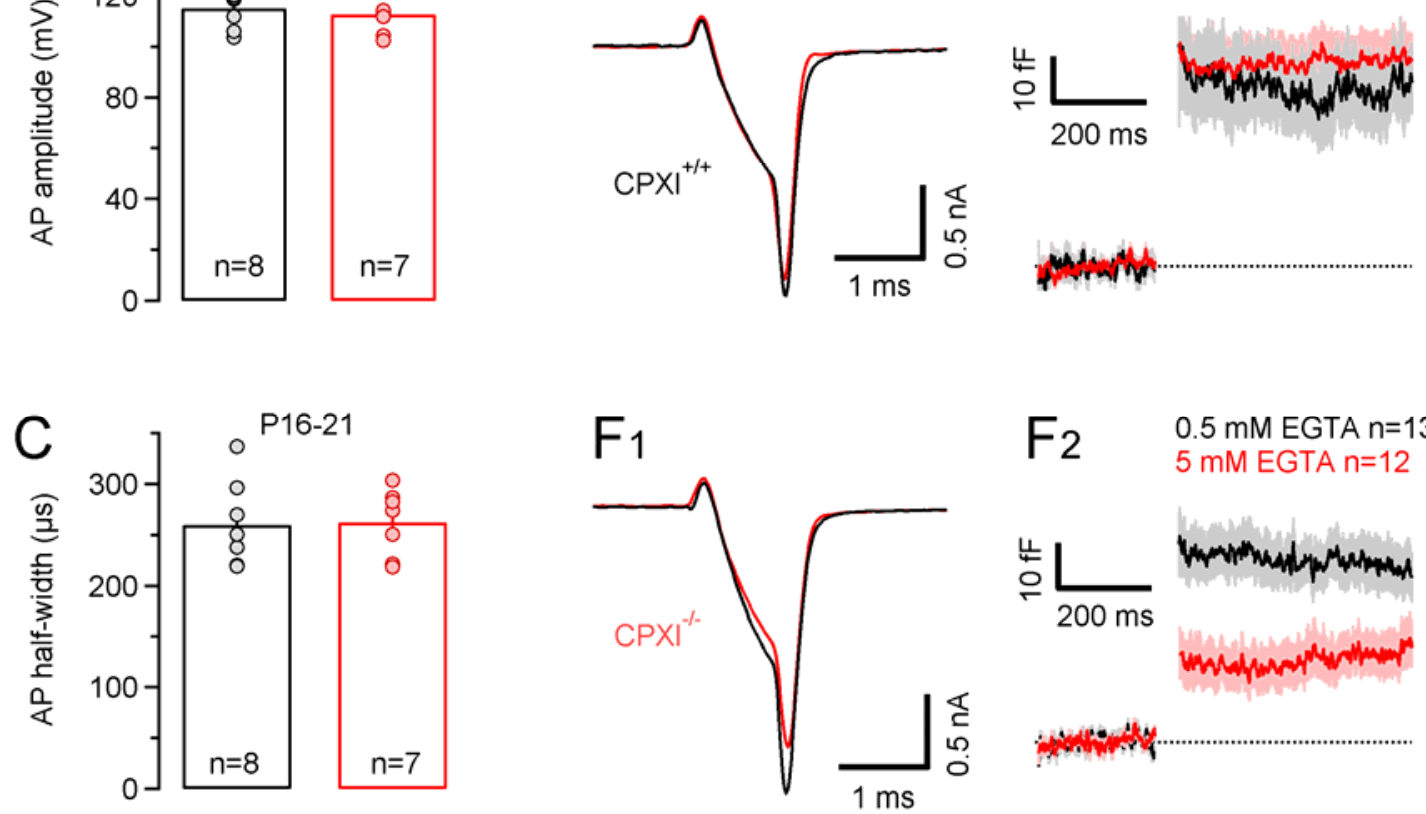

F2 $0.5 \mathrm{mM} \mathrm{EGTAn=13}$

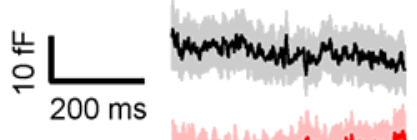

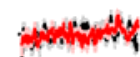

Figure10: Unaltered calyceal AP waveform but higher sensitivity of vesicle exocytosis to intracellular EGTA in $\mathrm{CPXI}^{-/-}$terminals.

A, Presynaptic calyceal APs elicited by afferent fiber stimulation and recorded in current-clamp configuration in a $\mathrm{P} 17 \mathrm{CPXI}^{+/+}$(black) and a $\mathrm{P} 18 \mathrm{CPXI}^{-/-}$(red) terminal. B,C Averaged AP amplitudes (B) and AP half-widths (C) were similar in $\mathrm{CPXI}^{+/+}$ (black) and $\mathrm{CPXI}^{-/-}$(red) terminals. Number of terminals tested as indicated. D,E,F, Presynaptic $\mathrm{Ca}^{2+}$ influx (D1,E1,F1) and vesicle exocytosis (D2,E2,F2) in response to AP-like depolarizations ( $1 \mathrm{~ms}$ steps to $0 \mathrm{mV}$ ). D, Recordings from a $\mathrm{CPXI}^{+/+}$(black) and a $\mathrm{CPXI}^{-/}$(red) terminal with $5 \mathrm{mM}$ EGTA in the pipette solution. Note the similar $I_{\mathrm{Ca}(\mathrm{V})}$ but strongly attenuated $\Delta \mathrm{C}_{\mathrm{m}}$ in the $\mathrm{CPXI}^{-/-}$terminal. E,F, Average traces for $I_{\mathrm{Ca}(\mathrm{V})}$ and $\Delta \mathrm{C}_{\mathrm{m}}$ recorded in $\mathrm{CPXI}^{+/+}(\mathbf{E})$ and $\mathrm{CPXI}^{-/-}(\mathbf{F})$ terminals with either $0.5 \mathrm{mM}$ EGTA (black traces) or $5 \mathrm{mM}$ EGTA (red traces) in the pipette solution. Light gray and light red areas in $(\mathrm{E} 2, \mathrm{~F} 2)$ represent \pm SEM. While exocytosis in $\mathrm{CPXI}^{+/+}$terminals was 
relatively insensitive to EGTA $(\mathbf{E})$, this slow $\mathrm{Ca}^{2+}$ chelator attenuated the average $\Delta \mathrm{C}_{\mathrm{m}}$ value by $>50 \%$ in $\mathrm{CPXI}^{-/-}$terminals, $\mathrm{p}<0.01(\mathbf{F})$.

\subsection{Comparison of short-term plasticity in $\mathrm{CPX}^{--}$and wt synapses}

Before hearing onset (P12-13), pronounced short-term depression is generally observed in calyx of Held synapses. Mechanisms generating stronger synaptic depression in immature synapses include higher release probability and thereby more rapid vesicle pool depletion, a stronger inactivation of presynaptic $\mathrm{Ca}^{2+}$ channels and more severe postsynaptic receptor desensitization (Borst and Soria van Hoeve, 2012). As the calyx synapses mature, the magnitude of depression decreases (Taschenberger and von Gersdorff, 2000). To study the effect of CPXI deletion on synaptic plasticity, EPSC trains evoked by afferent-fiber stimulation using different frequencies $(50,100$ and $200 \mathrm{~Hz}$ ) were recorded in $\mathrm{CPXI}^{-/-}, \mathrm{CPXI}^{+/-}$and wt synapses. Sample traces are shown in Fig. 11A. Consistent with previous reports, EPSC trains of wt synapses depressed. In contrast, $\mathrm{CPXI}^{-/-}$mice $(\mathrm{n}=50$, pooled data, P16-21) showed strong facilitation during the initial EPSCs in the trains. The maximum facilitation, which usually occurred following the second or third stimulus, was $107 \pm 3 \%, 120 \pm 4 \%$ and $140 \pm 6 \%$ of the first EPSC amplitude at the stimulus frequencies of $50 \mathrm{~Hz}, 100 \mathrm{~Hz}$ and $200 \mathrm{~Hz}$, respectively. At steady state, $\mathrm{CPXI}^{-/-}$synapses showed a reduced steady-state depression with $72.1 \pm 6 \%, 52.5 \pm 5.3 \%$ and $37.1 \pm 4.7 \%$ of the first EPSC amplitude when compared to wt mice $(25.1 \pm 1.6 \%, 17.1 \pm 1.2 \%$ and $10.1 \pm 0.7 \%$ steady-state depression, $\mathrm{n}=33$, pooled data, $\mathrm{P} 16-21)$. Interestingly, heterozygous $\mathrm{CPXI}^{+/}$mice showed a moderately reduced averaged EPSC amplitude with $-8.45 \pm 1.42 \mathrm{nA}(\mathrm{n}=11$, P16-21) but virtually unchanged synaptic plasticity with $27.9 \pm 2.8 \%, 23.0 \pm 2.2 \%$ and $14.2 \pm 1.1 \%$ depression at steady state relative to the first EPSC amplitude for $50 \mathrm{~Hz}$, $100 \mathrm{~Hz}$, and $200 \mathrm{~Hz}$ trains, respectively. Average EPSC amplitudes versus stimulus 
number are plotted in Fig. 11BC. The reduced steady-state depression and enhanced synaptic facilitation of EPSCs during trains stimulation in $\mathrm{CPXI}^{-/-}$synapses is consistent with the lower release probability in these synapses.
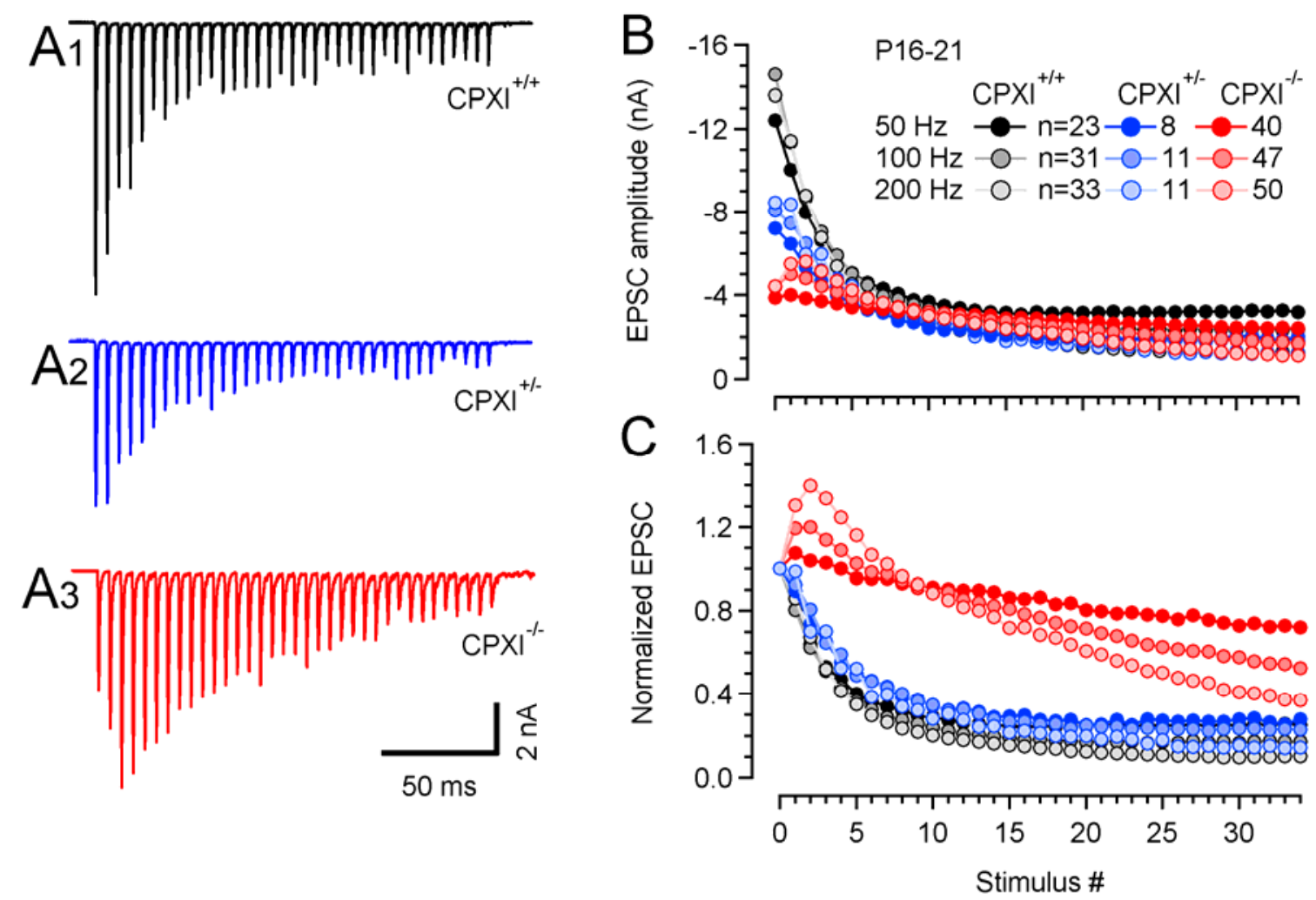

Figure11: Altered short-term plasticity in the calyx synapses of $\mathrm{CPXI}^{-/-}$mice.

A, $100 \mathrm{~Hz}$ trains of 35 EPSCs evoked by afferent-fiber stimulation in a $\mathrm{CPXI}^{+/+}$ (black), a CPXI ${ }^{+-}$(blue) and a $\mathrm{CPXI}^{-/-}$(red) calyx synapse. B,C, Pooled data of EPSC amplitudes (B) and normalized EPSCs (C) were plotted as a function of stimulus number. EPSC amplitudes were normalized to the peak of the initial EPSCs. Presynaptic AP trains consisted of 35 APs elicited at a frequency of $50 \mathrm{~Hz}, 100 \mathrm{~Hz}$ and $200 \mathrm{~Hz}$. Same color code as in (A). $\mathrm{CPXI}^{-/}$mice had reduced average EPSC amplitudes and, in contrast to $\mathrm{CPXI}^{+/+}$mice, showed synaptic facilitation for all the stimulus frequencies tested. A reduced synaptic depression is observed in heterozygous $\mathrm{CPXI}^{+/}$mice. EPSC amplitudes were obtained from averages of at least five repetitions per cell and stimulus frequency. 


\subsection{Rescue of altered EPSC amplitudes and short-term plasticity in CPX ${ }^{/-}$synapses}

In order to estimate the vesicular release probability $\left(\mathrm{P}_{\mathrm{r}}\right)$, we calculated the quantal content of single AP-evoked EPSCs in wt and $\mathrm{CPXI}^{-/-}$synapses. $\mathrm{P}_{\mathrm{r}}$ can then be obtained by simply dividing this quantity by the total number of release-ready vesicles. Because fast, synchronous AP-evoked release predominantly recruits vesicles from the fast-releasing pool (approximately $50 \%$ of the total RRP) (Sakaba, 2006), we divided our estimates for quantal content by only $50 \%$ of the total RRP as quantified by the average $\Delta \mathrm{C}_{\mathrm{m}}$ in response to a $50 \mathrm{~ms}$ depolarization. Release probability in $\mathrm{CPXI}^{-/-}$mice was strongly reduced, with an averaged value of $\mathrm{P}_{\mathrm{r}}=0.03$ compared to $\mathrm{P}_{\mathrm{r}}=0.08$ in wt mice. These values should be regarded as lower estimates because estimating quantal content by simply diving EPSC amplitude by mEPSC amplitude does not take into account the temporal jitter of quantal release. In fact, this method assumes that quanta are release in perfect synchrony, neglects late quanta and thereby underestimates quantal content. Therefore, Taschenberger et al. (2005) multiplied the ratio eEPSC/mEPSC by a factor of $\sim 1.4$ in order to correct the estimated quantal content. Applying such correction, we arrive at $\mathrm{P}_{\mathrm{r}}=0.05$ and $\mathrm{P}_{\mathrm{r}}=0.11$ for $\mathrm{CPXI}^{-/-}$and wt synapses, respectively.

In order to test whether AP-evoked EPSCs and synaptic plasticity can be rescued in $\mathrm{CPXI}^{-/-}$synapses to wt-like pattern, we raised the external $\mathrm{Ca}^{2+}$ concentration $\left(\left[\mathrm{Ca}^{2+}\right]_{\mathrm{e}}\right)$ during the recordings. We compared AP-evoked EPSCs in $200 \mathrm{~Hz}$ trains in normal external $\mathrm{Ca}^{2+}(2 \mathrm{mM})$ with those recorded after elevating $\left[\mathrm{Ca}^{2+}\right]_{\mathrm{e}}$ to $4 \mathrm{mM}$. Under the latter conditions, synaptic facilitation in $\mathrm{CPXI}^{-/-}$mice was converted to wt-like synaptic depression $(n=9)$ Fig. 12A and EPSC amplitudes were strongly augmented from an average value of $1.3 \pm 0.18 \mathrm{nA}$ to $5.1 \pm 1.01 \mathrm{nA}(\mathrm{n}=9, \mathrm{p}<0.01$, t-test $)$. Fig.12BC 
summarizes these results.
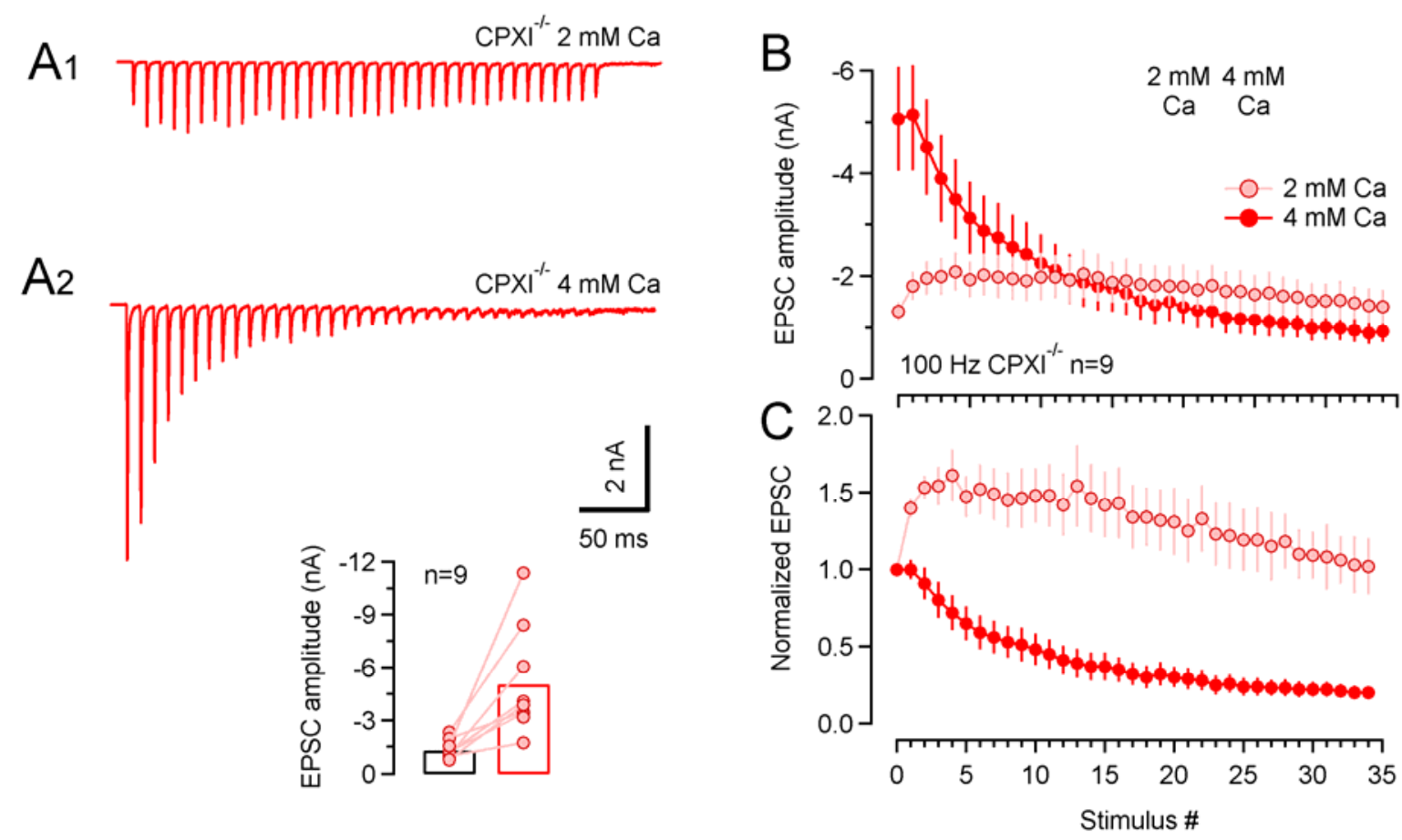

Figure 12: Altered EPSC amplitudes and short-term plasticity in $\mathrm{CPXI}^{-/}$ synapses can be largely rescued by raising extracellular $\left[\mathrm{Ca}^{2+}\right]$ to increase release probability.

A, $100 \mathrm{~Hz}$ EPSC trains recorded in a $\mathrm{CPXI}^{-/-}$synapse in normal $(\mathbf{A 1}, 2 \mathrm{mM})$ and elevated (A2, $4 \mathrm{mM})$ external $\left[\mathrm{Ca}^{2+}\right]$. B,C, Summary data obtained from a total of 9 $\mathrm{CPXI}^{-/-}$synapses showing average EPSC amplitudes (B) and normalized amplitudes (C) as a function of stimulus number for recordings in normal (2 $\mathrm{mM}$, light red) and elevated $\left(4 \mathrm{mM}\right.$, red) external $\left[\mathrm{Ca}^{2+}\right]$. EPSC amplitudes were normalized to the peak amplitudes of the initial EPSC in the trains. Inset: Changes in EPSC amplitudes $\left(1^{\text {st }}\right.$ EPSC in the trains) after switching from $2 \mathrm{mM}$ to $4 \mathrm{mM}$ external $\left[\mathrm{Ca}^{2+}\right]$. On average, EPSC peak amplitudes increased about four fold.

\subsection{Strongly enhanced asynchronous release in mature $\mathrm{CPXI}^{--}$synapses.}

Following high frequency EPSC trains, we observed an enhanced asynchronous release in mature $\mathrm{CPXI}^{-/}$calyces $(\mathrm{P} 16-21,100-300 \mathrm{~Hz}$ stimulus trains) which was absent from young homozygous $\mathrm{CPXI}^{-/-}(\mathrm{P} 8-10)$, heterozygous $\mathrm{CPXI}^{+/}$and also wt 
mice. The delayed release persisted for $\geq 500 \mathrm{~ms}$ after the end of the EPSC trains (Fig. 13A3). Asynchronous release is observed at a variety of synapses (Lu and Trussell, 2000). At the calyx of Held however, asynchronous release is usually scarce (Scheuss et al., 2007), consistent with the role of this synapse as a fast, faithful and temporal precise relay. To estimate the total asynchronous release in $\mathrm{CPXI}^{-/-}$synapses, we integrated the current after offset correction by subtracting a single or double exponential fit to the baseline. The baseline current, possibly generated by residual glutamate in the synaptic cleft, was determined by dividing the current into segments of $10 \mathrm{~ms}$ duration, determining the minimum absolute current in such segments and interconnecting the minima. Cumulative charges versus time are plotted in Fig.13B. Current integrals were converted to vesicle numbers by division by the average mEPSC charge obtained for each individual synapse (on average $-23.2 \pm 1.08 \mathrm{fC}$ in $\mathrm{CPXI}^{-/-}$mice, $\mathrm{n}=43$ ). We estimated total numbers of 305,492 and 562 asynchronously released vesicles for 100, 200 and $300 \mathrm{~Hz}$ trains, respectively.

The average ratio of asynchronous versus synchronous release during the $200 \mathrm{~Hz}$ EPSC trains was significantly higher for $\mathrm{CPXI}^{-/-}$compared to wt synapses $(36.4 \pm 2.0 \%$, $\mathrm{n}=50$ versus $4.1 \pm 0.7 \%, \mathrm{n}=32, \mathrm{p}<0.001$, t-test $)$ or $\mathrm{CPXI}^{+/-}$synapses $(2.5 \pm 1.0 \%, \mathrm{n}=$ 11). The total synchronous release was estimated by dividing cumulative EPSC trains by the average mEPSC amplitude obtained for the same synapse. As expected, asynchronous release increased with higher stimulus frequency most likely due to the increase in residual $\left[\mathrm{Ca}^{2+}\right]_{\mathrm{i}}$ (Fig. 13B). For $\mathrm{CPXI}^{-/-}$synapses, the averaged rate of asynchronous release for the initial $500 \mathrm{~ms}$ after the $200 \mathrm{~Hz}$ EPSC trains was $980 \pm 68$ vesicles/s which is much smaller than the peak release rate of synchronous release $\left(\mathrm{CPXI}^{-/}\right.$mice, averaged peak release rate: $309 \pm 36$ vesicles $\left./ \mathrm{ms}, \mathrm{n}=36\right)$. 

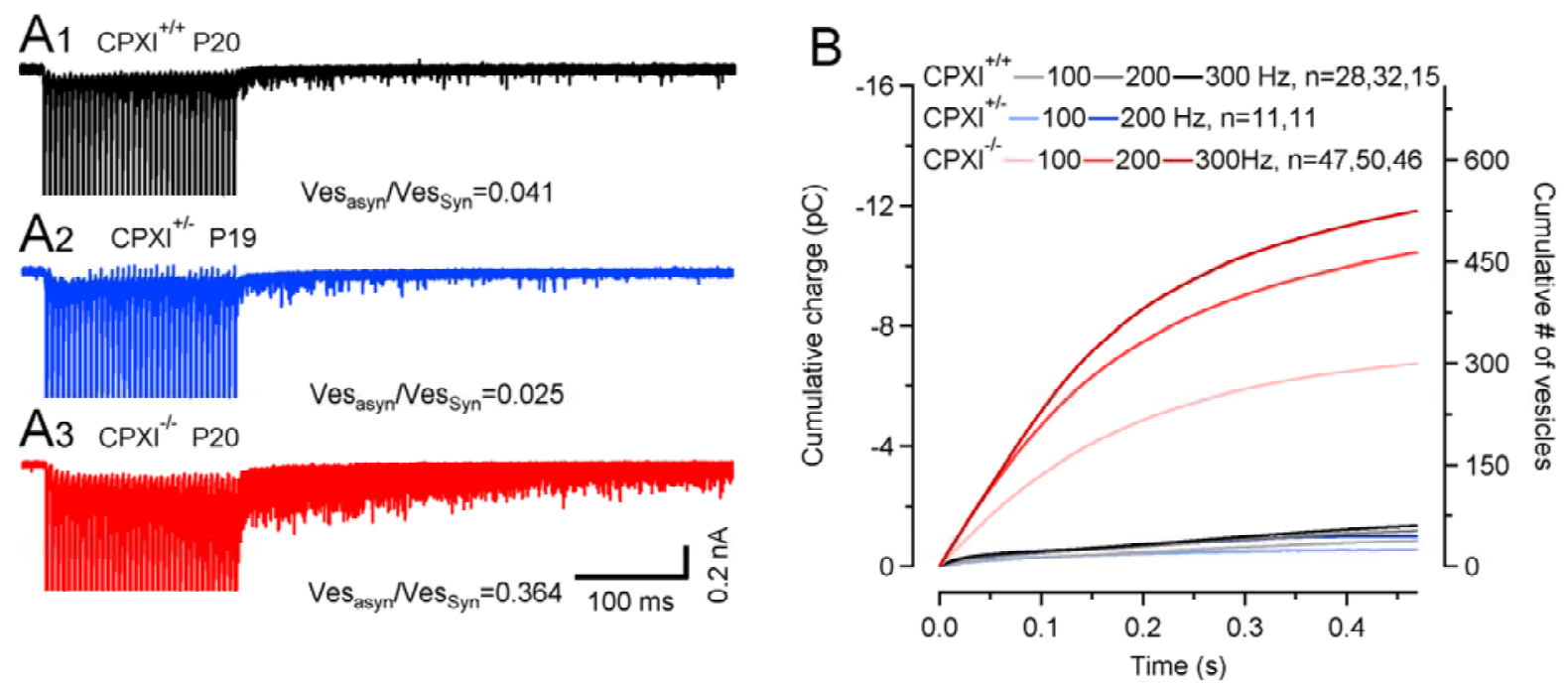

Figure 13 Strongly enhanced asynchronous release in mature $\mathrm{CPXI}^{-/}$synapses.

A, Ten consecutive traces showing $200 \mathrm{~Hz}$ trains consisting of 35 EPSCs evoked by afferent-fiber stimulation $(200 \mathrm{~Hz})$ and recorded in a P20 $\mathrm{CPXI}^{+/+}$(A1, black), a P19 $\mathrm{CPXI}^{+-}\left(\mathbf{A} 2\right.$, blue) and a P20 $\mathrm{CPXI}^{-/-}(\mathbf{A} 3$, red) calyx synapse shown superimposed. Note the strongly enhanced asynchronous release during the first $500 \mathrm{~ms}$ following the stimulus train in the synapse $\mathrm{CPXI}^{-/-}$. B, Average cumulative mEPSC charge plotted versus time after stimulation for different stimulus frequencies of $100 \mathrm{~Hz}, 200$ $\mathrm{Hz}$ and $300 \mathrm{~Hz}$ for $\mathrm{CPXI}^{+/+}$(gray and black), heterozygous $\mathrm{CPXI}^{+/-}$(blue) and $\mathrm{CPXI}^{+/+}$(red) synapses. Cumulative mEPSC charge was obtained by integration after subtracting an exponential fit to the baseline values.

\subsection{Attenuating presynaptic residual $\mathrm{Ca}^{2+}$ suppressed asynchronous vesicles fusion}

Pronounced asynchronous vesicle fusion after repetitive stimulation was also observed in presynaptic capacitance measurements. Presynaptic terminals were depolarized by 35 steps to $0 \mathrm{mV}$ (from $V_{\mathrm{h}}=-80,1 \mathrm{~ms}$ duration) at $200 \mathrm{~Hz}$. Such stimulus trains elicited a prominent change in membrane capacitance indicating $\mathrm{Ca}^{2+}$-induced vesicle fusion. In addition, there was a continuous increase in membrane capacitance in mature $\mathrm{P} 16-21 \mathrm{CPXI}^{-/-}$calyces after cessation of stimulation, 
indicating ongoing asynchronous release. Such asynchronous release was absent from presynaptic capacitance recordings in young P8-12 $\mathrm{CPXI}^{-/-}$or w.t. mice (Fig. 14). Asynchronous release is thought to require accumulation of residual $\mathrm{Ca}^{2+}$ after intense stimulation. (Barrett and Stevens, 1972; Goda and Stevens, 1994; Meiri and Rahamimoff, 1972; Miledi, 1966). $\mathrm{Ca}^{2+}$ chelators such as EGTA preferentially inhibit asynchronous release during high-frequency action-potential trains but are less effective in suppressing synchronous release (Maximov and Südhof, 2005; Otsu et al., 2004). To determine whether asynchronous release in $\mathrm{CPXI}^{-/-}$mice is triggered by elevation of intracellular $\mathrm{Ca}^{2+}$, we included a high concentration of the $\mathrm{Ca}^{2+}$ buffer EGTA $(5 \mathrm{mM})$ into the presynaptic patch pipette in order to chelate residual $\mathrm{Ca}^{2+}$ in the terminals. Under such conditions, no continuous increase in membrane capacitance after stimulus trains was observed (Fig.14A3).

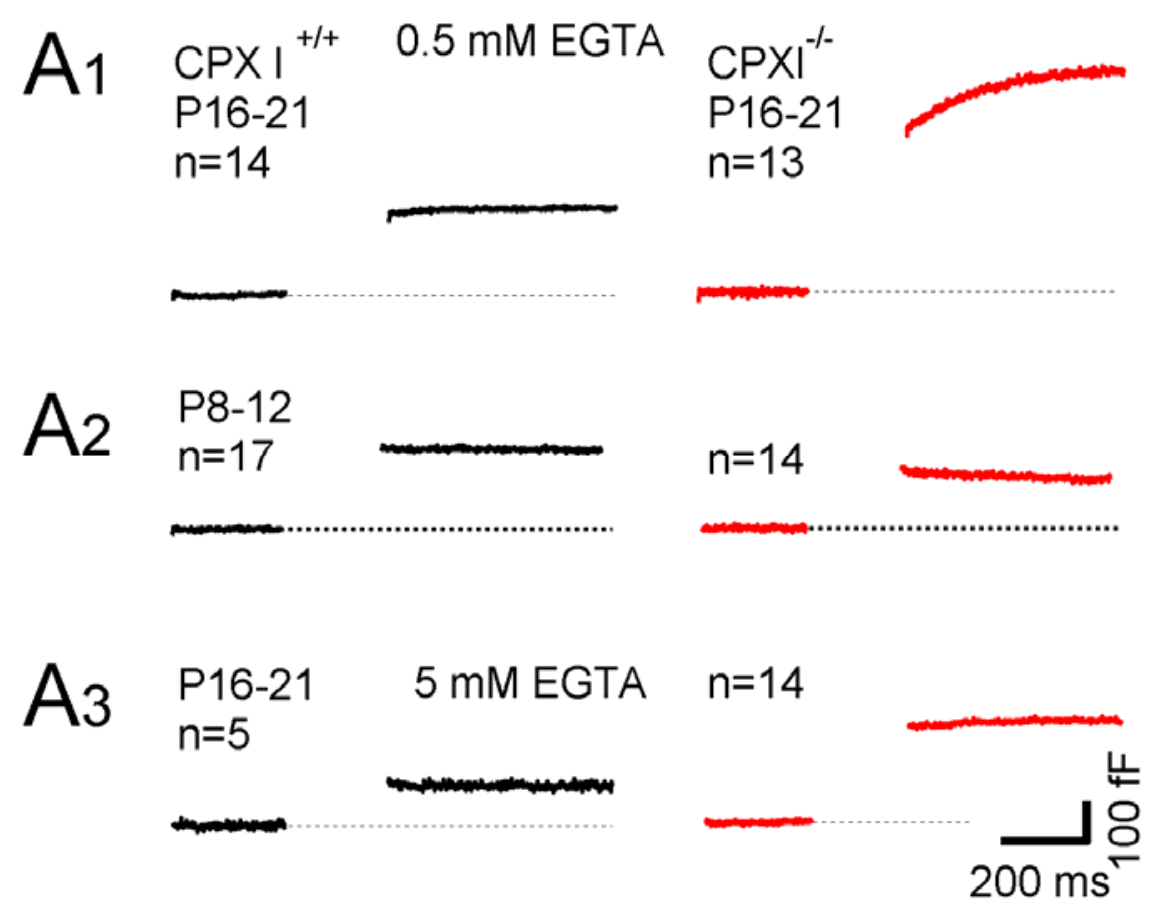

Figure14 Vesicle exocytosis after high frequency train.

A, Vesicle exocytosis after stimulus trains assayed by presynaptic membrane 
capacitance measurements. Stimulus trains consisted of thirty-five $1 \mathrm{~ms}$ steps from $\mathrm{V}_{\mathrm{h}}$ $=-80$ to $0 \mathrm{mV}$ at a frequency of $200 \mathrm{~Hz}$. Average $\Delta \mathrm{C}_{\mathrm{m}}$ traces are shown. Number of terminals tested as indicated. In the presence of $0.5 \mathrm{mM}$ EGTA (A1), $\mathrm{CPXI}^{-/-}$synapses (red) showed delayed vesicle fusion after train stimulation which was absent in $\mathrm{CPXI}^{+/+}$(black) or immature $\mathrm{CPXI}^{-/-}$mice (A2). With $5 \mathrm{mM}$ EGTA in the pipette solution, delayed release in $\mathrm{CPXI}^{-/-}$mice was suppressed (A3).

\subsection{Asynchronous release following EPSC trains in CPXI ${ }^{-\alpha}$ synapses is $\mathrm{Ca}^{2+}$ dependent}

To corroborate the finding that asynchronous release is triggered by intracellular $\mathrm{Ca}^{2+}$ as revealed by presynaptic capacitance measurements, we recorded $200 \mathrm{~Hz}$ EPSC trains ( 35 stimuli) in the absence and presence of the membrane permeable slow $\mathrm{Ca}^{2+}$ chelator EGTA-AM (tetra-acetoxymethyl ester of EGTA ) in the bath solution. As shown in Fig. 15, asynchronous release was reduced by approximately $57 \%$ after applying EGTA-AM for 8 minutes, and the maximum suppression was observed after 12 minutes application of EGTA-AM. Similar results were obtained in two other synapses. Elevating $\left[\mathrm{Ca}^{2+}\right]_{\mathrm{e}}$ from $2 \mathrm{mM}$ to $6 \mathrm{mM}$ increased not only the synchronous EPSCs but also strongly enhanced asynchronous release. Thus, asynchronous release after EPSC trains in $\mathrm{CPXI}^{-/}$synapses is not a consequence of less synchronous release because both modes of release were enhanced in the presence of high $\left[\mathrm{Ca}^{2+}\right]_{\mathrm{e}}$. 

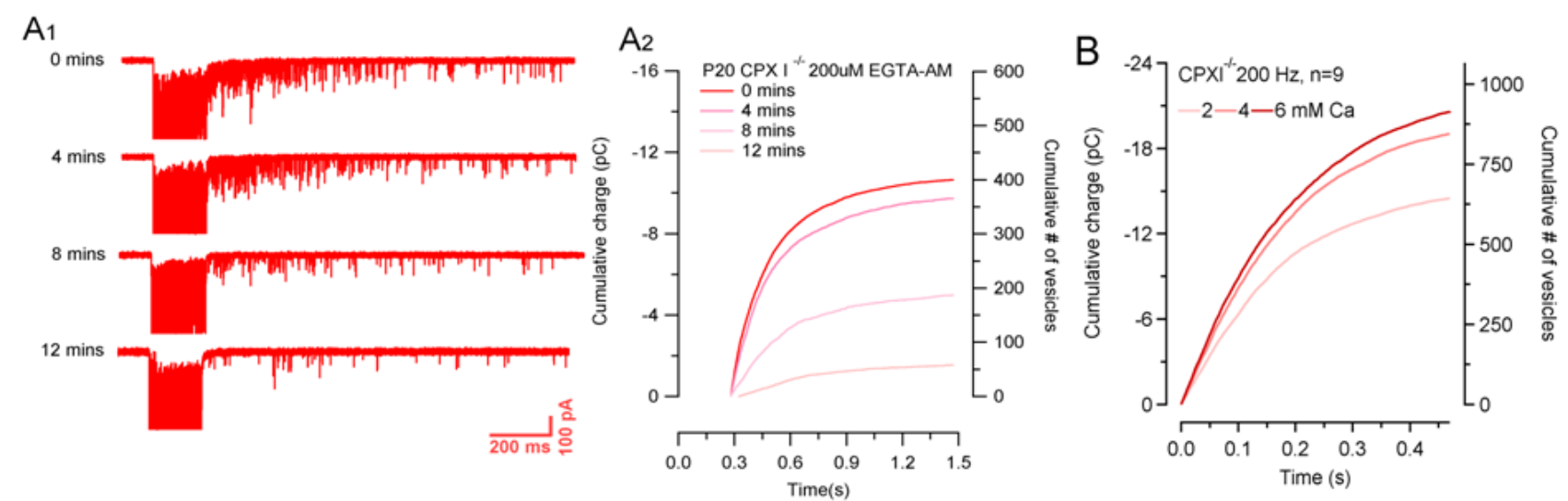

Figure 15 Effect of extracellular $\mathrm{Ca}^{2+}$ on asynchronous release.

A, Example traces showing trains of 35 EPSCs evoked by high-frequency stimulation $(200 \mathrm{~Hz})$ in $\mathrm{P} 20 \mathrm{CPXI}^{-/}$(a, red) MNTB neurons. Delayed release was recorded up to the first 1s after the stimulus train. Bath contained 200uM EGTA-AM was switched during the recording, representative traces display the asynchronous release at different time points of application (A1). Average cumulative mEPSC charge after 4 mins, 8 mins and 12 mins application of EGTA-AM are plotted versus time after stimulation in $200 \mathrm{~Hz}$ (A2). C, Average cumulative mEPSC plotted versus time for recordings in normal $(2 \mathrm{mM})$ and elevated $(4$ and $6 \mathrm{mM})$ external $\left[\mathrm{Ca}^{2+}\right]$. Elevating external $\left[\mathrm{Ca}^{2+}\right]$ augmented asynchronous release

\subsection{Correlation between asynchronous release and synchronous release}

Fig.16A shows a scatter plot of cumulative asynchronous release versus synaptic strength (peak amplitudes of the initial EPSCs in the trains) for P16-21 wt and $\mathrm{CPXI}^{-/-}$ synapses. It can be seen that peak amplitudes of AP-evoked EPSC of $w t$ and $\mathrm{CPXI}^{-/-}$ synapses overlapped to a large extend. However, the vast majority of $\mathrm{CPXI}^{-/-}$synapses showed clearly higher values of cumulative asynchronous release with most values above $-5 \mathrm{pC}$. In contrast, $w t$ synapses rarely showed cumulative asynchronous release larger than $-5 \mathrm{pC}$. Thus, there was little correlation between the degree of reduction in 
synaptic strength and the degree of enhanced asynchrounous release in individual $\mathrm{CPXI}^{-/-}$synapses. To illustrate this finding, we plotted two recordings in $\mathrm{CPXI}^{-/-}$ synapses with either strongly reduced or wt-like synaptic strength in Fig. 16B. Despite their dramatically different EPSC $_{1}$ amplitudes, both synapses showed similarly enhanced asynchronous release following the $200 \mathrm{~Hz}$ EPSC trains. Thus, it appears as if the effects of genetic ablation of $\mathrm{CPXI}^{-/-}$on synaptic strength and on asynchronous release are mediated by two distinct mechanisms.
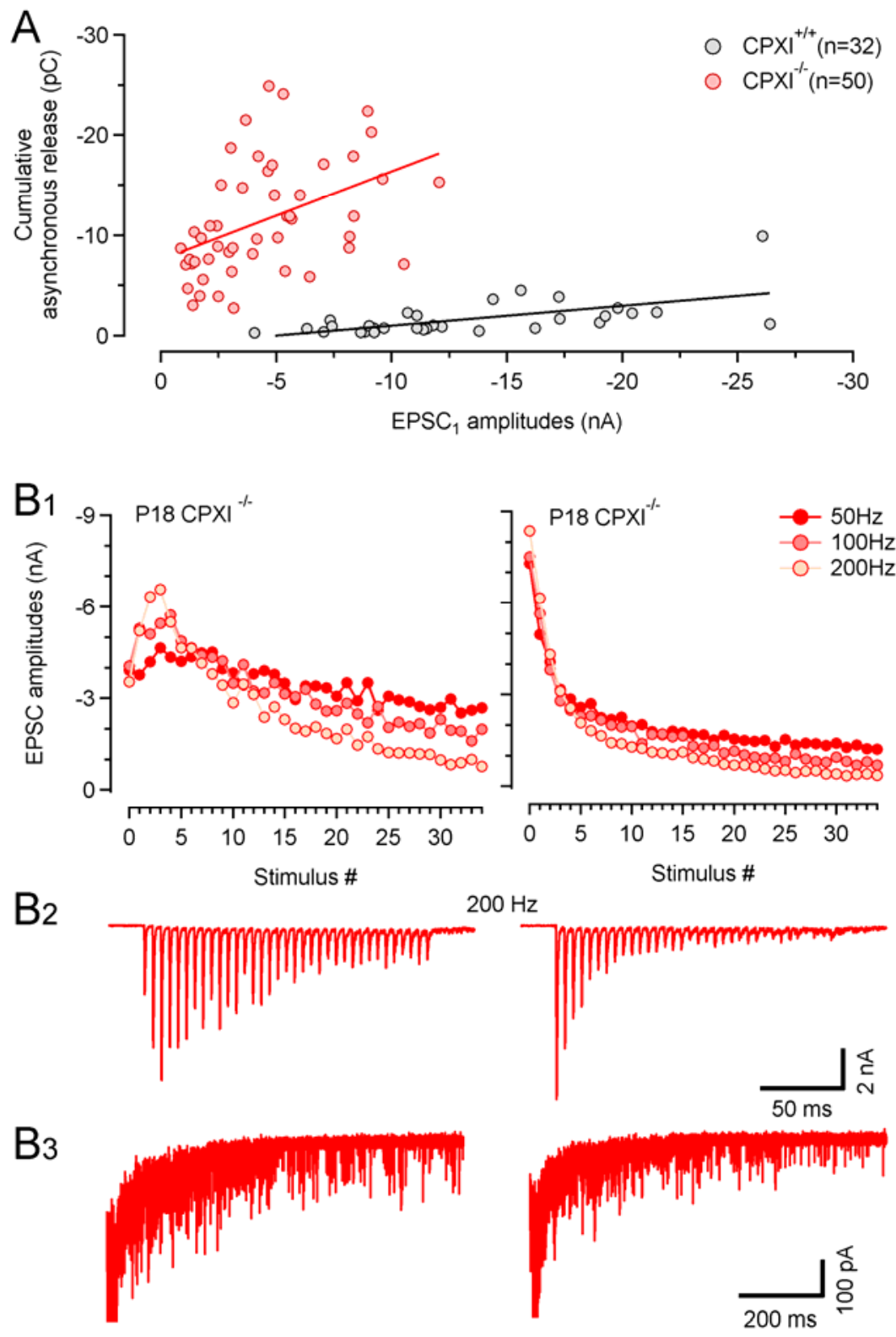
Figure 16. Correlation between amount of asynchronous release and initial EPSC amplitudes.

A, Scatter plot of cumulative asynchronous release versus initial EPSC amplitude for $200 \mathrm{~Hz}$ trains recorded in $32 \mathrm{CPXI}^{+/+}$synapses (black) and $50 \mathrm{CPXI}^{-/-}$synapses (red). note the large overlap in EPSC peak amplitudes among wt and $\mathrm{CPXI}^{-/-}$synapses. In contrast, $\mathrm{CPXI}^{-/}$synapses showed consistently higher rates of asynchrounous release after train stimulation, even if initial EPSC amplitudes were wt-like. B, Examples for two P18 $\mathrm{CPXI}^{-/-}$synapses with either low (left column) or high, wt-like synaptic strength (right column). B1, Average EPSC amplitudes obtained from 3 repetitions for 50, 100 and $200 \mathrm{~Hz}$ EPSC trains plotted as a function of stimulus number, exemplifying divergent synaptic plasticity existing in the same genotype (left: synaptic facilitation; right: synaptic depression). B2, Representative $200 \mathrm{~Hz}$ EPSC train sample traces for the same cells as shown in (B1). B3, Delayed release following $200 \mathrm{~Hz}$ EPSC trains shown at a faster time scale. Four consecutive traces are shown superimposed.

\subsection{Aberrant postsynaptic AP firing in $C P X I^{-\alpha}$ synapses}

As a fast and reliable relay synapse, the calyx of Held needs to transmit presynaptic activity over a wide range of input frequencies in order to provide well-timed inhibition to other brainstem nuclei (Borst and Soria van Hoeve, 2012). However, large amounts of asynchronous release after high-frequency trains in $\mathrm{CPXI}^{-/-}$synapses may impede faithful and temporarily precise transmission. Thus, we studied the impact of asynchronous release during physiologically relevant stimulus trains on postsynaptic AP generation in MNTB principal neurons. In current-clamp, stimulation of afferent fibers with 100 to $300 \mathrm{~Hz}$ trains consisting of 35 stimuli reliably elicited an average number of $\sim 35$ spikes in MNTB principle neurons of wt mice. In contrast, we observed a number of aberrant APs following the stimulus trains in P16-21 $\mathrm{CPXI}^{-/-}$ principal neurons (Fig. 17). The firing of aberrant APs after train stimulation was a 
robust phenomenon observed in all recordings from $\mathrm{CPXI}^{-/-}$mice. The average number of APs varied depending on stimulation frequency $(100 \mathrm{~Hz} 50.42 \pm 3.61, \mathrm{n}=17 ; 200$ $\mathrm{Hz} 49 \pm 3.07, \mathrm{n}=18 ; 300 \mathrm{~Hz} 44.27 \pm 3.67, \mathrm{n}=15)$. These observations indicate that CPXI expression is essential for faithful and precise high-frequency transmission at the calyx of Held synapse.
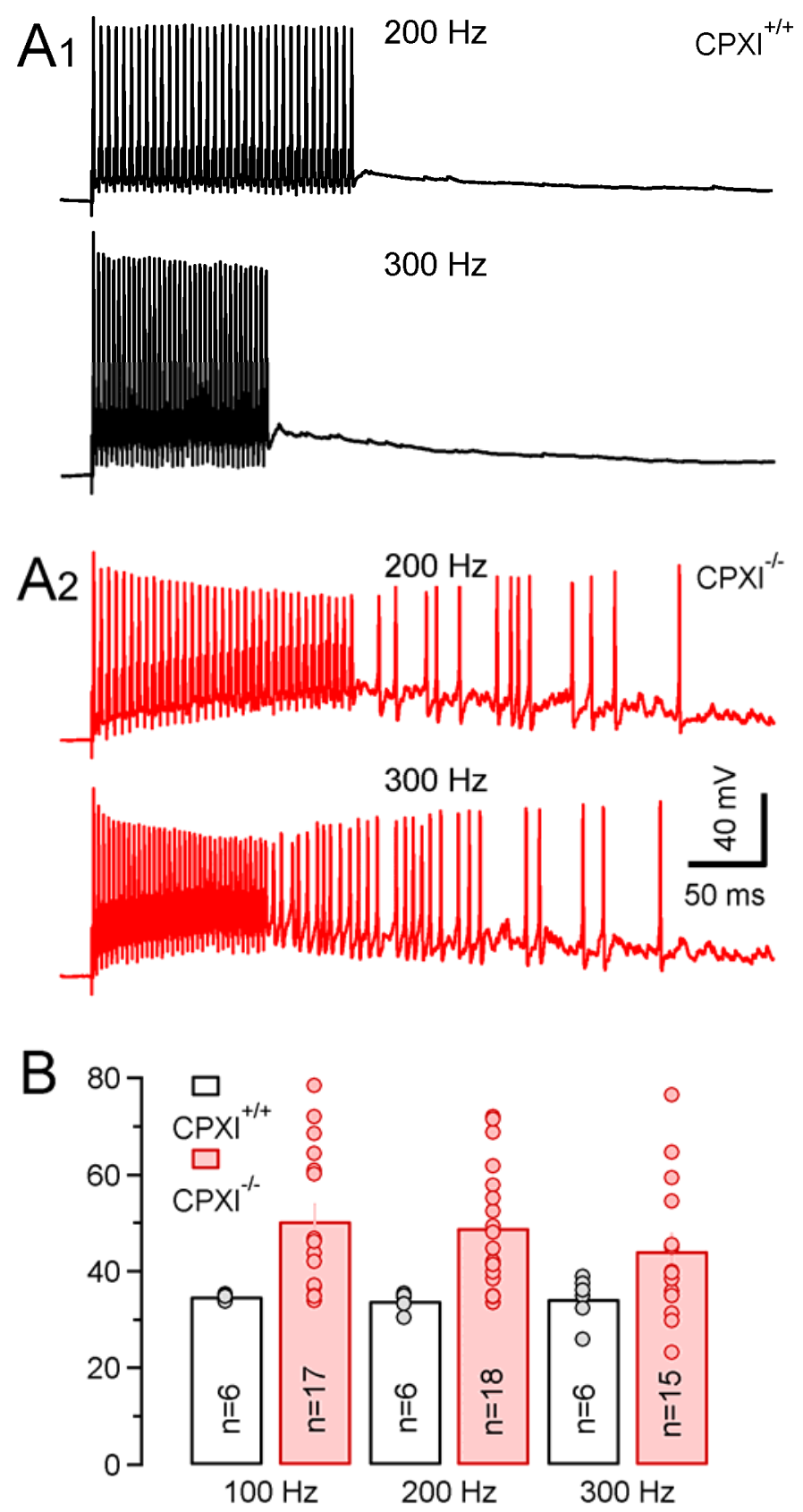
Figure 17: Asynchronous release in $\mathrm{CPXI}^{-/-}$mice promotes aberrant postsynaptic firing.

A, $200 \mathrm{~Hz}$ (top) and $300 \mathrm{~Hz}$ (bottom) AP trains elicited by afferent fiber stimulation and recorded in a $\mathrm{CPXI}^{+/+}$(A1) and a $\mathrm{CPXI}^{-/-}$(A2) principle MNTB neuron in current-clamp mode. While wt synapses generally followed presynaptic stimulation faithfully, $\mathrm{CPXI}^{-/}$neurons tended to generate numerous aberrant spikes following the stimulus trains, presumably due to a summation of asynchronously released quanta. B, Average numbers of AP elicited by high-frequency stimulus trains (100, 200 and 300 $\mathrm{Hz}, 35$ stimuli) obtained from wt (black) and $\mathrm{CPXI}^{-/-}$(red) synapses. Number of cells recorded as indicated. Average numbers of APs in $\mathrm{CPXI}^{+/+}$principal neurons were 35, 34 and 34 for 100, 200 and $300 \mathrm{~Hz}$, respectively, while $\mathrm{CPXI}^{-/-}$principal fired on average $>44$ postsynaptic APs for any of these frequencies.

\subsection{Delayed recovery of EPSCs from depression in $\mathrm{CPXI}^{\prime-}$ synapses}

As shown above, synchronous and asynchronous release components were differentially affected in CPXI-deficient calyx synapses: asynchronous release was strongly augmented while synchronous EPSCs were reduced in most $\mathrm{CPXI}^{-/-}$synapses. We therefore considered the possibility, that such divergent effects are observed because distinct vesicles pools generate asynchronous and synchronous release components at the calyx synapse. If, on the other hand, both asynchronous and synchronous release were fed by the same vesicle pool, competition should be observed. For example, (Otsu et al., 2004) successfully suppressed asynchronous release using prolonged repetitive trains of synchronous EPSCs. Assuming a replenishment rate constant of $\sim 1 / 4 \mathrm{~s}^{-1}$ (von Gersdorff et al., 1997; Weis et al., 1999), and a total number of 'fast' release sites of approximately half of the readily releasable pool estimated by capacitance measurement $(0.5 \times 4600=2300)$ (Fig. 6), the maximum rate of vesicle replenishment can be calculated by multiplying the replenishment rate constant by the number of release sites, which is 575 vesicles/s. Because the average asynchronous release rate $(980 \pm 68$ vesicles/s for $200 \mathrm{~Hz}$ trains $)$ 
is significantly higher than the maximum rate of vesicle replenishment, recovery from synaptic depression due to vesicle depletion should be delayed in $\mathrm{CPXI}^{-/-}$synapses if asynchronously and synchronously released vesicles were from the same vesicle pool. Fig. 18 shows that this is indeed the case. Using afferent fiber stimulation with 200 $\mathrm{Hz}$ trains consisting of 25 stimuli, recovery from depression was monitored by eliciting a test EPSC at different inter-stimulus intervals $(250 \mathrm{~ms}-16 \mathrm{~s})$ in P14-21 $\mathrm{CPXI}^{-/-}$and wt synapses. The $250 \mathrm{~ms}$ time interval was chosen as the shortest interval because at that time remaining $\mathrm{Ca}^{2+}$-dependent facilitation of presynaptic $I_{\mathrm{Ca}(\mathrm{V})}$ and/or postsynaptic AMPA-receptor desensitization can be expected to be very small. To calculate the recovery ratio, we normalized the test EPSC amplitude by that of the first EPSC of the conditioning trains. Fig. 18B plots the recovery ratio versus time. For $\mathrm{CPXI}^{-/-}$synapses, the curve exhibited a drop in the early phase of the recovery time course with a minimum at $1 \mathrm{~s}$ recovery interval, indicating that indeed vesicle replenishment rate was lower than the rate of asynchronous release. For intervals longer than $1 \mathrm{~s}$, the recovery time course appeared to be similar in $\mathrm{CPXI}^{-/-}$and wt synapses. We compared the time course of recovery for larger intervals (from $2 \mathrm{~s}$ to $16 \mathrm{~s}$, excluding the initial recovery from 0.25 to $1 \mathrm{~s}$ ) by fitting single exponentials to the curves. The average time constant for $\mathrm{CPXI}^{-/-}$synapses $(\tau: 4.8 \pm 0.3 \mathrm{~s}, \mathrm{n}=12)$ was similar to that in wt synapses $(\tau: 4.0 \pm 0.6 \mathrm{~s}, \mathrm{n}=17)$. Taken together these observations suggest that the loss of CPXI does not affect the replenishment rate of vesicles, but the enhanced asynchronous release in $\mathrm{CPXI}^{-/-}$mice delayed the pool replenishment suggesting that asynchronously and synchronously released vesicles competed for the same vesicle pool. 

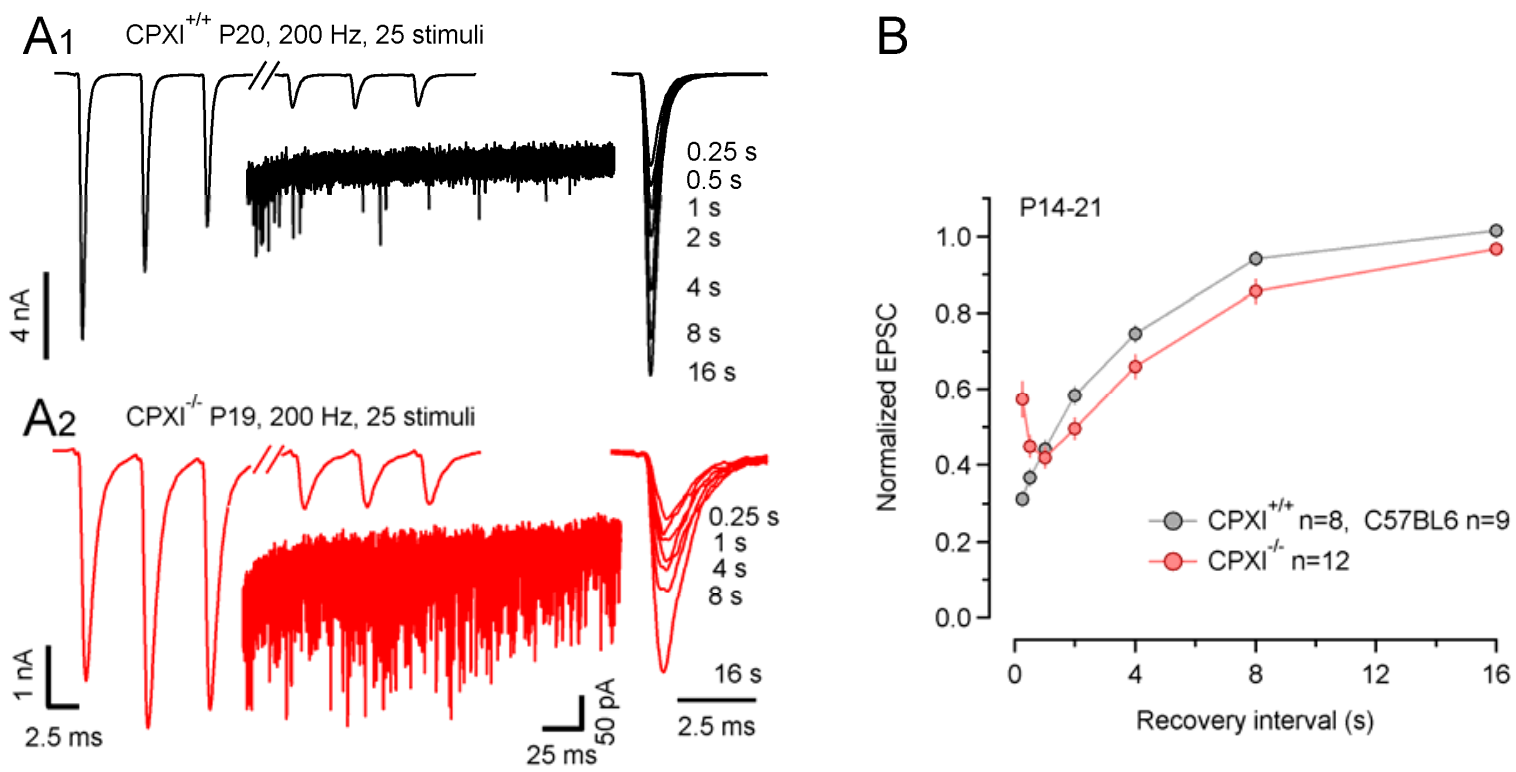

Figure18: Delayed recovery of EPSCs from depression in $\mathrm{CPXI}^{-/-}$synapses.

A, Sample traces of conditioning $200 \mathrm{~Hz}$ EPSC trains evoked by afferent-fiber stimulation (25 stimuli) that largely depleted the readily-releasable vesicle pool (left panels) recorded in a $\mathrm{P} 20 \mathrm{CPXI}^{+/+}$synapse (A1) and a $\mathrm{P} 19 \mathrm{CPXI}^{-/-}$synapse (A2). Recovery of the RRP was tested by eliciting single EPSCs after variable recovery intervals lasting from $250 \mathrm{~ms}$ to $16 \mathrm{~s}$ (right panels). Recovery intervals as indicated. Insets: comparison of asynchronous release during the first $240 \mathrm{~ms}$ immediately following the EPSC trains. B, Time course of recovery from depression after $200 \mathrm{~Hz}$ trains. Peak amplitudes of the test EPSC were normalized by diving by that of the first EPSCs in the conditioning trains. Pooled data from $7 \mathrm{CPXI}^{+/+}, 9 \mathrm{C} 57 \mathrm{BL} 6$ and 11 $\mathrm{CPXI}^{-/-}$synapses.

\subsection{Blocking asynchronous release in $\mathrm{CPXI}^{--}$terminals augments subsequent synchronous release}

In order to test whether blocking asynchronous release in $\mathrm{CPXI}^{-/-}$synapses helps replenishing the vesicle pool and thereby augments subsequent synchronous release events, we performed presynaptic capacitance measurements with either a normal $(0.5$ $\mathrm{mM})$ or a high $(5 \mathrm{mM})$ concentration of EGTA in the patch pipette (Fig. 19). 
Presynaptic terminals were stimulated with two $200 \mathrm{~Hz}$ trains consisting of $351 \mathrm{~ms}$ steps to $0 \mathrm{mV}$ (from $V_{\mathrm{h}}=-80$ ) delivered with an inter-stimulus interval of $500 \mathrm{~ms}$ $\left(\Delta \mathrm{C}_{\mathrm{m} 1}\right.$ and $\Delta \mathrm{C}_{\mathrm{m} 2}$, Fig. 19). Consistent with results described earlier, asynchronous release was effectively suppressed by $5 \mathrm{mM}$ EGTA in the pipette. Average $\Delta \mathrm{C}_{\mathrm{m}}$ traces are plotted in Fig. 19A. The second stimulus train induced a $\Delta \mathrm{C}_{\mathrm{m} 2}$ of $167 \pm 14 \mathrm{fF}(\mathrm{n}=7)$ in $\mathrm{CPXI}^{-/-}$synapses which was approximately $59 \%$ of that induced by the first stimulus train $\left(\Delta \mathrm{C}_{\mathrm{m} 1}=285 \pm 36 \mathrm{fF}\right)$ (Fig. 19A) when recording with $0.5 \mathrm{mM}$ EGTA in the pipette. Elevating the $\mathrm{Ca}^{2+}$ buffer strength by including $5 \mathrm{mM}$ EGTA in the pipette blocked the gradual increase in $\mathrm{C}_{\mathrm{m}}$ during the recovery interval presumably reflecting asynchronous release (Asyn $=129 \pm 20 \mathrm{fF}$ ) and led to an approximately $224 \pm 35 \mathrm{fF}$ larger $\Delta \mathrm{C}_{\mathrm{m} 2}$. Under these conditions, $\Delta \mathrm{C}_{\mathrm{m} 2}$ in $\mathrm{CPXI}^{-/-}$terminals was similar to that recorded in wt terminals. This result is consistent with the notion that both asynchronous synchronous release compete for the same vesicle pool.

$A_{1}$

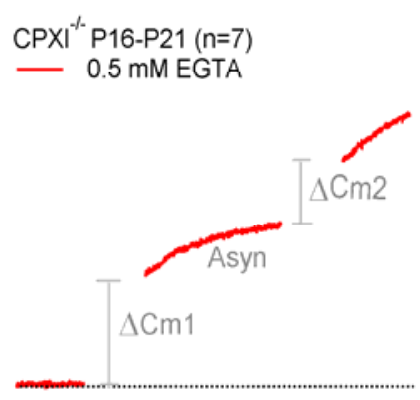

$\mathrm{A}_{2}$

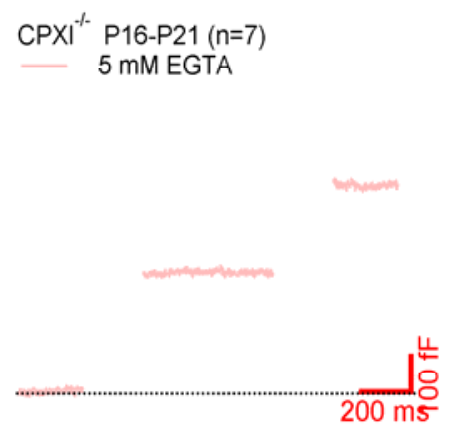

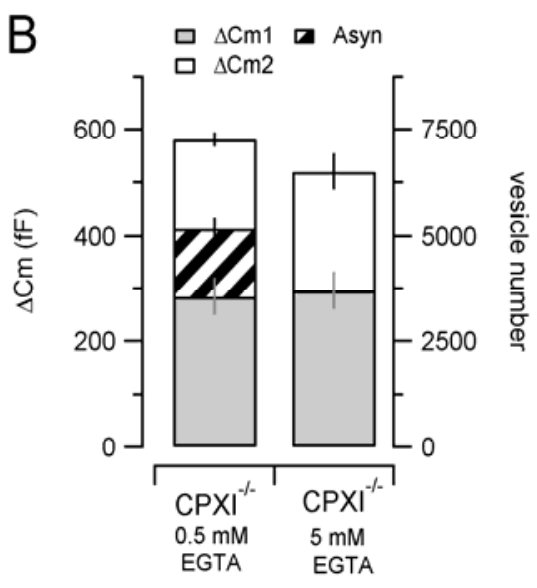

Figure 19 Restricting asynchronous release in $\mathrm{CPXI}^{-/-}$terminals augments subsequent synchronous release.

A, Vesicle exocytosis after stimulus trains assayed by presynaptic membrane capacitance measurements. Two stimulus trains consisting of $351 \mathrm{~ms}$ steps from $\mathrm{V}_{\mathrm{h}}=$ -80 to $0 \mathrm{mV}$ at a frequency of $200 \mathrm{~Hz}$ were delivered with a $500 \mathrm{~ms}$ recovery interval in between. Average $\Delta \mathrm{C}_{\mathrm{m}}$ traces are shown in $\mathrm{A} 1, \mathrm{~A} 2$. Number of terminals tested as indicated. The amount of release were quantified as indicated, and labeled as $\Delta \mathrm{C}_{\mathrm{m} 1}$ 
$\Delta \mathrm{C}_{\mathrm{m} 2}$ asynchronous release. With $0.5 \mathrm{mM}$ EGTA (A1) in the pipette solution, a gradual increase in $\mathrm{C}_{\mathrm{m}}$ was observed in $\mathrm{CPXI}^{-/-}$mice. With $5 \mathrm{mM}$ EGTA (A2) in the pipette solution, delayed release in $\mathrm{CPXI}^{-/}$mice was suppressed. B, Average capacitance changes are shown in bar graph. The amount of release during $\Delta \mathrm{C}_{\mathrm{m} 1}$, $\Delta \mathrm{C}_{\mathrm{m} 2}$ and asynchronous release were plotted in the bar graph $\left(\mathrm{CPXI}^{-/-}\right.$synapses, 0.5 mM EGTA, n=7; 5 mM EGTA, n=7).

\subsection{Down regulation of CPXII at calyx of Held synapses during development}

The absence of CPXI from calyx synapses led to functional consequences only relatively late in development $(\geq \mathrm{P} 14)$ whereas synaptic transmission in young synapses $(\leq \mathrm{P} 12)$ appeared relatively normal. Because several complexin isoforms can be co-expressed in individual neurons, other complexins may compensate for the loss of CPXI early in development when the calyx synapse has not yet fully matured. To test such a possibility, we studied CPXII expression during postnatal development of the MNTB by western blot analysis of wt mice and immunofluorescense staining of $\mathrm{CPXI}^{-/-}$mice. Since the anti-CPXI/II antibody does not distinguish between CPXI and CPXII, a specific CPXII staining of wt MNTB is impossible. Fig. 20A,B illustrate that the relative expression of CPXII is reduced from P8 to P16 and remains low thereafter. Although virtually absent from MNTB principle neurons, anti-CPXII staining was relatively abundant in the MNTB at P7 with many continuous regions that surrounded MNTB neurons and showing a morphology reminiscent of calyx terminals. Co-localization with anti-Vglut1 staining indicated expression of CPXII in calyx terminals (Fig. 20C, top row). In contrast, the immunoreactivity obtained with the CPXI/II antibody was much weaker at P21 and distributed in numerous small 
patches. No overlap with anti-Vglut1 staining was found (Fig. 20C, bottom row). Possibly, the remaining anti-CPXII-staining at this developmental stage reflects CPXII expression in small inhibitory, glycinergic boutons. Taken together, theses results suggest that the developmental loss of CPXII expression in the calyx terminal accounts, at least in part, for the observation of more severe functional deficits in older ( $\geq \mathrm{P} 16)$ calyx synapses. 

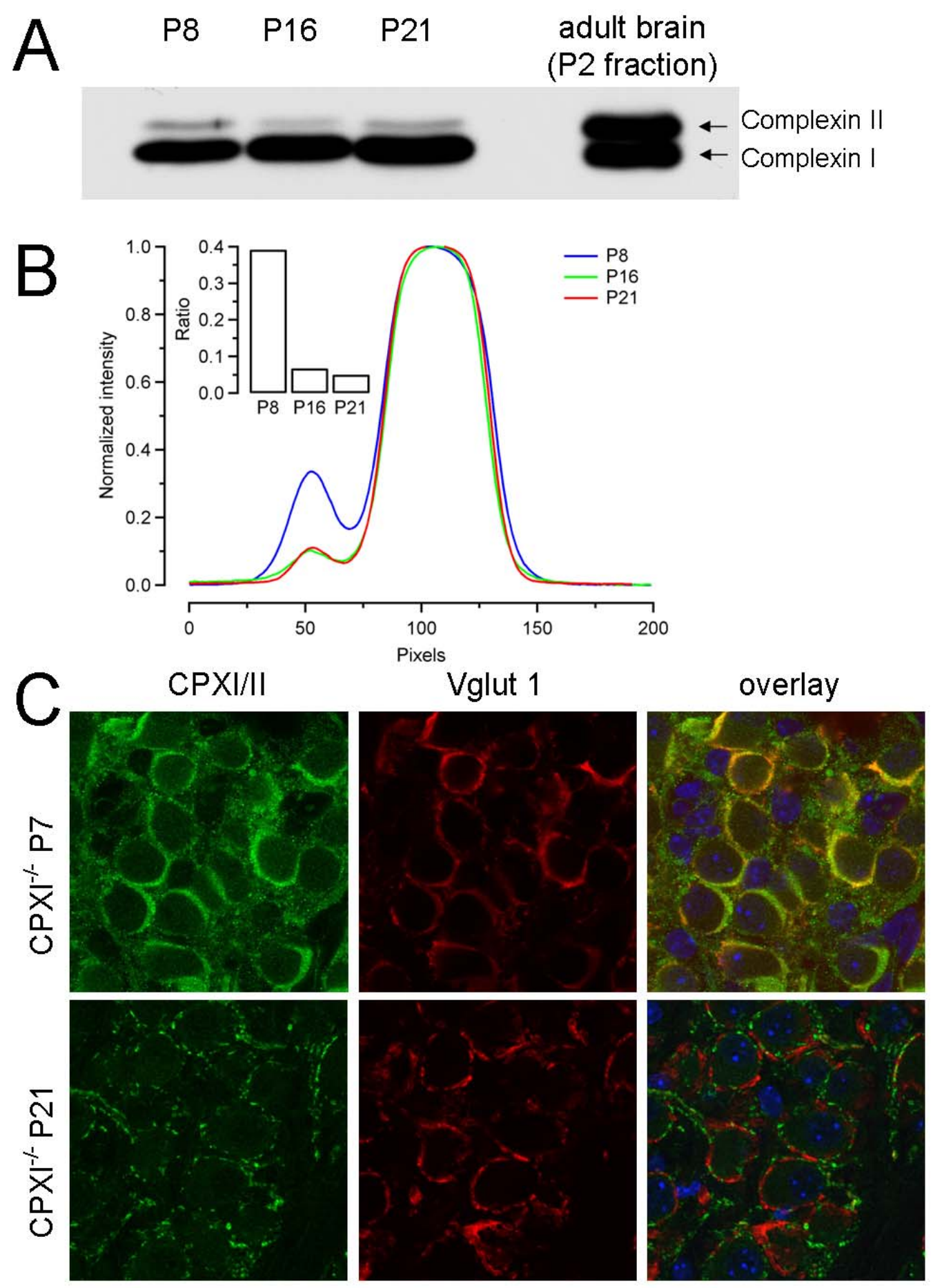

Figure 20 Down regulation of CPXII at calyx of Held synapses during development.

A, Westernblot analysis of complexin expression. Brain homogenates obtained from dissected MNTB regions of $\mathrm{P} 8, \mathrm{P} 16$ and $\mathrm{P} 21 \mathrm{CPXI}^{+/+}$mice $(10 \mu \mathrm{g}$ protein per lane $)$ were analyzed by SDS-PAGE and immunoblotting using an anti-CPXI/II antibody. 
Positions of CPXI and CPXII protein are indicated. ( $n=3$; technical replicates). B, Intensity profiles obtained from the blot shown in (A). Different exposure times were used for comparison of the ratio of CPXII/CPXI expression in order to account for the changes in absolute CPXI expression levels from P8 to P21. Inset: Expression ratio CPXII/CPXI obtained after integrating the left and the right halves of the profiles for CPXII and CPXI, respectively. $\mathbf{C}$, Immunofluorescence images representing confocal sections of MNTB regions of $\mathrm{CPXI}^{-/}$mice co-stained with an anti-CPXI/II (green) and anti-VGLUT1(red) antibody at postnatal ages P7 (left) and P21(right). Right columns show the corresponding overlays. Note the overlap of the CPXII fluorescence with that of Vglut1 at P7 (orange color) while both signals a clearly separated at P21, suggesting undetectable calyceal CPXII expression at the latter age. 


\section{Discussion}

Depending on the particular experimental conditions applied while studying vesicle release, complexins appear to exert either facilitatory or inhibitory effects on SNARE-complex-mediated vesicle exocytosis (Huntwork and Littleton, 2007; Maximov et al., 2009; Reim et al., 2001; Tang et al., 2006; Xue et al., 2007). Although the molecular mechanisms of complexin's functions is still being intensely studied, in most studies on complexin-deficient synapses fast synchronous $\mathrm{Ca}^{2+}$-triggered release generally decreased while asynchronous release was enhanced (Kaeser-Woo et al., 2012; Lin et al., 2013; Strenzke et al., 2009; Tang et al., 2006). Here, we studied the functional consequences of the absence of complexin I from calyx of Held terminals. Our results can be summarized by the following seven key observations:

(1) Deletion of CPXI led to reduced synaptic strength, decreased spontaneous release rate and enhanced asynchronous release. All of these functional defects appeared relatively late during postnatal maturation $(\geq \mathrm{P} 16)$.

(2) Even though the average rate of spontaneous release was much lower in resting CPXI-deficient synapses when compared to wt, the mEPSC frequency was strongly and long-lastingly enhanced after single AP-evoked EPSCs suggesting that newly docked vesicles were unstable and prematurely released in $\mathrm{CPXI}^{-/-}$synapses.

(3) Because of unaltered AP waveform, $\mathrm{Ca}^{2+}$ influx, RRP, and quantal size, we concluded that the reduced synaptic strength in $\mathrm{CPXI}^{-/}$-deficient synapses was caused by decreased release probability due to a changed $\mathrm{Ca}^{2+}$ sensitivity of the release machinery or altered spatial coupling between docked vesicles and VGCCs.

(4) The higher sensitivity of $\mathrm{Ca}^{2+}$-triggered vesicle release to EGTA is consistent with 
a defect in the developmental tightening of the spatial coupling between VGCCs and docked vesicles in $\mathrm{CPXI}^{-/-}$-deficient terminals.

(5) Strongly enhanced asynchronous release in $\mathrm{CPXI}^{-/-}$-deficient synapses was triggered by residual $\mathrm{Ca}^{2+}$ after high-frequency EPSC trains.

(6) Asynchronously released quanta competed with synchronously released ones for the same pool of readily releasable vesicles.

(7) EPSPs generated by asynchronous release triggered aberrant APs in MNTB principal neurons, greatly reducing the fidelity of transmission at the calyx of Held synapse.

\subsection{Multiple roles of CPXI in regulating vesicle exocytosis}

It has been clearly shown that complexin facilitates $\mathrm{Ca}^{2+}$ triggered synchronous release (Xue et al., 2007; Yang et al., 2010a). However, not all experimental results are consistent with a general role of complexins as a fusion clamp. For example, a number of preparations from complexin-deficient mice including our own study did not find an increased miniature EPSC rate (Reim et al., 2001; Strenzke et al., 2009; Xue et al., 2007). In contrast, other experimental results support an inhibitory function of complexin because an increased rate of spontaneous release was found for example in mouse cortical cultures after shRNA-mediated complexin knock-down (Maximov et al., 2009) and also in the artificial flipped SNARE cell fusion system (Giraudo et al., 2006).

Here we report that both synaptic strength as well as spontaneous release are reduced in CPXI-deficient calyces of Held indicating that CPXI is a positive modulator of synaptic transmission at this synapse. On the other hand, spontaneous release was augmented for several hundreds of $\mathrm{ms}$ after a single AP-evoked EPSC and 
asynchronous release was strongly enhanced after high-frequency EPSC trains. Both of these observations are consistent with a role of CPXI as a negative regulator or fusion clamp. Complexins are not the only presynaptic proteins that have simultaneously been attributed positive as well as negative regulatory functions. For example synaptotagmin knock-out studies revealed, that synaptotagmin plays a certain role in facilitating exocytosis, and simultaneously might serve as a clamp to prevent asynchronous and spontaneous release (Kochubey and Schneggenburger, 2011; Maximov and Südhof, 2005; Pang et al., 2011; Pang et al., 2006). Thus, it appears that certain presynaptic proteins may exert both inhibitory as well as stimulatory functions to flexibly regulate vesicle exocytosis. Especially while a vesicle is being primed for fast release, spontaneous release must be suppressed to prevent premature depletion of the primed vesicle pool. For this reason, it becomes extremely difficult for the priming machinery to prepare vesicles to be released in a fraction of a $\mathrm{ms}$ in response to the presynaptic $\mathrm{Ca}^{2+}$ influx and at the same time prevent premature fusion (Brose, 2008; Sorensen, 2009; Sudhof and Rothman, 2009). Complexin and synaptotagmin are proteins that have been demonstrated to act as key regulators with bilateral roles in vesicle exocytosis. (Tang et al.) suggested that complexins bind to partially-assembled SNARE complexes during priming, forcing completion of SNARE-complex assembly from a loose to a tightly assembled state that serves as essential substrate for the subsequent $\mathrm{Ca}^{2+}$ bound-synaptotagmin. However, if complexin acts as a fusion clamp that is removed in a later step by synaptotagmin, then the absence of synaptotagmin should leave the vesicles in a clamped state. The opposite was however observed in synatotagmin-deficient synapses where the deletion of synaptotagmin led to a dramatically enhanced spontaneous release (Kochubey and Schneggenburger, 2011; Pang et al., 2006). This suggests that complexin is not in an upstream-and-downstream relationship with 
synaptotagmin.

Complexin is most likely a multifunctional protein and this leads to divergent functional defects when studying different modes of neurotransmitter release in complexin-deficient synapses. For example, (Xue et al.) showed that the first 26 residues of complexin I facilitate vesicle exocytosis in the absence of synaptotagmin-1 (Syt1). This reaction might be mediated by an interaction with other proteins, CPXI itself to release the inhibition effect of its $\alpha$ accessory domain or phospholipids of the membrane (Dai et al., 2007). Second, the accessory $\alpha$ helix domain of itself which inserts into nascent SNARE complexes thereby prevents the completion of SNARE complex assembly (Yang et al., 2010a). Third, (Iyer et al.) demonstrated that complexin carrying the $\mathrm{cpx}^{1257}$ mutation selectively failed to clamp spontaneous release but this mutation had no influence on the amplitude of evoked EPSCs. These results, taken together, suggest that the mechanism of maintaining primed vesicles in metastable state by complexins is unimportant to control vesicle exocytosis (Xue et al., 2009; Xue et al., 2007).

\subsection{More than one mechanism for neurotransmitter release}

Distinct effects of synaptic proteins deficient on synchronous and asynchronous release have been interpreted in terms of different properties of the release machineries (Lou et al., 2005; Sun et al., 2007). Our study provides some evidence that synchronous and asynchronous release are operated by distinct mechanisms. Firstly, the suppression of synchronous release but augmentation of asynchronous release after removal of CPXI shows that they are differently modulated. Secondly, in recordings from $\mathrm{CPXI}^{+/-}$mice, the single allele deletion of CPXI impaired 
synchronous release but left asynchronous release largely unaffected and wt-like (Fig. 13). This observation suggests that the reduced expression of CPXI driven by a single allele was sufficient to clamp vesicles and largely prevent asynchronous release. But the expression level was insufficient to maintain synaptic strength during AP-evoked EPSCs. Thirdly, individual synapses from the same $\mathrm{CPXI}^{-/}$animal showed strongly divergent phenotypes regarding synaptic strength and plasticity but similarly high rates of asynchronous release following EPSC trains (Fig. 16), suggesting that genetic ablation of CPXI affects asynchronous release by a different mechanism than synchronous release.

A dual $\mathrm{Ca}^{2+}$ sensor model has been proposed by Sun et al. (2007), who postulated that the $\mathrm{Ca}^{2+}$ sensors for synchronous and asynchronous release operate in competition with each other. Deletion of synaptotagmin-2 at calyx of Held synapse revealed that a near linearly-operating $\mathrm{Ca}^{2+}$ sensor remained for vesicle exocytosis (Kochubey and Schneggenburger, 2011). One candidate for such a secondary sensor is Doc2, which participates in asynchronous and spontaneous release (Pang et al., 2011; Yao et al., 2011). Yao, Gaffaney et al. (2011) showed that asynchronous release was largely reduced when knocking down the expression of Doc2b in Synaptotagmin 1-deficient hippocampal neurons. However, a dual $\mathrm{Ca}^{2+}$ sensors model does not provide a full explanation for the reduction of spontaneous release in $\mathrm{CPXI}^{-/-}$mice in our study.

On the other hand, Neher (2010) proposed that the complexity of the presynaptic $\mathrm{Ca}^{2+}$ signal alone is sufficient to account for different components of release and no 'special' or distinct mechanisms need to be postulated for generating the kinetic features of asynchronous release. Variability between synapses in the degree of asynchronous release may simply reflect difference in $\mathrm{Ca}^{2+}$ signaling. Thus, the differences observed here between $\mathrm{CPXI}^{-/-}$and wt synapses may either be caused by differences in their $\left[\mathrm{Ca}^{2+}\right]_{\mathrm{i}}$ handling and/or by a different sensitivity of the release 
machinery to $\mathrm{Ca}^{2+}$. It is therefore desirable to measure the $\mathrm{Ca}^{2+}$ sensitivity of release in $\mathrm{CPXI}^{-/-}$synapses for example by $\mathrm{Ca}^{2+}$ uncaging via flash photolysis (Kochubey et al., 2011). Such experiments will be challenging because of the late developmental onset of the functional defects in CPXI-deficient calyx synapses.

\subsection{A possible defect in the coupling between VGCCs and docked vesicles}

In order to elucidate the mechanisms accounting for the attenuation of synchronous release in $\mathrm{CPXI}^{-/-}$mice, we measured the action potential waveform (AP), $\mathrm{Ca}^{2+}$ influx, RRP and quantal size and found all of these quantities unaltered when compared to wt synapses. However, presynaptic capacitance measurements revealed a higher sensitivity to the slow $\mathrm{Ca}^{2+}$ chelator EGTA in CPXI-deficient terminals (Fig. 10). This result is consistent with a less tight coupling between docked synaptic vesicles and VGCCs in these synapses. Rapid neurotransmitter release triggered by fast APs requires an exquisite organization of the active zone that allows interactions between

$\mathrm{Ca}^{2+}$ channels and readily releasable vesicles at the shortest possible distances. Properly positioning synaptic vesicles to VGCCs becomes an important requirement especially in mature calyx terminals that are characterized by very brief presynaptic APs (Young Jr and Neher, 2009). The distance between VGCCs and docked vesicles determines the level of micro- or nanodomain $\left[\mathrm{Ca}^{2+}\right]_{\mathrm{i}}$ sensed by the release machinery and thereby critically determines release probability (Neher and Sakaba, 2008). The presynaptic Rab-3 interacting molecule (RIM) has been reported to play an important role in maintaining a high $\mathrm{Ca}^{2+}$ channel density at calyx of Held synapses (Han et al., 2011). Furthermore, septin, a filamentous protein, has also been demonstrated to regulate the distance between VGCCs and docked vesicles during maturation of the calyx terminal, possibly participating in the developmental switch from $\mathrm{Ca}^{2+}$ 
microdomain to nanodomain signaling in the mature calyx (Yang et al., 2010b). A larger distance between docked vesicles and VGCCs is expected to lower the peak release rates and broaden the release transients underlying AP-evoked EPSCs. Indeed, deconvolution of EPSCs recorded in $\mathrm{CPXI}^{-/-}$calyx synapses revealed a strongly reduced peak release rate and additionally as slightly slower rise and decay of the release function (Fig. 9). Although complexins are not known to directly interact with $\mathrm{Ca}^{2+}$ channels, it is possible that they assistance in the process of vesicle positioning via interaction with other proteins at the active zone (Young Jr and Neher, 2009).

\subsection{The role of postsynaptic CPXI}

So far, most studies on the function of CPXI have focused on presynaptic aspects, but its postsynaptic function remains obscure. (Ahmad et al.) report a lack of postsynaptic effects following CPXI kock-down in hippocampal CA1 pyramidal cells. Neither the ratio of AMPA/NMDA receptors nor the composition of AMPA or NMDA receptors were changed. Nevertheless, we found that CPXI is strongly expressed in MNTB principle neurons and ask whether deletion of CPXI may alter synaptic transmission at calyx of Held synapses via postsynaptic mechanisms such as changes in quantal size or relative expression ratio of synaptic NMDA and AMPA channels. As shown in Fig. 8, quantal size and time course of mEPSCs were unchanged suggesting similar subunit composition of synaptic AMPARs in $\mathrm{CPXI}^{-/-}$and wt synapses. In addition, we measured the ratio of NMDAR-mediated EPSCs to AMPAR-mediated EPSCs which was unaltered in $\mathrm{CPX}^{-/-}$synapses. In both $\mathrm{CPX}^{-/-}$as well as wt mice, NMDARs contributed $\leq 3 \%$ to the EPSC amplitude. Thus, the lack of CPXI expression in MNTB principle neurons does not seem to affect the expression of postsynaptic glutamate receptor channels. 


\section{Summary}

Complexins are small synaptic proteins which cooperate with the SNARE-complex in synaptic transmission. Different roles of complexins in the regulation of vesicle exocytosis have been proposed. Based on the results of genetic mutation or knock-down or knock-out studies, it is generally agreed that complexins are involved in vesicle priming and exocytosis for fast synchronous release and in clamping vesicles to prevent asynchronous release. However, depending on cell type, organism and experimental approach used, complexins appear to either facilitate or inhibit vesicle fusion.

Here, we study the function of complexin I at the mouse calyx of Held synapse. By taking advantage of the large size of the calyx terminal, allowing direct patch-clamp recordings, we investigate the consequences of the loss of function of complexin I. We demonstrate a developmentally aggravating phenotype of reduced EPSC amplitudes and enhanced asynchronous release. Because action potential waveform, $\mathrm{Ca}^{2+}$ influx, readily releasable pool, and quantal size were all unaltered, we concluded that the reduced synaptic strength in complexin I-deficient synapses was caused by decreased vesicle release probability by either a changed $\mathrm{Ca}^{2+}$ sensitivity of the release machinery and/or a changed coupling between $\mathrm{Ca}^{2+}$ channels and docked vesicles. The strongly enhanced asynchronous release in complexin I-deficient calyx synapses triggered aberrant action potentials in MNTB principal neurons, and slowed-down the recovery of action potential-evoked EPSCs after depleting stimulus trains. Restricting asynchronous release augmented subsequent synchronous release, suggesting that synchronous and asynchronous release competed for a common pool of vesicles. 


\section{References}

Ahmad, M., Polepalli, Jai S., Goswami, D., Yang, X., Kaeser-Woo, Yea J., Südhof, Thomas C., and Malenka, Robert C. (2012). Postsynaptic Complexin Controls AMPA Receptor Exocytosis during LTP. Neuron 73, 260-267.

Angleson, J. K., and Betz, W. J. (2001). Intraterminal Ca2+ and spontaneous transmitter release at the frog neuromuscular junction. Journal of Neurophysiology 85, 287-294.

Barrett, E. F., and Stevens, C. F. (1972). The kinetics of transmitter release at the frog neuromuscular junction. J Physiol 227, 691-708.

Bellingham, M. C., Lim, R., and Walmsley, B. (1998). Developmental changes in EPSC quantal size and quantal content at a central glutamatergic synapse in rat. J Physiol 511 ( Pt 3), 861-9.

Bergsman, J. B., De Camilli, P., and McCormick, D. A. (2004). Multiple large inputs to principal cells in the mouse medial nucleus of the trapezoid body. $J$ Neurophysiol 92, 545-52.

Bollmann, J. H., Sakmann, B., and Borst, J. G. (2000). Calcium sensitivity of glutamate release in a calyx-type terminal. Science 289, 953-7.

Borst, J. G., Helmchen, F., and Sakmann, B. (1995). Pre- and postsynaptic whole-cell recordings in the medial nucleus of the trapezoid body of the rat. $J$ Physiol $\mathbf{4 8 9}$ ( Pt 3), 825-40.

Borst, J. G., and Sakmann, B. (1996). Calcium influx and transmitter release in a fast CNS synapse. Nature 383, 431-4.

Borst, J. G., and Sakmann, B. (1999). Effect of changes in action potential shape on calcium currents and transmitter release in a calyx-type synapse of the rat auditory brainstem. Philos Trans $R$ Soc Lond B Biol Sci 354, 347-55.

Borst, J. G., and Soria van Hoeve, J. (2012). The calyx of held synapse: from model synapse to auditory relay. Annu Rev Physiol 74, 199-224.

Brose, N. (2008). For Better or for Worse: Complexins Regulate SNARE Function and Vesicle Fusion. Traffic 9, 1403-1413.

Dai, H., Shen, N., Araç, D., and Rizo, J. (2007). A Quaternary SNARE-Synaptotagmin-Ca2+-Phospholipid Complex in Neurotransmitter Release. Journal of Molecular Biology 367, 848-863.

Diamond, J. S., and Jahr, C. E. (1995). Asynchronous release of synaptic vesicles determines the time course of the AMPA receptor-mediated EPSC. Neuron 15, 1097-1107.

Dittman, J. S., and Regehr, W. G. (1996). Contributions of calcium-dependent and 
calcium-independent mechanisms to presynaptic inhibition at a cerebellar synapse. Journal of Neuroscience 16, 1623-1633.

Forsythe, I. D. (1994). Direct patch recording from identified presynaptic terminals mediating glutamatergic EPSCs in the rat CNS, in vitro. Journal of Physiology 479, 381-387.

Freeman, W., and Jennifer Morton, A. (2004). Differential messenger RNA expression of complexins in mouse brain. Brain Research Bulletin 63, 33-44.

Giraudo, C. G., Eng, W. S., Melia, T. J., and Rothman, J. E. (2006). A clamping mechanism involved in SNARE-dependent exocytosis. Science 313, 676-80.

Goda, Y., and Stevens, C. F. (1994). Two components of transmitter release at a central synapse. Proceedings of the National Academy of Sciences 91, 12942-12946.

Groffen, A. J., Martens, S., Arazola, R. D., Cornelisse, L. N., Lozovaya, N., de Jong, A. P. H., Goriounova, N. A., Habets, R. L. P., Takai, Y., Borst, J. G., Brose, N., McMahon, H. T., and Verhage, M. (2010). Doc2b Is a High-Affinity Ca2+ Sensor for Spontaneous Neurotransmitter Release. Science 327, 1614-1618.

Han, Y., Kaeser, P. S., Südhof, T. C., and Schneggenburger, R. (2011). RIM Determines Ca2+ Channel Density and Vesicle Docking at the Presynaptic Active Zone. Neuron 69, 304-316.

Harrison, J. M., and Irving, R. (1966). Ascending connections of the anterior ventral cochlear nucleus in the rat. J Comp Neurol 126, 51-63.

Huntwork, S., and Littleton, J. T. (2007). A complexin fusion clamp regulates spontaneous neurotransmitter release and synaptic growth. Nat Neurosci 10, 1235-7.

Ishizuka, T., Saisu, H., Suzuki, T., Kirino, Y., and Abe, T. (1997). Molecular cloning of synaphins/complexins, cytosolic proteins involved in transmitter release, in the electric organ of an electric ray (Narke japonica). Neuroscience Letters 232, 107-110.

Iwasaki, S., Momiyama, A., Uchitel, O. D., and Takahashi, T. (2000). Developmental changes in calcium channel types mediating central synaptic transmission. $J$ Neurosci 20, 59-65.

Iwasaki, S., and Takahashi, T. (2001). Developmental regulation of transmitter release at the calyx of Held in rat auditory brainstem. $J$ Physiol 534, 861-71.

Iyer, J., Wahlmark, C. J., Kuser-Ahnert, G. A., and Kawasaki, F. Molecular mechanisms of COMPLEXIN fusion clamp function in synaptic exocytosis revealed in a new Drosophila mutant. Molecular and Cellular Neuroscience.

Jahn, R., and Fasshauer, D. (2012). Molecular machines governing exocytosis of synaptic vesicles. Nature 490, 201-7. 
Joshi, I., and Wang, L. Y. (2002). Developmental profiles of glutamate receptors and synaptic transmission at a single synapse in the mouse auditory brainstem. $J$ Physiol 540, 861-73.

Kaeser-Woo, Y. J., Yang, X., and Sudhof, T. C. (2012). C-terminal complexin sequence is selectively required for clamping and priming but not for $\mathrm{Ca} 2+$ triggering of synaptic exocytosis. J Neurosci 32, 2877-85.

Katz, B. (1969). THE RELEASE OF NEURAL TRANSMITTER SUBSTANCES. $J$ Neurol Neurosurg Psychiatry 32, 638.

Kochubey, O., Lou, X., and Schneggenburger, R. (2011). Regulation of transmitter release by $\mathrm{Ca} 2+$ and synaptotagmin: insights from a large CNS synapse. Trends in Neurosciences 34, 237-246.

Kochubey, O., and Schneggenburger, R. (2011). Synaptotagmin Increases the Dynamic Range of Synapses by Driving Ca2+-Evoked Release and by Clamping a Near-Linear Remaining Ca2+ Sensor. Neuron 69, 736-748.

Koike-Tani, M., Saitoh, N., and Takahashi, T. (2005). Mechanisms underlying developmental speeding in AMPA-EPSC decay time at the calyx of Held. $J$ Neurosci 25, 199-207.

Kuwabara, N., DiCaprio, R. A., and Zook, J. M. (1991). Afferents to the medial nucleus of the trapezoid body and their collateral projections. J Comp Neurol 314, 684-706.

Lin, K. H., Oleskevich, S., and Taschenberger, H. (2011). Presynaptic Ca2+ influx and vesicle exocytosis at the mouse endbulb of Held: a comparison of two auditory nerve terminals. $J$ Physiol 589, 4301-20.

Lin, M. Y., Rohan, J. G., Cai, H., Reim, K., Ko, C. P., and Chow, R. H. (2013). Complexin facilitates exocytosis and synchronizes vesicle release in two secretory model systems. J Physiol 591, 2463-73.

Lou, X., Scheuss, V., and Schneggenburger, R. (2005). Allosteric modulation of the presynaptic Ca2+ sensor for vesicle fusion. Nature 435, 497-501.

Lu, T., and Trussell, L. O. (2000). Inhibitory Transmission Mediated by Asynchronous Transmitter Release. Neuron 26, 683-694.

Martin, J. A., Hu, Z., Fenz, K. M., Fernandez, J., and Dittman, J. S. (2011). Complexin Has Opposite Effects on Two Modes of Synaptic Vesicle Fusion. Current Biology 21, 97-105.

Maximov, A., and Südhof, T. C. (2005). Autonomous Function of Synaptotagmin 1 in Triggering Synchronous Release Independent of Asynchronous Release. Neuron 48, 547-554.

Maximov, A., Tang, J., Yang, X., Pang, Z. P., and Sudhof, T. C. (2009). Complexin controls the force transfer from SNARE complexes to membranes in fusion. 
Science 323, 516-21.

McMahon, H. T., Missler, M., Li, C., and Südhof, T. C. (1995). Complexins: Cytosolic proteins that regulate SNAP receptor function. Cell 83, 111-119.

Meinrenken, C. J., Borst, J. G., and Sakmann, B. (2002). Calcium secretion coupling at calyx of held governed by nonuniform channel-vesicle topography. $J$ Neurosci 22, 1648-67.

Meiri, U., and Rahamimoff, R. (1972). Neuromuscular transmission: inhibition by manganese ions. Science 176, 308-9.

Miledi, R. (1966). Strontium as a substitute for calcium in the process of transmitter release at the neuromuscular junction. Nature 212, 1233-4.

Naraghi, M., and Neher, E. (1997). Linearized buffered Ca2+ diffusion in microdomains and its implications for calculation of $[\mathrm{Ca} 2+]$ at the mouth of a calcium channel. J Neurosci 17, 6961-73.

Neher, E. (1998). Vesicle Pools and Ca2+ Microdomains: New Tools for Understanding Their Roles in Neurotransmitter Release. Neuron 20, 389-399.

Neher, E. (2010). Complexin: does it deserve its name? Neuron 68, 803-6.

Neher, E., and Sakaba, T. (2008). Multiple Roles of Calcium Ions in the Regulation of Neurotransmitter Release. Neuron 59, 861-872.

Otsu, Y., Shahrezaei, V., Li, B., Raymond, L. A., Delaney, K. R., and Murphy, T. H. (2004). Competition between phasic and asynchronous release for recovered synaptic vesicles at developing hippocampal autaptic synapses. J Neurosci $\mathbf{2 4}$, 420-33.

Pang, Zhiping P., Bacaj, T., Yang, X., Zhou, P., Xu, W., and Südhof, Thomas C. (2011). Doc2 Supports Spontaneous Synaptic Transmission by a Ca2+-Independent Mechanism. Neuron 70, 244-251.

Pang, Z. P., Melicoff, E., Padgett, D., Liu, Y., Teich, A. F., Dickey, B. F., Lin, W., Adachi, R., and Sudhof, T. C. (2006). Synaptotagmin-2 is essential for survival and contributes to $\mathrm{Ca} 2+$ triggering of neurotransmitter release in central and neuromuscular synapses. $J$ Neurosci 26, 13493-504.

Quastel, D. M. (1997). The binomial model in fluctuation analysis of quantal neurotransmitter release. Biophys $J$ 72, 728-53.

Reim, K., Mansour, M., Varoqueaux, F., McMahon, H. T., Südhof, T. C., Brose, N., and Rosenmund, C. (2001). Complexins Regulate a Late Step in Ca2+-Dependent Neurotransmitter Release. Cell 104, 71-81.

Reim, K., Wegmeyer, H., Brandstatter, J. H., Xue, M., Rosenmund, C., Dresbach, T., Hofmann, K., and Brose, N. (2005). Structurally and functionally unique complexins at retinal ribbon synapses. J Cell Biol 169, 669-80.

Rizo, J., and Rosenmund, C. (2008). Synaptic vesicle fusion. Nat Struct Mol Biol 15, 
$665-74$.

Rizzoli, S. O., and Betz, W. J. (2005). Synaptic vesicle pools. Nat Rev Neurosci 6, 57-69.

Rodriguez-Contreras, A., van Hoeve, J. S., Habets, R. L., Locher, H., and Borst, J. G. (2008). Dynamic development of the calyx of Held synapse. Proc Natl Acad Sci U S A 105, 5603-8.

Ryugo, D. K., Wu, M. M., and Pongstaporn, T. (1996). Activity-related features of synapse morphology: a study of endbulbs of held. J Comp Neurol 365, 141-58.

Sabatini, B. L., and Regehr, W. G. (1997). Control of neurotransmitter release by presynaptic waveform at the granule cell to Purkinje cell synapse. J Neurosci 17, 3425-35.

Sakaba, T. (2006). Roles of the fast-releasing and the slowly releasing vesicles in synaptic transmission at the calyx of held. J Neurosci 26, 5863-71.

Sakaba, T., and Neher, E. (2001). Calmodulin Mediates Rapid Recruitment of Fast-Releasing Synaptic Vesicles at a Calyx-Type Synapse. Neuron 32, 1119-1131.

Sara, Y., Virmani, T., Deák, F., Liu, X., and Kavalali, E. T. (2005). An Isolated Pool of Vesicles Recycles at Rest and Drives Spontaneous Neurotransmission. Neuron 45, 563-573.

Satzler, K., Sohl, L. F., Bollmann, J. H., Borst, J. G., Frotscher, M., Sakmann, B., and Lubke, J. H. (2002). Three-dimensional reconstruction of a calyx of Held and its postsynaptic principal neuron in the medial nucleus of the trapezoid body. $J$ Neurosci 22, 10567-79.

Scheuss, V., and Neher, E. (2001). Estimating Synaptic Parameters from Mean, Variance, and Covariance in Trains of Synaptic Responses. Biophysical Journal 81, 1970-1989.

Scheuss, V., Taschenberger, H., and Neher, E. (2007). Kinetics of both synchronous and asynchronous quantal release during trains of action potential-evoked EPSCs at the rat calyx of Held. $J$ Physiol 585, 361-81.

Schneggenburger, R., and Forsythe, I. D. (2006). The calyx of Held. Cell Tissue Res 326, 311-37.

Schneggenburger, R., and Neher, E. (2000). Intracellular calcium dependence of transmitter release rates at a fast central synapse. Nature 406, 889-93.

Schneggenburger, R., Sakaba, T., and Neher, E. (2002). Vesicle pools and short-term synaptic depression: lessons from a large synapse. Trends in Neurosciences $\mathbf{2 5}$, 206-212.

Söllner, T., Bennett, M. K., Whiteheart, S. W., Scheller, R. H., and Rothman, J. E. (1993). A protein assembly-disassembly pathway in vitro that may correspond 
to sequential steps of synaptic vesicle docking, activation, and fusion. Cell 75, 409-418.

Sorensen, J. B. (2009). Conflicting views on the membrane fusion machinery and the fusion pore. Annu Rev Cell Dev Biol 25, 513-37.

Strenzke, N., Chanda, S., Kopp-Scheinpflug, C., Khimich, D., Reim, K., Bulankina, A. V., Neef, A., Wolf, F., Brose, N., Xu-Friedman, M. A., and Moser, T. (2009). Complexin-I is required for high-fidelity transmission at the endbulb of Held auditory synapse. $J$ Neurosci 29, 7991-8004.

Sudhof, T. C. (2002). Synaptotagmins: why so many? J Biol Chem 277, 7629-32.

Sudhof, T. C., and Rothman, J. E. (2009). Membrane fusion: grappling with SNARE and SM proteins. Science 323, 474-7.

Sun, J.-Y., and Wu, L.-G. (2001). Fast Kinetics of Exocytosis Revealed by Simultaneous Measurements of Presynaptic Capacitance and Postsynaptic Currents at a Central Synapse. Neuron 30, 171-182.

Sun, J., Pang, Z. P., Qin, D., Fahim, A. T., Adachi, R., and Sudhof, T. C. (2007). A dual-Ca2+-sensor model for neurotransmitter release in a central synapse. Nature 450, 676-82.

Tang, J., Maximov, A., Shin, O.-H., Dai, H., Rizo, J., and Südhof, T. C. (2006). A Complexin/Synaptotagmin 1 Switch Controls Fast Synaptic Vesicle Exocytosis. Cell 126, 1175-1187.

Taschenberger, H., Leao, R. M., Rowland, K. C., Spirou, G. A., and von Gersdorff, H. (2002). Optimizing synaptic architecture and efficiency for high-frequency transmission. Neuron 36, 1127-43.

Taschenberger, H., Scheuss, V., and Neher, E. (2005). Release kinetics, quantal parameters and their modulation during short-term depression at a developing synapse in the rat CNS. $J$ Physiol 568, 513-37.

Taschenberger, H., and von Gersdorff, H. (2000). Fine-tuning an auditory synapse for speed and fidelity: developmental changes in presynaptic waveform, EPSC kinetics, and synaptic plasticity. $J$ Neurosci 20, 9162-73.

Trussell, L. O. (1999). Synaptic mechanisms for coding timing in auditory neurons. Annu Rev Physiol 61, 477-96.

von Gersdorff, H., and Borst, J. G. (2002). Short-term plasticity at the calyx of held. Nat Rev Neurosci 3, 53-64.

von Gersdorff, H., Schneggenburger, R., Weis, S., and Neher, E. (1997). Presynaptic depression at a calyx synapse: the small contribution of metabotropic glutamate receptors. J Neurosci 17, 8137-46.

Wang, L. Y., Neher, E., and Taschenberger, H. (2008). Synaptic vesicles in mature calyx of Held synapses sense higher nanodomain calcium concentrations 
during action potential-evoked glutamate release. J Neurosci 28, 14450-8.

Weis, S., Schneggenburger, R., and Neher, E. (1999). Properties of a model of $\mathrm{Ca}++$-dependent vesicle pool dynamics and short term synaptic depression. Biophys J 77, 2418-29.

Wu, X. S., Xue, L., Mohan, R., Paradiso, K., Gillis, K. D., and Wu, L. G. (2007). The origin of quantal size variation: vesicular glutamate concentration plays a significant role. J Neurosci 27, 3046-56.

Xiao, L., Han, Y., Runne, H., Murray, H., Kochubey, O., Luthi-Carter, R., and Schneggenburger, R. (2010). Developmental expression of Synaptotagmin isoforms in single calyx of Held-generating neurons. Molecular and Cellular Neuroscience 44, 374-385.

Xue, M., Lin, Y. Q., Pan, H., Reim, K., Deng, H., Bellen, H. J., and Rosenmund, C. (2009). Tilting the balance between facilitatory and inhibitory functions of mammalian and Drosophila Complexins orchestrates synaptic vesicle exocytosis. Neuron 64, 367-80.

Xue, M., Reim, K., Chen, X., Chao, H. T., Deng, H., Rizo, J., Brose, N., and Rosenmund, C. (2007). Distinct domains of complexin I differentially regulate neurotransmitter release. Nat Struct Mol Biol 14, 949-58.

Yamashita, T., Hige, T., and Takahashi, T. (2005). Vesicle endocytosis requires dynamin-dependent GTP hydrolysis at a fast CNS synapse. Science 307, 124-7.

Yang, X., Kaeser-Woo, Y. J., Pang, Z. P., Xu, W., and Sudhof, T. C. (2010a). Complexin clamps asynchronous release by blocking a secondary $\mathrm{Ca}(2+)$ sensor via its accessory alpha helix. Neuron 68, 907-20.

Yang, Y. M., Fedchyshyn, M. J., Grande, G., Aitoubah, J., Tsang, C. W., Xie, H., Ackerley, C. A., Trimble, W. S., and Wang, L. Y. (2010b). Septins regulate developmental switching from microdomain to nanodomain coupling of $\mathrm{Ca}(2+)$ influx to neurotransmitter release at a central synapse. Neuron $\mathbf{6 7}$, $100-15$.

Yang, Y. M., and Wang, L. Y. (2006). Amplitude and kinetics of action potential-evoked $\mathrm{Ca} 2+$ current and its efficacy in triggering transmitter release at the developing calyx of held synapse. J Neurosci 26, 5698-708.

Yao, J., Gaffaney, Jon D., Kwon, Sung E., and Chapman, Edwin R. (2011). Doc2 Is a $\mathrm{Ca} 2+$ Sensor Required for Asynchronous Neurotransmitter Release. Cell 147, 666-677.

Young Jr, S. M., and Neher, E. (2009). Synaptotagmin Has an Essential Function in Synaptic Vesicle Positioning for Synchronous Release in Addition to Its Role as a Calcium Sensor. Neuron 63, 482-496. 
Zucker, R. S., and Regehr, W. G. (2002). Short-term synaptic plasticity. Annu Rev Physiol 64, 355-405. 


\title{
Appendix
}

\author{
Abbreviations \\ aCSF artificial cerebrospinal fluid \\ AMPA a-Amino-3-hydroxy-5-methyl-4-isoxazolepropionic acid \\ AP action potential \\ aVCN anterior ventral cochlear nucleus \\ AZ active zone \\ EGTA Ethylene glycol-bis(2-aminoethylether)-N,N,N',N'-tetraacetic acid \\ EM electron microscopy \\ EPSC excitatory postsynaptic current \\ GBC globular bushy cell \\ KO Knock out \\ mEPSC miniature excitatory postsynaptic current \\ MNTB medial nucleus of the trapezoid body \\ $\mathrm{N}$ the number of releasable vesicles \\ $\mathrm{p} \quad$ release probability \\ q quantal size \\ RRP readily releasable pool \\ RT room temperature \\ SEM standard error of the mean \\ SNAP soluble NSF attachment protein \\ SNARE SNAP receptor \\ Syt Synaptotagmin \\ TEA Tetraethylammonium \\ TTX Tetrodotoxin \\ vGlut Vesicular glutamate transporter \\ UV Ultraviolet \\ WT wild type
}




\section{Acknowledgment}

Foremost, I would like to express my sincere gratitude to Prof. Dr. Erwin Neher for the opportunity to work on my $\mathrm{Ph} . \mathrm{D}$. thesis in his department, and my supervisor Dr. Holger Taschenberger, for his constant encouragement and support throughout my research, for his patience, motivation, enthusiasm and solid knowledge. I can not imagine to have a better advisor and mentor for my Ph.D. study.

I am grateful to the head of my Ph.D. program (Sensory and Motor Neuroscience program of Goettingen University, Germany), Prof. Dr. Tobias Moser, who supported my application to the program and was one of the members of my Ph.D. committee. Without his support, my Ph.D. study would not have started. I would also like to thank the second member of my Ph.D. committee, Dr. Oliver Schlüter, for giving me valuable advice during the committee meetings. I would like to acknowledge Dr. Kerstin Reim, for her generosity to provide CPXI knock-out mice, and performed the western blot analysis for this thesis. Also, Dr. Meike Pedersen, whom I am indebted to, for taking the beautiful immunofluorescence images for this project. I would also like to thank my colleague, Dr. Kun-Han Lin for his numerous helpful suggestions during my $\mathrm{PhD}$ study. Also, it was my pleasure to be able to work with Prof. Dr. Takeshi Sakaba, Dr. Chao-Hua Huang, Dr. Yunfeng Hua, Dr. Andrew Woehler, Dr. Lijun Yao, Dr. Raunak Sinha, and other colleagues who helped me in one way or another: Ms. Ina Herfort, Ms. Irmgard Barteczko, Ms. Sigrid Schmidt, Mr. Frank Würriehausen, Mr. Frank Köhne, Mr. Dirk Reuter. I thank all my friends, especially to Jian-Hua Chen, Shih-Ju Lee, He-Hsuan Hsiao, Yen-Ying Chen, Tzu-Lun Wang, and Mei-Chih Liao. Without these friends in Göttingen, I wouldn't believe I could have started a life in a foreign country. The critical comments and suggestions from the examiners are very much appreciated. I dedicate this thesis to my father and my 
mother whom I owe all that I have become today. I thank my sister and brother and Leo E Wong for their love and support. 
Personal Data

Name

Shuwen Chang

Gendor

Female

Date of birth

30.06.1983

Place of birth

Kaohsiung, Taiwan

Present Citizenship

Taiwan

\section{Education}

\section{August 2010 PHD STUDENT}

Present Max Planck Institute for Biophysical Chemistry, Goettingen,

Germany

Sensory and Motor Neuroscience program in the Göttingen graduate school for neurosciences and molecular biosciences (GGNB)

Georg August-University Göttingen, Germany

July 2007 MASTER OF SCIENCE

Major: Biochemistry and Molecular Biology

Topic: The role of CCAAT/Enhancer binding protein alpha in the expression of DDR2

National Yang-Ming University, Taipei, Taiwan

June 2005 BACCALAUREATE OF SCIENCE

Major: Biochemistry and Molecular Biology

National Cheng-Kung University, Tainan, Taiwan 
Hinrich Kielblock, Shuwen Chang, Birk Urmersbach, Holger Taschenberger, Marc Timme, When Less Is More: Non-monotonic Spike Sequence Processing in Neurons Caused by Graded Loss of Resources, PLOS Computational Biology, Submitted.

Holger Taschenberger, Kun-Han Lin, Shuwen Chang Presynaptic Ca2+ Influx and Its Modulation at Auditory Calyceal Terminals in Modulation of Presynaptic Calcium Channels, Springer Publishing (2013) 